\title{
Representação e Quantificação de \\ Redes Vasculares a Partir de Imagens de Angiografia Tridimensional
}

\author{
Miguel Angel Galarreta Valverde
}

TESE APRESENTADA

AO

Instituto de MATEMÁtiCA E EstatísticA

DA

UNIVERSIDADE DE SÃO PAULO

PARA

OBTENÇÃO DO TÍTULO

$\mathrm{DE}$

DOUTOR EM CIÊNCIAS

Programa: Ciência da Computação

Orientador: Prof. Dr. Marcel Parolin Jackowski

Durante o desenvolvimento deste trabalho o autor recebeu auxílio financeiro da CAPES

São Paulo, Janeiro de 2018 


\title{
Representação e Quantificação de Redes Vasculares a Partir de Imagens de Angiografia Tridimensional
}

\author{
Esta tese contém as correções e alterações \\ sugeridas pela Comissão Julgadora durante a defesa \\ realizada por Miguel Angel Galarreta Valverde em 12/12/2017. \\ O original encontra-se disponível no Instituto de \\ Matemática e Estatística da Universidade de São Paulo.
}

Banca Examinadora:

- Prof. Dr. Marcel Parolin Jackowski (orientador) - IME-USP

- Prof. Dr. Fatima Lourdes Santos Nunes - EACH-USP

- Prof. Dr. Paulo Andre Vechiatto de Miranda - IME-USP

- Prof. Dr. Ivan Hong Jun Ker - FM-USP

- Prof. Dr. Ronaldo Fumio Hashimoto - IME-USP 


\section{Agradecimentos}

Agradeço a meu orientador, o prof. Marcel P. Jackowski, por ter me guiado ao longo do meu mestrado e do meu doutorado.

Aos professores membros da banca examinadora por seus inestimáveis aportes. À prof. Fatima e ao prof. Choukri por seus comentários e sugestões.

A todos meus amigos, tanto os que fizeram mais aprazível minha estadia neste belo pais, como os que moram em Peru, aos quais senti sempre presentes.

Em especial agradeço à minha família, que apesar da distância estiveram apoiando-me em cada momento. Agradeço muito especialmente também à minha noiva Mariela pelo carinho e por me acompanhar nesta jornada. Este trabalho não teria sido possível sem eles.

Finalmente, agradeço à agencia de financiamento CAPES por ter me concedido a bolsa de doutorado. 


\section{Resumo}

VALVERDE, MIGUEL A. G. Representação e Quantificação de Redes Vasculares a Partir de Imagens de Angiografia Tridimensional. 2018. 94 f. Tese (Doutorado) - Instituto de Matemática e Estatística, Universidade de São Paulo, São Paulo, 2018.

As imagens de Angiografia por Ressonância Magnética (angio-RM) e Tomografia Computadorizada (angio-TC) são ferramentas amplamente usadas em processos de quantificação vascular e no diagnóstico de doenças cardiovasculares, as quais são consideradas entre as principais causas de morte. Contudo, a análise dos vasos em larga escala a partir das imagens é dificultada, tanto pela variabilidade natural dos vasos no corpo humano, quanto pela grande quantidade de dados disponíveis. Além disso, os métodos de quantificação existentes, usualmente extraem as características a partir dos esqueletos, ou até mesmo das próprias imagens de angiografia, razão pela qual tais métodos podem fazer necessária a reanálise das imagens repetidas vezes.

Com o intuito de facilitar a análise e de fornecer uma ferramenta de apoio ao diagnóstico, neste trabalho são apresentados um modelo de representação textual de redes vasculares e uma metodologia de quantificação vascular automática, que é feita a partir dessa representação. A representação é obtida a partir da segmentação de imagens volumétricas de angio-RM e angio-TC, seguida da extração de trajetórias e diâmetros de redes vasculares. Tal representação é híbrida, combinando grafos e uma sequência textual de instruções, e permite não apenas a extração de caraterísticas morfológicas da rede vascular, como também a compressão das imagens e, ainda, a reconstrução de imagens similares às imagens originais.

A partir das características extraídas, foram realizados estudos comparativos entre arquiteturas vasculares, o que é feito tanto por meio do uso de imagens sintéticas, como por meio de imagens reais, imagens nas quais foi possível encontrar diferenças entre arquiteturas, além de viabilizar a caracterização de aneurismas em um indivíduo. Paralelamente, desenvolvemos um método que permite identificar similaridade entre segmentos vasculares, o que por sua vez possibilita o reconhecimento e rotulação de segmentos em um conjunto de redes vasculares. 
A metodologia por nós desenvolvida deve também auxiliar no desenvolvimento de processos de classificação de vasos sanguíneos, de ferramentas para o diagnóstico automático de doenças vasculares, e para a melhora de técnicas utilizadas na prática clínica.

Palavras-chave: angiografia, quantificação, caracterização, morfologia vascular. 


\section{Abstract}

Magnetic Resonance Angiography (angio-MR) and Computed Tomography Angiography (angio-TC) are widely used imaging techniques used for vascular quantification and the diagnosis of cardiovascular diseases, considered one of the main causes of death. Blood vessel analysis using angiographic images is intrisically difficult because of the natural human vessel variability and the large amount of information. Additionally, most quantification methods perform the analysis of entire datasets, which can be very time consuming when they need to be reanalyzed.

With the aim of reducing these problems and to provide a tool to aid diagnosis, we propose a textual representation model for vascular networks and an automatic vascular quantification methodology. The representation is obtained from volumetric image segmentation of angio-MR and angio-TC, followed by the extraction of the trajectory and the blood vessel diameters. This representation is hybrid in nature, combining graphs and a sequence of textual instructions, allowing for the extraction of morphological features, image compression, and the synthesis of angiographic images.

Using extracted features derived from the model, comparative studies of vascular architecture can be performed. Experiments were made using synthetic and real images, in which was possible to find structural differences that make feasible to characterize abnormalities such as aneurysms. Also, a vessel similarity identification method was developed, which makes it possible to recognize vessel segments and label them in a set of vessel networks. The proposed methodology should aid in blood vessel classification processes, automatic diagnosis of cardiovascular diseases, and allow development of methods and applications that could be used in the clinical practice.

Keywords: angiography, quantification, characterization, vascular morphology. 


\section{Sumário}

Siglas $\quad$ xiii

Lista de Figuras $\quad$ xv

$\begin{array}{ll}\text { Lista de Tabelas } & \text { xvii }\end{array}$

1 Introdução 1

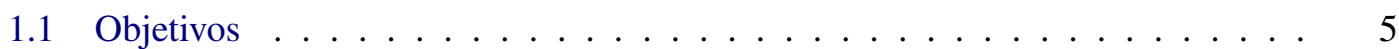

1.1.1 Objetivos específicos .................. 5

1.2 Aplicações . . . . . . . . . . . . . . . . . . . . . . 6

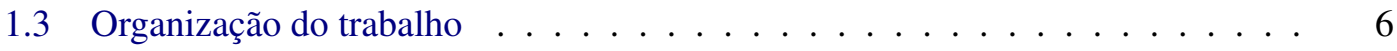

2 Trabalhos correlatos $\quad 7$

2.1 Planejamento da revisão $\ldots \ldots \ldots \ldots$. . . . . . . . . . . . 7

2.1 .1 Método de busca . . . . . . . . . . . . . . . . . 8

2.2 Realização da revisão . . . . . . . . . . . . . . . . . . . . . . 9

2.2.1 Seleção de artigos . . . . . . . . . . . . . . . . . . 9

2.3 Revisão . . . . . . . . . . . . . . . . . . . . . . . . . . 11

3 Conceitos preliminares 17

3.1 Imagens médicas . . . . . . . . . . . . . . . . . . . . . . . 17

3.1.1 Coordenadas do voxel e do objeto . . . . . . . . . . . . . . 17

3.2 Segmentação . . . . . . . . . . . . . . . . . . . . . 18

3.2 .1 Vesselness . . . . . . . . . . . . . . . . . . . 18

3.3 Esqueletização . . . . . . . . . . . . . . . . . . . 20

3.4 Redes vasculares sintéticas . . . . . . . . . . . . . . . . . . . . . . . 20

3.4.1 Sistemas de Lindenmayer . . . . . . . . . . . . . . . . . 20

3.4.2 Geração de imagens sintéticas com L-systems estocásticos . . . . . . . 21 
3.4.2.1 Variáveis iniciais . . . . . . . . . . . . . . . 21

3.4.2.2 Caracteres permitidos na representação . . . . . . . . . 22

3.4.3 Geração de vasos sintéticos com L-systems . . . . . . . . . . . . . 22

3.4.4 Limitação dos L-systems estocásticos na geração de vasos sintéticos . . 23

3.5 Teoria de Grafos . . . . . . . . . . . . . . . . . . . 23

3.5 .1 Definições básicas . . . . . . . . . . . . . . . . 23

3.6 Quantificação . . . . . . . . . . . . . . . . . . . . . . . 24

3.6.1 Características na literatura . . . . . . . . . . . . . . . 24

3.6.1.1 Raios e comprimentos . . . . . . . . . . . . . 25

3.6.1.2 Tortuosidade . . . . . . . . . . . . . . . 25

3.6.1.3 Características topológicas _............ 28

4 Metodologia 2

4.1 Pré-processamento . . . . . . . . . . . . . . . . . . . . . . . 29

4.1 .1 Segmentação . . . . . . . . . . . . . . . . . . . . . . . . 29

4.2 Extração da Representação . . . . . . . . . . . . . . . . . . . . . . . . 31

4.2 .1 Esqueletização . . . . . . . . . . . . . . . . . . . . 32

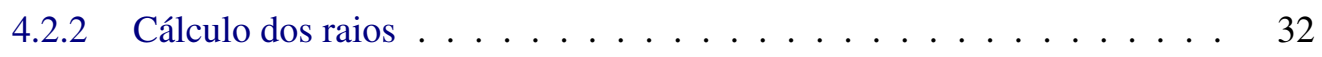

4.2 .3 Processamento do esqueleto . . . . . . . . . . . . . . 33

4.2.3.1 Construção do grafo para processamento do esqueleto . . . . 33

4.2.3.2 Atribuição de identificadores de segmento . . . . . . . . . . 34

4.2.3.3 Resolução das regiões de conflito . . . . . . . . . . . . . . . . . . . 37

4.2.3.4 Remoção de falsos segmentos . . . . . . . . . . . . . . . . 37

4.2.4 Determinação de pontos de controle . . . . . . . . . . . . . . . . . . . 38

4.2.5 Geração da representação em cadeia de texto . . . . . . . . . . . . . 43

4.2 .6 Representação de um segmento . . . . . . . . . . . . . . . . . . . . . . . . 44

4.2 .7 Estrutura da rede vascular . . . . . . . . . . . . . . . . . . . 47

4.2 .8 Armazenamento . . . . . . . . . . . . . . . . . . 49

4.2 .9 Reconstrução . . . . . . . . . . . . . . . . . . . . . . . . . . . . 49

4.3 Quantificação . . . . . . . . . . . . . . . . . . . . 49

4.3 .1 Construção do grafo de representação . . . . . . . . . . . . . . . 50

4.3.2 Extração de características . . . . . . . . . . . . . . . . . . . . 51

4.3.2.1 Quantidade de elementos . . . . . . . . . . . 52

4.3.2.2 Comprimento, raio e outros atributos dos segmentos . . . . 52

4.3.2.3 Métrica de distância (Distance Metric, DM) . . . . . . . . 53 
4.3.2.4 Métrica de contagem de pontos de inflexão (Inflection Counting Metric, ICM) . . . . . . . . . . . . 53

4.3.2.5 Métrica de soma de ângulos (Sum Of Angles Metric, SOAM) 54

4.3.2.6 Ordem de Strahler . . . . . . . . . . . . . . . 54

4.3.2.7 Taxonomia de Tokunaga . . . . . . . . . . . . 55

4.4 Aplicações .............................. 56

4.4.1 Segmentação interativa . . . . . . . . . . . . . 56

4.4.2 Correspondência entre redes vasculares . . . . . . . . . . . 57

5 Resultados $\quad 61$

5.1 Conjunto de imagens ......................... 61

5.1.1 Dataset de imagens sintéticas ............... 61

5.1 .2 Dataset de imagens Reais . . . . . . . . . . . . . 64

5.1.2.1 Imagens de angiografia da artéria carótida interna . . . . . 64

5.1.2.2 Imagens reconstruídas a partir do projeto Brava . . . . . . 65

5.2 Avaliação do processo de reconstrução de redes vasculares . . . . . . . . . . 66

5.3 Quantificação a partir de imagens sintéticas . . . . . . . . . . . 70

5.3.1 Quantificação com a taxonomia de Tokunaga . . . . . . . . . 72

5.3.2 Alinhamento de sequências . . . . . . . . . . . . . 73

5.4 Quantificação a partir de imagens reais . . . . . . . . . . . . . . . 74

5.5 Correspondência entre redes vasculares . . . . . . . . . . . . 75

6 Conclusões $\quad 83$

6.1 Síntese do trabalho ........................ 83

6.2 Contribuições ............................ 84

6.3 Limitações . . . . . . . . . . . . . . . . . . . 85

6.4 Trabalhos futuros ....................... 85

$\begin{array}{lr}\text { Referências Bibliográficas } & \mathbf{8 7}\end{array}$ 


\section{Siglas}

ACA artéria cerebral anterior. 77, 79, 80

ACI artéria carótida interna. 77, 79

ACM artéria cerebral média. 77

ACoP artéria comunicante posterior. 77, 79, 81

angio-RM Angiografia por Ressonância Magnética. 1, 17, 49, 66, 83, 85

angio-TC Angiografia por Tomografia Computadorizada. 1, 17, 49, 66, 83, 85

CoW Circle of Willis (círculo de Willis). 47, 50, 75-77

DM Distance Metric (métrica de distância). 25, 26, 51, 53, 58

ICM Inflection Counting Metric (métrica de contagem de pontos de inflexão). 25, 26, 51, 53, 58

MST Minimum Spanning Tree (árvore de espalhamento mínima). 54, 55

NRMSE Normalized Root Mean Squared Error (raiz do erro quadrático médio normalizado). $67-69$

SOAM Sum Of Angles Metric (métrica de soma de ângulos). 25, 26, 51, 54, 58, 72

SSIM Structural Similarity (similaridade estrutural). 68, 69 


\section{Lista de Figuras}

1.1 Exemplos de imagens de angiografia . . . . . . . . . . . . . 2

1.2 Diagrama de funcionamento da metodologia proposta. . . . . . . . . . . . 5

2.1 Fluxo da análise de artigos $\ldots \ldots \ldots \ldots$

2.2 Características mais usadas . . . . . . . . . . . . . . . . . 15

3.1 coordenadas do objeto e coordenadas da imagem f . . . . . . . . . . . . . 18

3.2 Principais direções e os autovalores usados pelo método de vesselness . . . . . 19

3.3 Triedro de Serret-Frennet. . . . . . . . . . . . . . . . . . . . . . . . 27

4.1 Fluxo representativo da extração de sequências a partir de imagens de angiografia 3D . . . . . . . . . . . . . . . . . . . 31

4.2 Exemplo de esqueletização de uma imagem 3D . . . . . . . . . . . . . . . 32

4.3 Exemplo de grafo obtido a partir de um esqueleto . . . . . . . . . . . . . . . . . . . . . . . . . 35

4.4 Exemplos de grafos obtidos a partir de esqueletos . . . . . . . . . . . . . . . 36

4.5 Atribuição de identificadores de segmento . . . . . . . . . . . . . 36

4.6 Exemplos de resolução de conflitos do grafo na Figura 4.4b. . . . . . . . . . . 39

4.7 Exemplo de pontos de controle equidistantes que não representam a curva adequadamente. . . . . . . . . . . . . . . . . . . . . . 39

4.8 Simulação do algoritmo de escolha de pontos significativos . . . . . . . . . . . 41

4.9 Pontos significativos que não caracterizam adequadamente uma curva . . . . . 42

4.10 Extração da representação de um segmento . . . . . . . . . . . . . . . . 46

4.11 Representação de uma rede vascular em forma de árvore . . . . . . . . . . . . 48

4.12 Representação de uma estrutura cíclica . . . . . . . . . . . . . . . . . . . . . . . . . 48

4.13 Formato de armazenamento da cadeia de representação . . . . . . . . . . . . . 49

4.14 Grafo representativo de uma estrutura vascular . . . . . . . . . . . . . . 51

4.15 Dados usados para o cálculo da DM. . . . . . . . . . . . . . . . . . 53

4.16 Valores de Strahler obtidos a partir de um grafo . . . . . . . . . . . . 55 
4.17 Valores de Tokunaga correspondente ao grafo mostrado na Figura 4.14 . . . . 56

4.18 Matriz de Tokunaga e sua representação com intensidades . . . . . . . . . . 56

5.2 Imagens sintéticas de angiografia com padrão de ramificação regular. . . . . . . 62

5.1 Imagens sintéticas de angiografia composta por segmentos retos. . . . . . . . 63

5.3 Imagens sintéticas de angiografia com padrão e bifurcação não regular. . . . . . 63

5.4 Artéria carótida interna e artéria média direita de dois indivíduos, reconstruídas 64

5.5 Imagem do projeto Brava e a reconstrução de suas artérias . . . . . . . . . . . 65

5.6 Reconstruções com diferentes erros permitidos em vista axial . . . . . . . . . . 66

5.7 Reconstruções com diferentes erros permitidos em vista coronal . . . . . . . . . . . 67

5.8 Avaliação do processo de reconstrução . . . . . . . . . . . . . . . . . . . 68

5.9 Comparação entre a imagem base e suas reconstruções com diferentes valores de erro permitido. . . . . . . . . . . . . . . . . . . . 69

5.10 Quantidade de caracteres da representação a diferentes níveis de detalhe . . . . 70

5.11 Imagens sintéticas de angiografia com ramificações regulares . . . . . . . . . 72

5.12 Imagens sintéticas de angiografia com padrões de ramificação irregular . . . . . 73

5.13 Segmentação do Círculo de Willis usada como base de comparação no experimento de comparação . . . . . . . . . . . . . . . . . . . 76

5.14 Eixos de orientação . . . . . . . . . . . . . . . . . . . . . . . . 77

5.15 Resultados de correspondência com todos os segmentos detectados . . . . . . . 78

5.16 Segmentos da Artéria carótida interna (ACI) corretamente detectado . . . . . . 79

5.17 Segmentos da Artéria cerebral anterior (ACA) corretamente detectado . . . . . 80

5.18 Segmentos da Artéria comunicante posterior (ACoP) corretamente detectado . . 81

5.19 Segmentos da artéria Basilar corretamente detectado . . . . . . . . . . 82 


\section{Lista de Tabelas}

2.1 Cadeias de busca usadas nos diferentes repositórios . . . . . . . . . . . . . . 9

2.2 Artigos resultantes da revisão sistemática (parte 1) . . . . . . . . . . . . . . . 12

2.3 Artigos resultantes da revisão sistemática (parte 2). . . . . . . . . . . . . . . . . . 13

5.1 Características obtidas a partir do conjunto das imagens de teste. . . . . . . . . . . 71

5.2 Comparação entre arquiteturas vasculares . . . . . . . . . . . . . . . . . 74

5.3 Resultados de alinhamento das sequências dos vasos . . . . . . . . . . . . . . 74

5.4 Características obtidas a partir do conjunto de imagens de exemplo. . . . . . . . . . . 75

5.5 Precisão das diferentes artérias do Círculo de Willis (Circle of Willis, CoW). . . 77 


\section{Capítulo 1}

\section{Introdução}

As doenças cardiovasculares são consideradas uma das principais causas de morte em homens e mulheres no Brasil, sendo que em 2009 foram a causa de cerca de $20 \%$ de todas as mortes em indivíduos acima de 30 anos [Mansur e Favarato, 2012]. O problema também é recorrente em países desenvolvidos como os Estados Unidos [Centers for Disease Control and Prevention, 2015], onde a taxa de mortalidade causada por doenças cardiovasculares atingiu $30,67 \%$ do total de mortes no ano 2013. Em escala global, as doenças cardiovasculares foram as principais causas de morte no ano 2012 [Organization et al., 2014]. Dados como estes mostram a grande importância do diagnóstico e tratamento de doenças cardiovasculares. Por tal motivo é necessário prover, ao médico especialista, ferramentas que o auxiliem nesse processo.

Atualmente, entre as principais ferramentas para o diagnóstico figuram as imagens de Angiografia por Tomografia Computadorizada (angio-TC) e de Angiografia por Ressonância Magnética (angio-RM). As imagens de angio-TC são adquiridas por uma técnica de emissão de raios-X. Esses raios são parcialmente absorvidos e refletidos pelos tecidos do corpo, o que depende da composição do tecido. Trata-se de uma radiação ionizante que é especialmente absorvida pelos ossos, os quais adquirem uma tonalidade mais clara nas imagens. $\mathrm{O}$ uso desse método para a aquisição de imagens de vasos sanguíneos requer um contraste endovenoso que possibilite realçá-los na imagem, já que o sangue não absorve esse tipo de radiação [Suetens, 2009].

As imagens por ressonância magnética por sua vez são obtidas por uma técnica de imageamento que não usa radiação ionizante. Nessa técnica, os tecidos são submetidos a um grande campo magnético, que impõe um alinhamento dos prótons dos tecidos com este campo. Logo após, são aplicadas pequenas perturbações no campo magnético que desalinham os prótons. Estes, ao voltar a seu estado de equilíbrio devolvem energia, a qual é mensurada e usada para 
produzir a imagem de RM [Suetens, 2009]. Na Figura 1.1 são mostrados cortes de imagens de angio-TC, angio-RM e uma projeção de máxima intensidade, respectivamente.

Figura 1.1: Exemplos de imagens de angiografia. a) corte axial de imagem por Angio-TC do tórax. b) Corte axial de imagem de Angio-RM do cérebro. c) Projeção de máxima intensidade da imagem de RM.
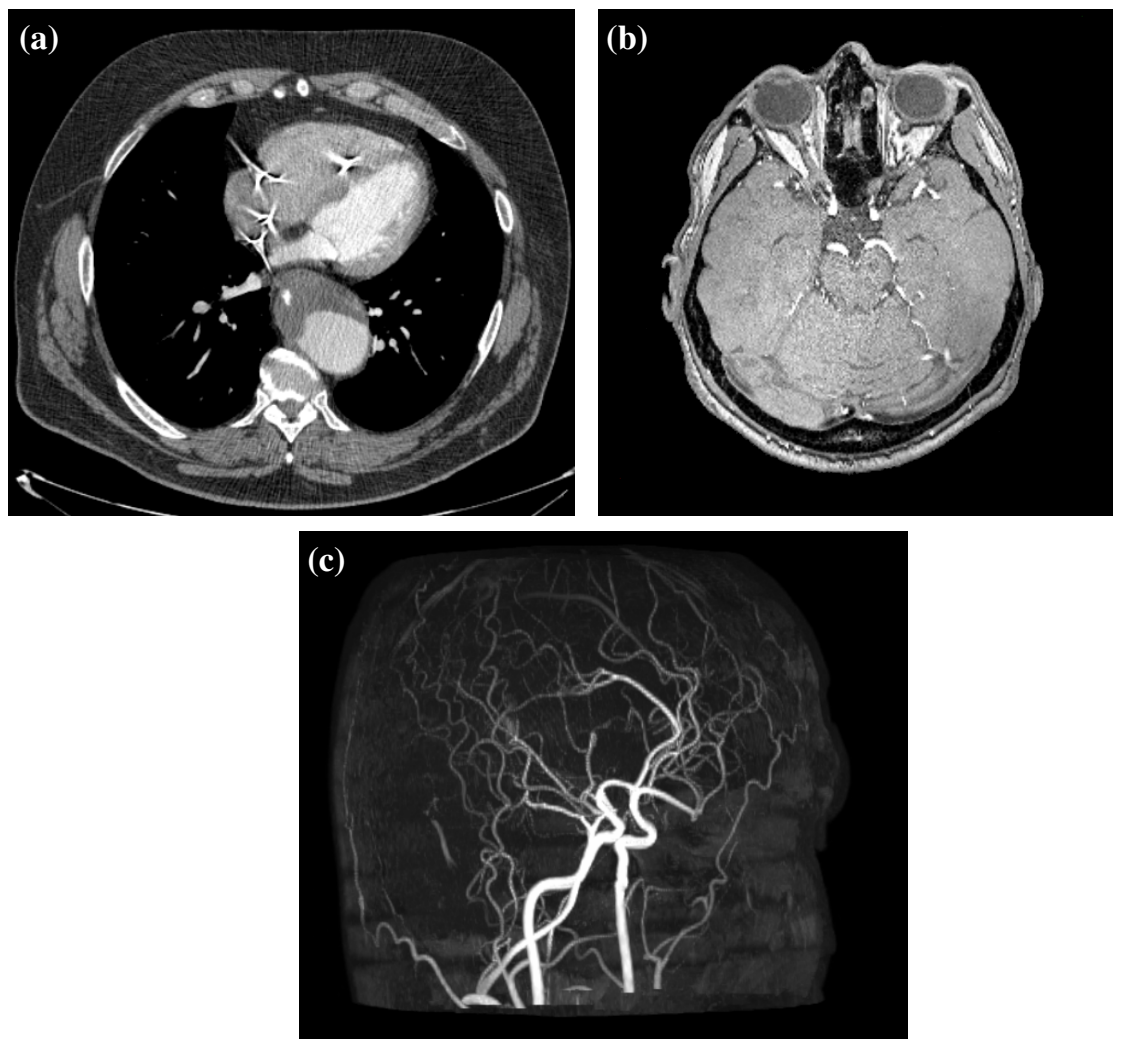

O processo de diagnóstico é dificultado por alguns fatores, como a variabilidade dos vasos nas diferentes partes do corpo e o grande volume de imagens de angiografia [Kocher et al., 2011], o que torna necessário um aprimoramento dos métodos de diagnóstico assistido por computador, de modo que estes possam auxiliar na avaliação das redes vasculares de forma consistente.

O processo de quantificação de estruturas ramificadas em 2D tem sido realizado a partir de diferentes tipos de imagens, tais como as imagens de retina [Perez et al., 2002], ou de membranas corioalantóicas de embrião de galinha [De-La-Cruz, 2015]. Em relação às imagens angiográficas 3D, existem métodos como o proposto por Kang et al. [2009], que foca na análise das deformações das linhas centrais dos vasos; ou o método de Versteylen et al. [2013], que calcula a área do lúmen dos vasos para detectar a presença de cálcio obstruindo os vasos. Porém, o uso de imagens 3D aumenta não só a complexidade da detecção dos vasos como também a da extra- 
ção de suas características, uma vez que seu processamento requer métodos que considerem sua natureza tridimensional e, consequentemente, requer-se maior capacidade de armazenamento e processamento.

Um processo prévio que é essencial à quantificação de imagens de angiografia é a segmentação dos vasos, na qual as redes vasculares são separadas do fundo da imagem, podendo ser este fundo formado por ossos e/ou outros tecidos. A literatura é rica em trabalhos de segmentação de vasos, contudo continua sendo considerado um dos principais tópicos nas pesquisas realizadas nos últimos anos [Lesage et al., 2009]. A segmentação no espaço tridimensional pode ser abordada por diferentes técnicas, tais como as baseadas em cortes em grafos [Zhu e Chung, 2013], as que se baseiam em superfícies de nível (level sets) [Hong e Wang, 2013; Van Bemmel et al., 2004], ou ainda as focadas em melhorar segmentações iniciais feitas manualmente [Nowinski et al., 2009].

O principal foco do presente trabalho é a modelagem, caracterização e quantificação de vasos sanguíneos, os quais são passos necessários para o desenvolvimento de processos mais complexos como o diagnóstico automático e a assistência no planejamento e execução de cirurgias [Passat et al., 2006]. Esse processo pode ser feito a partir de imagens angiográficas 2D ou $3 \mathrm{D}$, para as quais se requer a extração de diversas características.

Diversos trabalhos escolhem a estrutura de árvores como uma forma de representar a organização dos vasos. Tal escolha deve-se principalmente à natureza ramificada dos vasos, que sugere este tipo de representação. No entanto, também se constatam ciclos nas redes vasculares, os quais se observam tanto em capilares sanguíneos, quanto em junções de vasos (anastomoses), ou ainda em estruturas específicas como o círculo de Willis [Alastruey et al., 2007]. A existência de ciclos sugere a necessidade de usar grafos genéricos que permitam incorporar esses ciclos na representação das redes vasculares.

A representação de árvores vasculares como cadeias de texto também é encontrada na literatura. Megalooikonomou et al. [2009], por exemplo, mostra uma metodologia desenvolvida para analisar padrões de bifurcação. $\mathrm{O}$ autor atribui um identificador a cada segmento, e através de uma varredura em profundidade, uma cadeia de texto composta por estes identificadores é obtida. Esta cadeia é utilizada posteriormente na comparação de estruturas vasculares. No entanto, essa metodologia é focada apenas na caracterização de bifurcações. Posteriormente, Valverde [2012] apresenta uma metodologia de geração de redes vasculares sintéticas a partir de gramáticas de Lindenmayer. Estas gramáticas geram uma cadeia de texto intermediária que contém instruções; por meio destas instruções geram-se as redes vasculares sintéticas. Porém, uma vez que nesta metodologia utilizam-se estruturas do tipo árvore, a presença de ciclos não está con- 
templada. Ademais, trata-se de uma metodologia na qual não foram estabelecidos processos para a quantificação dos vasos sanguíneos a partir de suas representações.

Um dos objetivos deste trabalho é possibilitar a representação de ciclos de redes vasculares, por meio do uso de cadeias de instruções em forma de texto e grafos, de maneira que estes possam ser usados para o armazenamento e posterior análise dos vasos. Para tanto, serão extraídas informações dos vasos a partir da sua representação em grafos e cadeias de texto. A representação textual com o intuito de viabilizar o processamento tanto de estruturas do tipo árvore, quanto de estruturas vasculares contendo ciclos, e que permite sua posterior análise é inédita.

O passo seguinte à representação é a extração de características. Métodos de caracterização de imagens 2D, tais como [De-La-Cruz, 2015], que analisa membranas corioalantóicas de embrião de galinha, sugere o uso de algumas métricas baseadas em densidades, estruturas e histogramas. Já [Perez et al., 2002] estuda a quantificação de propriedades geométricas e topológicas da vasculatura na retina. Esse processo é feito usando imagens 2D resultantes de um processo chamado angiografia com fluorescência. Nas imagens 3D, várias características podem ser extraídas. Por exemplo, Kang et al. [2009] foca-se na análise das deformações do esqueleto dos vasos; Bullitt et al. [2003] calcula a tortuosidade de artérias cerebrais; [Valverde et al., 2015] faz uso de características topológicas; [Van Bemmel et al., 2004] usa a área do lúmen, e [Sun e Cao, 2011] extrai os ângulos de bifurcação.

Na prática, já existem aplicativos comerciais que permitem realizar a análise e quantificação de redes vasculares. Alguns deles permitem definir manualmente posições dos vasos, nas quais deseja-se obter medidas, como por exemplo, o grau de estenose. Porém, as suas funcionalidades são limitadas devido à dificuldade de desenvolverem-se metodologias que abranjam a maior quantidade de cenários clínicos.

Nesta proposta, métodos de caracterização de redes vasculares são investigados para a quantificação de imagens de angiografia 3D, bem como para a própria validação do método de representação. O uso da representação em grafos e cadeias de texto deverá diminuir drasticamente a complexidade de análise computacional. Também permitirá a aplicação de métodos de extração e análise de características baseados em mineração de textos e alinhamentos entre cadeias. O fluxo completo do trabalho é apresentado na Figura 1.2.

Com o desenvolvimento da metodologia proposta pretende-se proporcionar futuras soluções para análise de redes vasculares, o que permitiria prover informações consistentes para uma maior gama de aplicações. 
Figura 1.2: Diagrama de funcionamento da metodologia proposta.
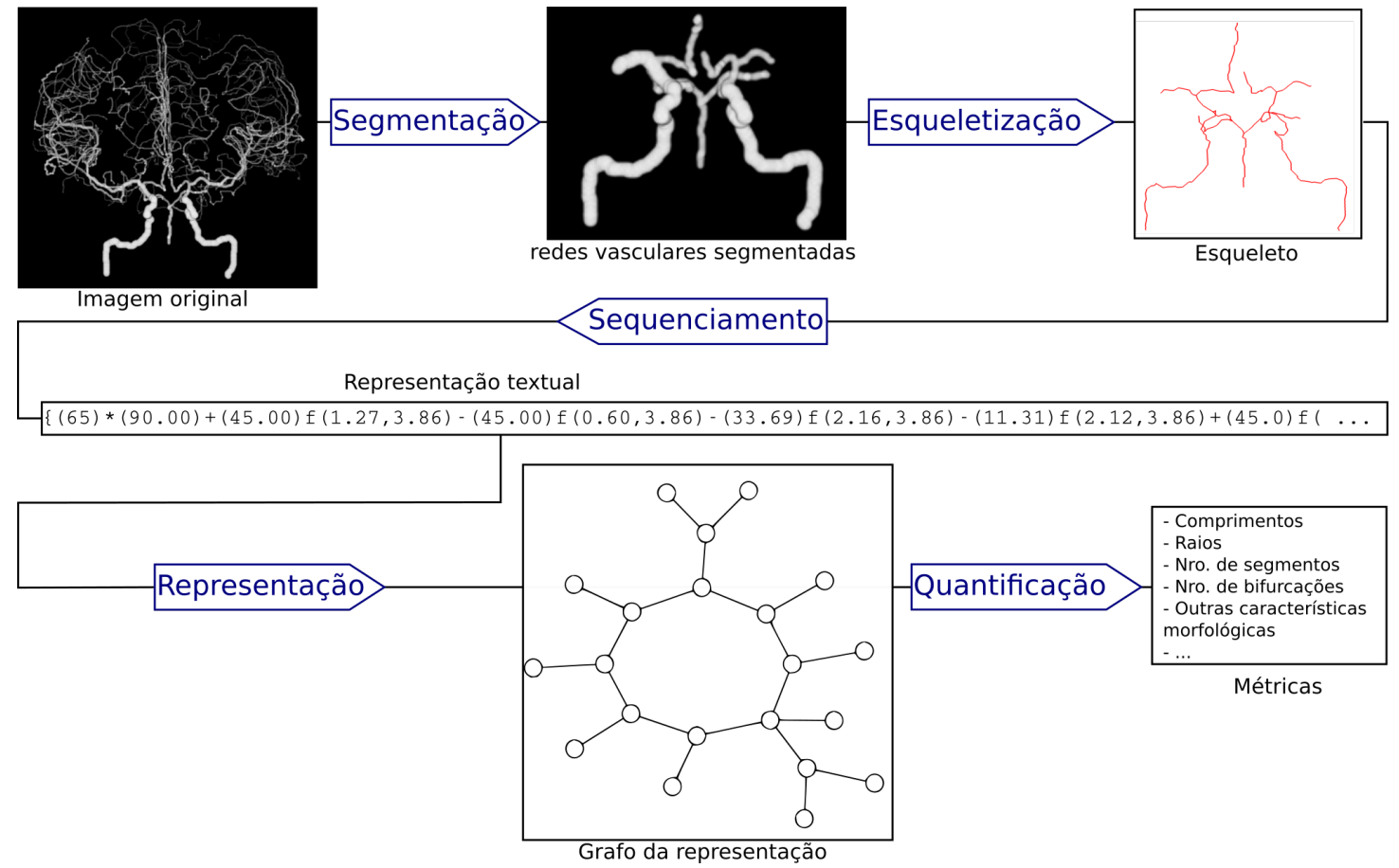

\subsection{Objetivos}

Este trabalho tem por objetivo geral desenvolver uma metodologia de modelagem, caracterização e quantificação de vasos sanguíneos, a partir de imagens tridimensionais de angiografia.

\subsubsection{Objetivos específicos}

Para atingir o objetivo principal foi necessário definir os seguintes objetivos específicos:

- permitir a extração dos vasos e suas trajetórias a partir de imagens tridimensionais de angiografia.

- estabelecer uma linguagem para a representação dos segmentos de redes vasculares em forma textual que permitam estruturas vasculares cíclicas.

- usar as trajetórias para extrair a linguagem representativa de cada segmento e modelar o grafo da rede vascular.

- derivar métricas para a quantificação dos vasos a partir da representação obtida. 
- quantificar as redes vasculares a partir das métricas extraídas, possibilitando a realização de análises comparativas entre redes vasculares.

\subsection{Aplicações}

A metodologia proposta pode ter várias aplicações, por exemplo:

- a detecção e rotulação de vasos (tarefa bem difundida na literatura), na qual procura-se detectar segmentos de rede vascular e atribuir-lhes seu nome anatômico.

- o auxilio no diagnóstico de doenças tais como aneurisma e estenose.

- o estudo das mudanças da estrutura vascular com a idade.

- a análise da evolução dos vasos depois de receber tratamentos.

- a prevenção de traumatismos, tais como a dissecção coronária espontânea, uma vez que o aumento da tortuosidade na aorta pode ser um indicativo de predisposição à doença [Eleid et al., 2014].

\subsection{Organização do trabalho}

No Capítulo 2 a seguir discorre-se sobre o estado da literatura em relação à quantificação de redes vasculares. No Capítulo 3 faz-se uma recopilação dos principais conceitos usados na metodologia, a qual é apresentada no Capítulo 4. Na sequência, no Capítulo 5 são mostrados resultados obtidos em cada uma das etapas da nossa metodologia. Finalmente, o Capítulo 6, é dedicado à discussão de nossos resultados e à sugestão de trabalhos futuros. 


\section{Capítulo 2}

\section{Trabalhos correlatos}

Neste capítulo, é feita uma revisão sistemática da literatura, elaborada com o intuito de apresentar um levantamento do estado da arte bem como identificar possíveis lacunas dos métodos usados no processo de quantificação vascular.

Uma revisão sistemática, tal como descrita em Keele [2007], é um meio de avaliar e interpretar todas as investigações relevantes disponíveis para um dado tópico de investigação ou fenômeno de interesse.

Esta revissão foi realizada considerando as três fases fundamentais: Planejamento, Realização e Reporte da revisão. Essas fases foram sugeridos nos procedimentos para a realização de revisões sistemáticas propostas em [Kitchenham, 2004].

\subsection{Planejamento da revisão}

Nesta revisão, foi usada a seguinte configuração:

Objetivo: Identificar as principais representações computacionais de redes vasculares e os métodos mais usados no processo de sua quantificação.

Fontes: Foram consultadas as seguintes fontes:

Scopus, PubMed, Web of Science e IEEE Xplore. 


\subsubsection{Método de busca}

Foi realizada uma busca na literatura nos artigos publicados entre os anos 2006 e 2017 nos repositórios listados anteriormente. Essa busca foi realizada com as seguintes palavras chave: quantification, blood vessel, morphology e 3-D. Adicionalmente, escolhemos alguns termos de busca relacionados às palavras chave, tais como: characterization, angiography, architecture, three dimensional. Por outro lado, com o intuito de limitar os resultados e focar os esforços em estruturas 3-D, desconsideramos os artigos relacionados a técnicas como microscopia, ultrassom, IVUS ${ }^{1}$ e $\mathrm{OCT}^{2}$, as quais produzem imagens que não possuem a mesma natureza daquelas consideradas neste trabalho.

Finalmente, a cadeia de busca usada nesta revisão da literatura foi:

(angiography OR blood vessel) AND (characterization OR quantification) AND (three dimensional OR 3-D) AND (morphology OR architecture) AND NOT (microscopy OR microscopic OR oct OR ultrasound OR ivus)

Dado que os repositórios de artigos usados não possuem um protocolo nem linguagem padronizada de busca, fez necessário o uso de diferentes cadeias de busca, as quais são mostradas na Tabela 2.1.

A lista de referencias obtida foi analisada e a ela foram aplicadas os seguintes critérios de exclusão:

i) O artigo não é acessível de forma integral em sites de publicações científicas;

ii) $\mathrm{O}$ artigo se presta a uma revisão da literatura;

iii) O estudo não é relacionado à caraterização ou quantificação da morfologia de redes vasculares.

iv) $\mathrm{O}$ artigo foca em técnicas que geram imagens que não compartilham a mesma natureza das geradas pela angio-RM ou angio-CT.

\footnotetext{
${ }^{1}$ IVUS: Ultrassom Intra-Vascular

${ }^{2}$ OCT: Optical Coherence Tomography
} 
Tabela 2.1: Cadeias de busca usadas nos diferentes repositórios

\begin{tabular}{|c|c|}
\hline Repositório & Cadeia de busca \\
\hline IEEE Xplore & $\begin{array}{l}\text { ("angiography"OR "blood vessel") AND ("quantification"OR "characterization") } \\
\text { AND ("3-D"OR "three dimensional") AND ("morphology"OR "architecture") AND } \\
\text { NOT ("microscopy"OR "microscopic"OR "oct"OR "ultrasound"OR "ivus") }\end{array}$ \\
\hline Scopus & 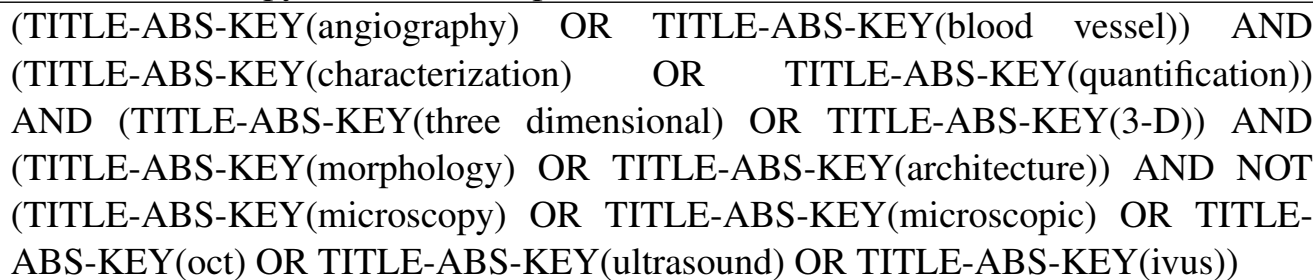 \\
\hline WoS & $\begin{array}{l}\mathrm{TS}=((\text { angiography OR blood vessel) AND (characterization OR quantification) } \\
\text { AND (three dimensional OR 3-D) AND (morphology OR architecture) }) \text { NOT } \\
\text { TS=(microscopy OR microscopic OR oct OR ultrasound OR ivus) }\end{array}$ \\
\hline PubMed & $\begin{array}{l}\text { ("angiography"[All Fields] OR "blood vessel"[All Fields]) AND ("quantification"[All } \\
\text { Fields] OR "characterization"[All Fields]) AND ("3-D"[All Fields] OR "three dimen- } \\
\text { sional"[All Fields]) AND ("morphology"[All Fields] OR "architecture"[All Fields]) } \\
\text { NOT ("microscopy"[All Fields] OR "microscopic"[All Fields] OR "oct"[All Fields] } \\
\text { OR "ultrasound"[All Fields] OR "ivus"[All Fields]) }\end{array}$ \\
\hline
\end{tabular}

\subsection{Realização da revisão}

\subsubsection{Seleção de artigos}

Realizamos a busca nas fontes listadas anteriormente, usando as cadeias mostradas na Tabela 2.1. Os resultados dessas buscas foram filtrados de tal forma que os artigos publicados entre os anos 2006 e 2017 fossem mantidos. Essa busca gerou um total de 193 artigos, dos quais após remover duplicações, obtivemos 113 artigos. Dentre os artigos que conseguimos acessar, 77 foram excluídos de acordo com nossos critérios de exclusão após a revisão dos resumos dos artigos. Na sequência, dentre os 40 artigos selecionados até esta etapa, excluímos trabalhos considerados como revisões da literatura, bem como trabalhos nos quais as características dos vasos tenham sido extraídas apenas por procedimentos mecânicos, e ainda trabalhos que focassem no estudo de características que não pudessem ser obtidas a partir de imagens de angio-RM ou angio-TC. Finalmente, 22 artigos foram selecionados para análise. Todo esse fluxo é mostrado na Figura 2.1. Dito fluxo foi baseado na estrutura sugerida pelo grupo PRISMA [Liberati et al., 2009] e elaborado para melhorar e padronizar a elaboração de revisões sistemáticas. 
Figura 2.1: Fluxo da análise de artigos baseado no PRISMA 2009 [Liberati et al., 2009]
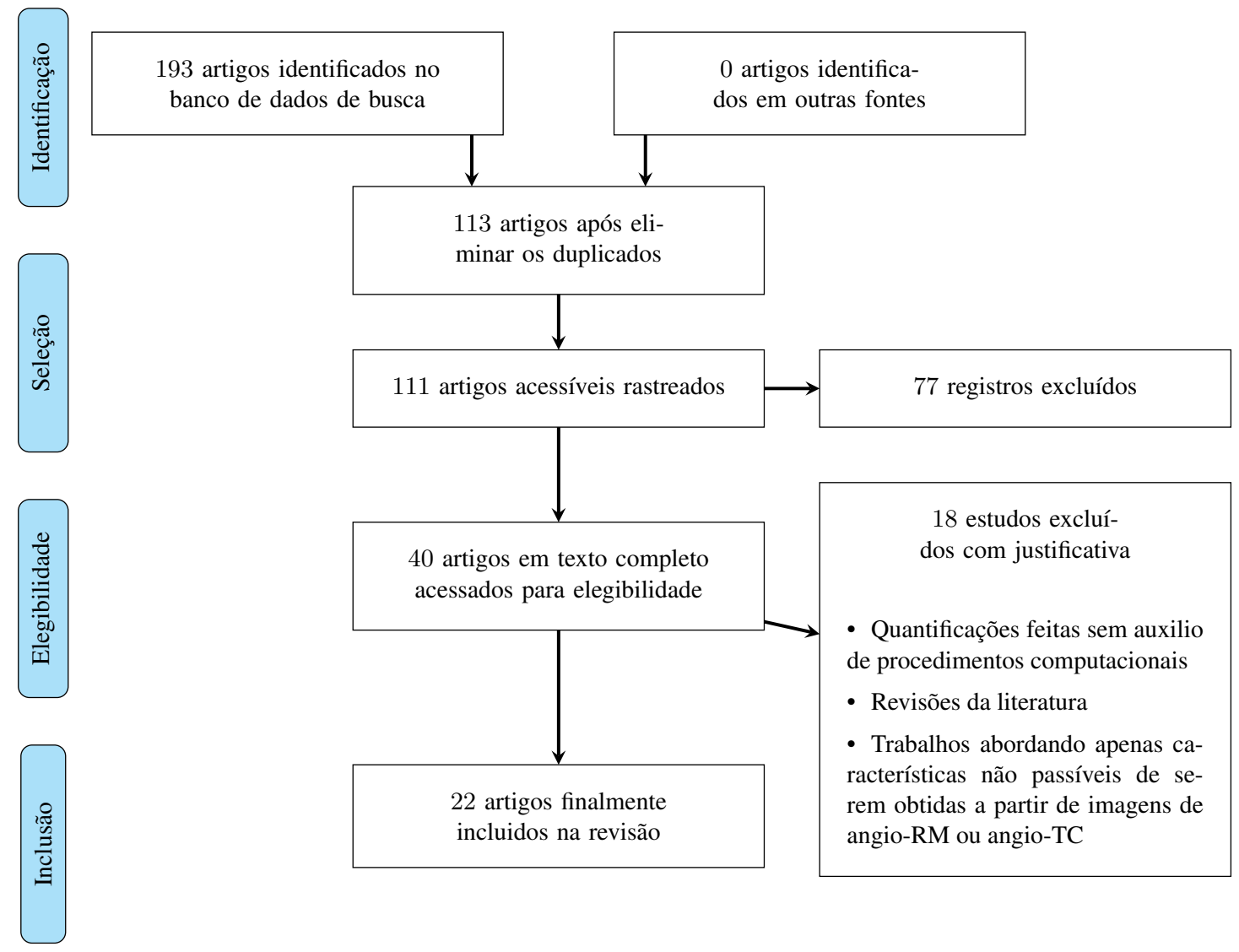


\subsection{Revisão}

Os 22 artigos selecionados foram revisados; suas características mais destacadas foram extraídas e são mostradas nas Tabelas 2.2 e 2.3. Nela se apresentam as informações que consideramos mais relevante em cada um dos artigos selecionados. Em ambas as tabelas, na coluna intitulada Representação do vaso mostra-se a representação intermediária a partir da qual os autores extraem as características dos vasos ou os quantificam.

$\mathrm{Na}$ coluna intitulada Características descreve-se de forma sucinta as características que os trabalhos usam ou extraem a partir dos vasos; nesta coluna alguns nomes de características foram padronizados. Na coluna Aplicação é mencionado o objetivo do trabalho analisado. $\mathrm{Na}$ coluna Segmentação são listados os métodos usados para a segmentação das imagens usadas (registramos o símbolo "-" nos trabalhos que não descreveram os métodos de segmentação, ou no caso que o estudo usasse segmentações prontas). A coluna Admite ciclos sinaliza se os trabalhos apresentaram resultados com segmentos formando ciclos, ou se descrevem o comportamento das suas metodologias em estruturas cíclicas. Finalmente, na coluna Esqueletização são destacados os trabalhos cujos métodos descrevem uma esqueletização como parte do pré-processamento ou na caracterização dos vasos.

Dos artigos selecionados na revisão, apenas seis não usam uma representação intermediária das redes vasculares sendo que a extração das características se da a partir das imagens originais ou de sua segmentação, fato que pode trazer como consequência o processamento repetitivo da imagem inicial, que gera um consumo de tempo computacional desnecessário. Em outros nove artigos a representação dos vasos é feita por meio de superfícies geométricas, a partir das quais são extraídas características. Essa técnica requer o processamento destas superfícies, o que, dependendo da quantidade de vértices e arestas que as compõem, pode ser custoso computacionalmente.

O trabalho de Heinzer et al. [2008] usa a estrutura de grafos genéricos, e não apenas árvores, para representar redes vasculares de modelos murinos. Os autores desse trabalho geram um grafo cujos vértices são os pontos de bifurcação e as arestas as conexões entre eles. Neste trabalho faz-se o uso do esqueleto dos vasos para a extração das características dos mesmos, as quais, em sua maioria, são baseadas em contagem e análise de voxels. Seus resultados são promissores e mostram a viabilidade do uso dos grafos como mecanismo de representação. Porém, caso seja requerida informação do diâmetro dos segmentos (úteis para calcular o volume ou o área

\footnotetext{
${ }^{a}$ AAA: Aneurisma da Aorta Abdominal

${ }^{b}$ DSA: Angiografia de substração digital

${ }^{c}$ Software Mimics Materialise, Sheffield, UK
} 
Tabela 2.2: Artigos resultantes da revisão sistemática (parte 1).

\begin{tabular}{|c|c|c|c|c|c|c|c|}
\hline Artigo & $\begin{array}{l}\text { Modelagem } \\
\text { usada }\end{array}$ & $\begin{array}{l}\text { Características } \\
\text { destacadas }\end{array}$ & $\begin{array}{l}\text { Método } \\
\text { de Aqui- } \\
\text { sição }\end{array}$ & Aplicação & Segmentação & $\begin{array}{l}\text { Admite } \\
\text { ciclos }\end{array}$ & $\begin{array}{l}\text { Esque- } \\
\text { letiza- } \\
\text { ção }\end{array}$ \\
\hline Cao et al. [2017] & - & $\begin{array}{l}\text { Tortuosidade, } \\
\text { número e densidade } \\
\text { de segmentos, } \\
\text { conectividade }\end{array}$ & $\begin{array}{l}\text { micro- } \\
\mathrm{CT}\end{array}$ & $\begin{array}{l}\text { Quantificação } \\
\text { do } \\
\text { comportamento } \\
\text { da vasculatura } \\
\text { em ferimentos }\end{array}$ & Limiarização & & $\checkmark$ \\
\hline $\begin{array}{l}\text { Ellwein et al. } \\
\text { [2016] }\end{array}$ & Superfícies & $\begin{array}{l}\text { Tortuosidade, } \\
\text { curvatura, ângulo } \\
\text { de bifurcação, } \\
\text { comprimento, } \\
\text { excentricidade, razão } \\
\text { de Murray }\end{array}$ & $\mathrm{CT}$ & $\begin{array}{l}\text { Quantificação } \\
\text { da artéria } \\
\text { coronária }\end{array}$ & ITK-snap & & $\checkmark$ \\
\hline $\begin{array}{l}\text { Rahaghi et al. } \\
\text { [2016] }\end{array}$ & Árvores & Tortuosidade, volume & CTA & $\begin{array}{l}\text { Caracterização } \\
\text { da morfologia } \\
\text { dos vasos na } \\
\text { doença CTEPH }\end{array}$ & $\begin{array}{l}\text { Procedimentos } \\
\text { mecânicos }\end{array}$ & & \\
\hline $\begin{array}{l}\text { Wolterink et al. } \\
\text { [2016] }\end{array}$ & - & $\begin{array}{l}\text { Volume, medida de } \\
\text { Agatson, índice de } \\
\text { massa }\end{array}$ & $\mathrm{CT}$ & $\begin{array}{l}\text { Classificação } \\
\text { automática }\end{array}$ & - & & \\
\hline $\begin{array}{l}\text { Valverde et al. } \\
\text { [2015] }\end{array}$ & $\begin{array}{l}\text { Sequência } \\
\text { de } \\
\text { instruções }\end{array}$ & $\begin{array}{l}\text { Ordem de Strahler, } \\
\text { taxonomia de } \\
\text { Tokunaga, matriz de } \\
\text { Tokunaga, razão de } \\
\text { bifurcação }\end{array}$ & phantom & $\begin{array}{l}\text { Quantificação } \\
\text { de redes } \\
\text { vasculares 3D }\end{array}$ & - & & $\checkmark$ \\
\hline $\begin{array}{l}\text { Dehmeshki et al. } \\
\text { [2014] }\end{array}$ & - & Comprimento, raio & $\begin{array}{l}\text { CTA, } \\
\text { phan- } \\
\text { tom }\end{array}$ & $\begin{array}{l}\text { Detecção e } \\
\text { caracterização } \\
\text { da doença } \\
\text { arterial } \\
\text { periférica }\end{array}$ & $\begin{array}{l}\text { Limiarização, } \\
\text { crescimento } \\
\text { de regiões, } \\
\text { operações } \\
\text { morfológicas }\end{array}$ & & $\checkmark$ \\
\hline $\begin{array}{l}\text { Wright et al. } \\
\text { [2013] }\end{array}$ & Cilindros & $\begin{array}{l}\text { Comprimento, } \\
\text { caminho máximo, } \\
\text { distancia, amplitude de } \\
\text { Bifurcação (local e } \\
\text { remoto), inclinação, } \\
\text { curvatura, dimensão } \\
\text { fractal, níveis de } \\
\text { bifurcação }\end{array}$ & MRA & $\begin{array}{l}\text { Criar uma } \\
\text { reconstrução } \\
\text { digital das } \\
\text { artérias } \\
\text { cerebrais e } \\
\text { quantificá-las }\end{array}$ & - & & \\
\hline $\begin{array}{l}\text { Duan et al. } \\
\text { [2013] }\end{array}$ & - & $\begin{array}{l}\text { Diâmetro, direção, } \\
\text { torção, índice de } \\
\text { estenose, área, textura }\end{array}$ & $\begin{array}{l}\text { micro- } \\
\mathrm{CT}\end{array}$ & $\begin{array}{l}\text { Quantificar } \\
\text { vasos para } \\
\text { identificar } \\
\text { fibrose hepática }\end{array}$ & $\begin{array}{l}\text { Software } \\
\text { Amira }\end{array}$ & & $\checkmark$ \\
\hline $\begin{array}{l}\text { Neugebauer } \text { et al. } \\
\text { [2013] }\end{array}$ & Superfícies & Área, diâmetro & $\begin{array}{l}\text { CTA e } \\
\text { MRA }\end{array}$ & $\begin{array}{l}\text { Caracterização } \\
\text { de aneurismas }\end{array}$ & $\begin{array}{l}\text { Limiarização } \\
\text { e } \\
\text { interatividade }\end{array}$ & & $\checkmark$ \\
\hline $\begin{array}{l}\text { Piccinelli et al. } \\
\text { [2012] }\end{array}$ & Superfícies & $\begin{array}{l}\text { Raio, área da seção } \\
\text { transversal, volume }\end{array}$ & $\begin{array}{l}\text { Malha } \\
\text { dada } \\
\text { como } \\
\text { entrada }\end{array}$ & $\begin{array}{l}\text { Detecção } \\
\text { dos pontos } \\
\text { terminais de } \\
\text { aneurismas }\end{array}$ & - & & \\
\hline $\begin{array}{l}\text { Ghatwary et al. } \\
\text { [2012] }\end{array}$ & - & $\begin{array}{l}\text { Diâmetros, } \\
\text { comprimento, volume, } \\
\text { área, tortuosidade, } \\
\text { ângulo de bifurcação, } \\
\text { volume, índice de } \\
\text { calcificação }\end{array}$ & CTA & $\begin{array}{l}\text { Caracterização } \\
\text { de aneurismas } \\
\text { na aorta } \\
\text { abdominal }\end{array}$ & $\begin{array}{l}\text { Semi- } \\
\text { automático }\end{array}$ & & \\
\hline $\begin{array}{l}\text { Biesdorf et al. } \\
\text { [2012] }\end{array}$ & Superfícies & $\begin{array}{l}\text { Diâmetros, posições } \\
\text { dos esqueletos }\end{array}$ & CTA & $\begin{array}{l}\text { Segmentar } \\
\text { redes } \\
\text { vasculares }\end{array}$ & $\begin{array}{l}\text { Registro } \\
\text { elástico }\end{array}$ & $\checkmark$ & $\checkmark$ \\
\hline
\end{tabular}


Tabela 2.3: Artigos resultantes da revisão sistemática (parte 2).

\begin{tabular}{|c|c|c|c|c|c|c|c|}
\hline Artigo & $\begin{array}{l}\text { Representação } \\
\text { do vaso }\end{array}$ & Características & $\begin{array}{l}\text { Método de } \\
\text { Aquisição }\end{array}$ & Aplicação & Segmentação & $\begin{array}{l}\text { Admite } \\
\text { ciclos }\end{array}$ & $\begin{array}{l}\text { Esque- } \\
\text { letiza- } \\
\text { ção }\end{array}$ \\
\hline $\begin{array}{l}\text { Zhou et al. } \\
\text { [2011] }\end{array}$ & $\begin{array}{l}\text { Esqueleto e } \\
\text { segmentação }\end{array}$ & $\begin{array}{l}\text { Densidade, } \\
\text { diâmetros e } \\
\text { tortuosidade }\end{array}$ & Micro-CT & $\begin{array}{l}\text { Quantificar a } \\
\text { morfologia dos } \\
\text { vasos }\end{array}$ & $\begin{array}{l}\text { Operações } \\
\text { morfológicas }\end{array}$ & & $\checkmark$ \\
\hline $\begin{array}{l}\text { Washington et al. } \\
\text { [2011] }\end{array}$ & Superfícies & $\begin{array}{l}\text { Tortuosidade, } \\
\text { diâmetros, área, } \\
\text { volume, altura do } \\
\text { AAA }^{a} \text {, curvatura, } \\
\text { caraterísticas da } \\
\text { placa }\end{array}$ & $\mathrm{CT}$ & $\begin{array}{l}\text { Co-relacionar a } \\
\text { mecânica das } \\
\text { paredes e a } \\
\text { morfologia de } \\
\text { aneurismas } \\
\text { na aorta } \\
\text { abdominal }\end{array}$ & $\begin{array}{l}\text { Software } \\
\text { AAAVASC }\end{array}$ & & $\checkmark$ \\
\hline $\begin{array}{l}\text { Worz et al. } \\
\text { [2010] }\end{array}$ & Superfícies & $\begin{array}{l}\text { Curvatura, } \\
\text { raio, torção, } \\
\text { comprimentos }\end{array}$ & $\begin{array}{l}\text { MRA, } \\
\text { CTA e } \\
\text { phantom }\end{array}$ & $\begin{array}{l}\text { Quantificação e } \\
\text { restauração da } \\
\text { aorta }\end{array}$ & $\begin{array}{l}\text { Modelos } \\
\text { deformáveis }\end{array}$ & & $\checkmark$ \\
\hline $\begin{array}{l}\text { Biesdorf et al. } \\
\text { [2009] }\end{array}$ & - & $\begin{array}{l}\text { Direção, raio, } \\
\text { número de } \\
\text { bifurcações }\end{array}$ & $\begin{array}{l}\text { CTA, } \\
\text { phantom }\end{array}$ & $\begin{array}{l}\text { Segmentação e } \\
\text { caracterização } \\
\text { do arco da } \\
\text { aorta }\end{array}$ & $\begin{array}{l}\text { Limiarização } \\
\text { e operações } \\
\text { morfológicas }\end{array}$ & & $\checkmark$ \\
\hline $\begin{array}{l}\text { Piccinelli et al. } \\
\text { [2009] }\end{array}$ & Superfícies & $\begin{array}{l}\text { Curvatura, } \\
\text { tortuosidade, } \\
\text { ângulos de } \\
\text { bifurcação, diâmetro }\end{array}$ & CT, MRI & $\begin{array}{l}\text { Análise da } \\
\text { estrutura } \\
\text { vascular }\end{array}$ & $\begin{array}{l}\text { Modelos } \\
\text { deformáveis, } \\
\text { level-sets }\end{array}$ & & $\checkmark$ \\
\hline $\begin{array}{l}\text { Kang et al. } \\
\text { [2009] }\end{array}$ & Esqueleto & $\begin{array}{l}\text { Comprimento, } \\
\text { energia de restrição, } \\
\text { diâmetro }\end{array}$ & $\begin{array}{l}\text { Phantoms } \\
\text { e DSA }{ }^{b}\end{array}$ & $\begin{array}{l}\text { Quantificar } \\
\text { segmentos de } \\
\text { artérias }\end{array}$ & $\begin{array}{l}\text { Crescimento } \\
\text { de regiões }\end{array}$ & & $\checkmark$ \\
\hline $\begin{array}{l}\text { Marxen et al. } \\
\text { [2009] }\end{array}$ & $\begin{array}{l}\text { Esqueleto e } \\
\text { segmentação }\end{array}$ & $\begin{array}{l}\text { Ordem de Strahler e } \\
\text { índice de volume }\end{array}$ & Micro-CT & $\begin{array}{l}\text { Analise e } \\
\text { modelo de } \\
\text { vasos pela } \\
\text { ordem de } \\
\text { volume }\end{array}$ & Limiares & & $\checkmark$ \\
\hline $\begin{array}{l}\text { Heinzer et al. } \\
\text { [2008] }\end{array}$ & Grafo & $\begin{array}{l}\text { Comprimento, raios } \\
\text { e tortuosidade de } \\
\text { cada segmento, } \\
\text { densidade de } \\
\text { bifurcações, grau de } \\
\text { junção, densidade de } \\
\text { junções }\end{array}$ & Micro-CT & $\begin{array}{l}\text { Caracterizar } \\
\text { redes } \\
\text { vasculares no } \\
\text { cérebro de } \\
\text { ratos }\end{array}$ & $\begin{array}{l}\text { Filtros de } \\
\text { detecção de } \\
\text { bordas }\end{array}$ & $\checkmark$ & $\checkmark$ \\
\hline $\begin{array}{l}\text { O'Flynn et al. } \\
\text { [2007] }\end{array}$ & Superfícies & $\begin{array}{l}\text { Ângulos de } \\
\text { bifurcação, } \\
\text { tortuosidade, } \\
\text { curvatura, } \\
\text { comprimento }\end{array}$ & MRA & $\begin{array}{l}\text { Caracterizar } \\
\text { a geometria } \\
\text { 3-D arterial } \\
\text { periférica }\end{array}$ & $\begin{array}{l}\text { Software } \\
\text { Mimics }^{c} \text { e } \\
\text { correções } \\
\text { manuais }\end{array}$ & & $\checkmark$ \\
\hline $\begin{array}{l}\text { Huang et al. } \\
\text { [2006] }\end{array}$ & Superfícies & $\begin{array}{l}\text { Comprimento, } \\
\text { volume e raio }\end{array}$ & $\begin{array}{l}\text { Modelos } \\
\text { geomé- } \\
\text { tricos } \\
\text { prontos }\end{array}$ & $\begin{array}{l}\text { Visualização, } \\
\text { armazena- } \\
\text { mento e análise } \\
\text { de RVs }\end{array}$ & - & $\checkmark$ & \\
\hline
\end{tabular}


transversal em um dado ponto), é necessária a análise na imagem original e auxiliados pelo esqueleto.

Também o trabalho de Wright et al. [2013] merece destaque especial, pois simplifica a modelagem das redes vasculares ao usar cilindros. Esse tipo de modelagem mostra-se útil, pois é possível quantificar redes vasculares através de dezenas de características. Essas características são extraídas com o auxilio de uma ferramenta chamada L-measure [Scorcioni et al., 2008], a qual é amplamente usada na área de neuro-morfologia; a L-measure permite extrair 43 métricas morfológicas a partir de reconstrução de neurônios. No entanto, essa metodologia requer a criação de um modelo, o qual é obtido por meio da detecção de pontos destacados dentro do vaso. Esses pontos são usados para definir os limites inicial e final dos cilindros que compõem seu modelo. Esse processo é levado a cabo por meio de procedimentos manuais que envolvem a interação de pessoas especializadas com a imagem. Destacamos ainda com relação aos procedimentos descritos nesse trabalho, o fato de que não permitem analisar estruturas cíclicas, já que a caracterização é feita usando métodos criados originalmente para a análise de neurônios, que são formadas por dendritos que tem estrutura de árvore [Cuntz et al., 2007; Scott e Luo, 2001].

As características usadas nos diferentes trabalhos variam dependendo da sua aplicação, e muitos deles consideram métricas diferentes para uma mesma característica. Por exemplo, para mensurar a tortuosidade, [Washington et al., 2011; Worz et al., 2010] usa a métrica de distancia (DM); em outros casos a tortuosidade é medida como a área entre o segmento e a linha reta limitada pelo primeiro e último vóxel Zhou et al. [2011]. Em casos como estes consideramos todas as medidas como relativas à mesma característica, independentemente do método de sua obtenção. Dessa forma, características como: comprimento máximo, comprimento mínimo, comprimento médio e soma dos comprimentos, seriam consideradas como "comprimento".

As caraterísticas mais usadas entre os trabalhos analisados são mostradas na Figura 2.2. Nela, podemos ver que as características mais usadas são o raio, a tortuosidade e o comprimento. Nessa tabela não são mostradas características utilizadas em apenas um dos trabalhos analisados. Assim, características que tenham sido usadas em apenas um trabalho em particular foram incluídas no item "outros"; são as que listamos a seguir: ordem de Tokunaga, caminho máximo, distância euclidiana, inclinação, trajetória do esqueleto, número de bifurcações, energia de restrição, ordem de volume, dimensão fractal, níveis de bifurcação, textura e assimetria do lúmen. Quanto às características que não se referem à morfologia dos vasos ou que foram usadas apenas para um tipo de aplicação em particular, foram descartadas: medidas da placa, medidas do saco do aneurisma e volume do trombo, entre outras.

Algumas outras características, derivadas das descritas não foram incluídas, por exemplo, 
Figura 2.2: Características mais usadas

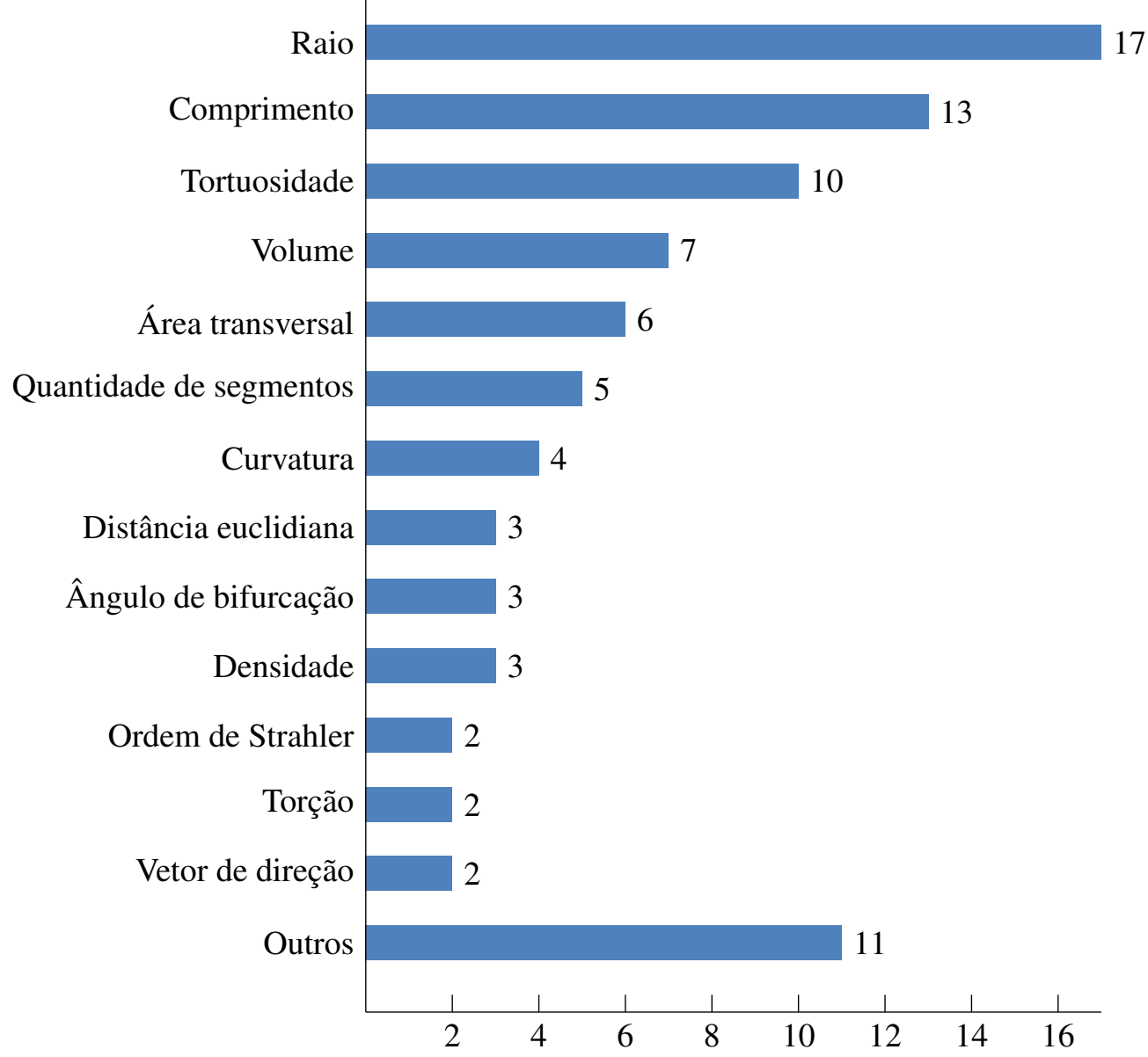

consideramos as características de diâmetro e raio como sendo apenas raio, já que os dois valores são dependentes. Da revisão da literatura que realizamos, omitimos também algumas características que requerem informação adicional ou que são muito específicas. Por exemplo, a Altura do Aneurisma da Artéria Abdominal (AAA), usada por Washington et al. [2011]. Também não foram consideradas as características que não são parte da morfologia dos vasos, por exemplo, a textura usada por Duan et al. [2013], ou características extraídas a partir da segmentação da placa formada dentro dos vasos [Washington et al., 2011]. 



\section{Capítulo 3}

\section{Conceitos preliminares}

\subsection{Imagens médicas}

As imagens médicas são importantes ferramentas de auxílio no processo de diagnóstico de doenças de diferentes naturezas. Na radiologia, destacam-se as imagens de angiografia, que são usualmente obtidas pelos métodos de angio-TC e angio-RM, métodos que substituem as técnicas de aquisição de angiografia por cateterismo [Rubin e Rofsky, 2012, p. 3].

O tamanho das imagens de angio-TC e angio-RM dependem da resolução espacial estabelecida durante a aquisição. As imagens obtidas podem ser de natureza tridimensional, em cujo caso são formadas por elementos denominados de voxels. Eles definem a resolução espacial nos três eixos, e recebem um valor de intensidade que depende do tecido, ou material, que o voxel representa. No caso das imagens de angiografia, elas facilitam a visualização dos vasos sanguíneos, uma vez que os voxels relativos a eles recebem uma intensidade destacada com relação aos outros tecidos.

\subsubsection{Coordenadas do voxel e do objeto}

Embora um voxel corresponde a uma região do espaço físico no mundo real, suas coordenadas dependem do processo de aquisição e usualmente não são iguais às coordenadas do voxel na imagem, como mostrado no exemplo da Figura 3.1. Para realizar a transformação entre esses espaços de coordenadas é necessária uma matriz afim $(A)$, a qual é usualmente provista pelo escâner. Quando essa informação não é incluída na imagem, usa-se uma matriz identidade. Assim, para transformar coordenadas de imagem em coordenadas de objeto é necessário multiplicar as 
coordenadas pela matriz afim $A$. No sentido oposto, a transformação de coordenadas de objeto para coordenadas de imagem é feita usando a inversa da matriz afim $\left(A^{-1}\right)$.

Figura 3.1: coordenadas do objeto $(x, y, z)$ e as coordenadas da imagem $\left(x^{\prime}, y^{\prime}, z^{\prime}\right)$

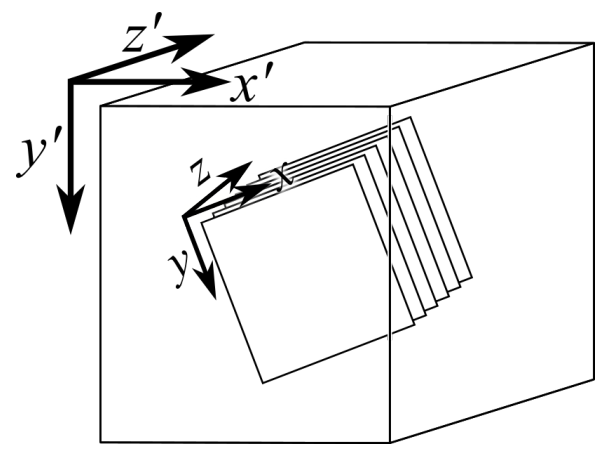

\subsection{Segmentação}

A segmentação, na área de processamento de imagens, é o processo de separação do objeto de interesse do resto da imagem, um processo que é considerado uma ferramenta fundamental na análise de imagens médicas [Bankman, 2008]. No entanto não existe uma técnica definitiva que possa ser usada em todos os contextos e aplicações, e a escolha de um algoritmo de segmentação depende das características da imagem e do objetivo da segmentação, razão pela qual muitos dos métodos de segmentação e de rastreamento de vasos são específicos para determinadas regiões do corpo. Todos esses fatores fazem que o problema de segmentação seja ainda um desafio, sendo um dos tópicos principais das pesquisas nos últimos anos [Lesage et al., 2009; Lu et al., 2017; Pratt et al., 2017]. Entre as abordagens mais conhecidas encontram-se as baseadas em cortes em grafos [Zhu e Chung, 2013], e as baseadas em level sets [Hong e Wang, 2013]. Uma forma de melhorar os resultados é dar destaque aos vasos nas imagens, e um dos métodos mais usados para isto é o método do vesselness.

\subsubsection{Vesselness}

O método de vesselness, inicialmente introduzido em [Frangi et al., 1998], é um filtro que destaca os vasos em imagens de angiografia. $\mathrm{O}$ filtro procura estruturas tubulares na imagem, e atribui maiores valores às regiões cujas intensidades são mais similares a uma estrutura tubular. Isso é conseguido analisando os autovalores da matriz Hessiana das intensidades da imagem. Esses autovalores $\left(\lambda_{1}, \lambda_{2}, \lambda_{3}\right.$, com $\lambda_{1}>\lambda_{2}>\lambda_{3}$, e $\left.\left\|\lambda_{1}\right\|<\left\|\lambda_{2}\right\|<\left\|\lambda_{3}\right\|\right)$ determinam a mag- 
Figura 3.2: Principais direções e os autovalores usados pelo método de vesselness.

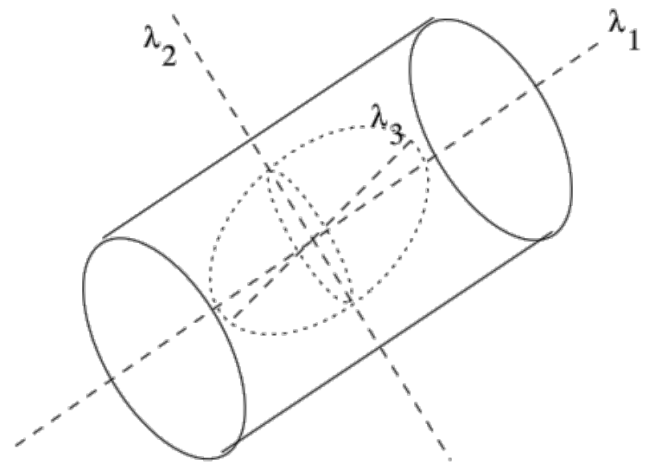

Fonte: Frangi et al. [1998]

nitude do objeto num determinado ponto da imagem. Nesse sentido, voxels correspondentes a vasos sanguíneos são caracterizados por ter um autovalor maior que os outros. Uma representação dos autovalores, apresentada originalmente em [Frangi et al., 1998] é mostrada na Figura 3.2

A função que define o vesselness definida por [Frangi et al., 1998] é:

$$
V(s)= \begin{cases}0 & \text { se } \lambda_{2}>0 \text { ou } \lambda_{3}>0 \\ \left(1-e^{-\frac{R_{A}^{2}}{2 \alpha^{2}}}\right) e^{-\frac{R_{B}^{2}}{2 \beta^{2}}\left(1-e^{-\frac{S^{2}}{2 c^{2}}}\right)} & \text { caso contrário. }\end{cases}
$$

onde,

$$
\begin{gathered}
R_{A}=\frac{\lambda_{2}}{\lambda_{3}} \\
R_{B}=\frac{\lambda_{1}}{\lambda_{2} \lambda_{3}} \\
S=\lambda_{1}^{2}+\lambda_{2}^{2}+\lambda_{3}^{2}
\end{gathered}
$$

Existem algumas implementações do vesselness, uma delas é o filtro de multi-escala [Sato et al., 1997, 1998], que funciona da seguinte forma: são calculados os autovalores $\lambda_{1}, \lambda_{2}$ e $\lambda_{3}$ (com $\lambda_{1}>\lambda_{2}>\lambda_{3}$ ) para cada voxel, e a partir deles é calculada uma medida de similaridade com uma estrutura de linha, dada por:

$$
L=f\left(\lambda_{1}, \lambda_{c}\right) \times \lambda_{c},
$$

Onde $\lambda_{c}=\min \left(-\lambda_{2},-\lambda_{3}\right)$, e $f\left(\lambda_{1}, \lambda_{c}\right)$ é dado por: 


$$
f\left(\lambda_{1}, \lambda_{c}\right)= \begin{cases}\exp \left(-\frac{\lambda_{1}^{2}}{2\left(\alpha_{1} \lambda_{c}\right)^{2}}\right) & \lambda_{1}>0, \lambda_{c} \neq 0 \\ \exp \left(-\frac{\lambda_{1}^{2}}{2\left(\alpha_{2} \lambda_{c}\right)^{2}}\right) & \lambda_{1}>0, \lambda_{c} \neq 0 \\ 0 & \lambda_{c}=0\end{cases}
$$

no qual, $\alpha_{1}$ e $\alpha_{2}$ (com $\alpha_{1}<\alpha_{2}$ ) são parâmetros correspondentes aos valores 0,5 e 2,0, respetivamente, conforme sugestão de Sato et al. [1997].

\subsection{Esqueletização}

A esqueletização é o processo de compactação e redução da dimensionalidade de um objeto numa imagem. No caso das redes vasculares 3D, o esqueleto é formado por curvas 3D que mantêm a topologia original dos vasos. Existem três abordagens principais para a esqueletização [Saha et al., 2016]: as baseadas no algoritmo de Voronoi; as por evolução contínua das bordas; as por erosões morfológicas sucessivas.

Neste processo, se as bordas não forem adequadamente suavizadas, podem produzir-se segmentos que não correspondem a segmentos reais na rede vascular. Esse tipo de erro é muitas vezes tratado com um pós-processamento de poda do esqueleto.

\subsection{Redes vasculares sintéticas}

São imagens geradas artificialmente, de modo que suas caraterísticas imitem às encontradas em imagens de angiografia reais. Sua criação tem sido abordada por diferentes métodos, entre os quais se destacam os baseados em L-systems, e os baseados em crescimento iterativo [Hamarneh e Jassi, 2010].

\subsubsection{Sistemas de Lindenmayer}

Os métodos baseados em L-systems são definidos de acordo com uma gramática que controla a criação de ramificações e torção de segmentos [Valverde, 2012]; já os métodos baseados em crescimento iterativo seguem modelos fisiológicos [Szczerba e Székely, 2002]. Os modelos baseados em L-systems oferecem maior flexibilidade, e permitem a geração de estruturas diferentes apenas mudando sua gramática. Adicionalmente, [Valverde, 2012] apresenta uma extensão aos L-systems, permitindo a inclusão de regras estocásticas para a geração de vasos. 


\subsubsection{Geração de imagens sintéticas com L-systems estocásticos}

O trabalho de Valverde et al. [2013] faz uso de sistemas de Lindenmayer, descrevendo a construção de gramáticas de Lindenmayer estocásticas e paramétricas. Essas gramáticas são compostas por regras que, por sua vez, são formados por elementos terminais e não terminais.

Um $L$-system é uma terna ordenada $G=(\Sigma ; P ; \omega)$, na qual, $\Sigma=s_{1}, s_{2}, \ldots, s_{n}$ é o alfabeto do sistema, $P \subset \Sigma \times \Sigma^{*}$ é o conjunto finito de produções, e $\omega \in \Sigma^{+}$é um elemento não vazio chamado axioma. Uma produção $(a, \chi) \in P$ é escrita como $a \rightarrow \chi$, onde $a$ é chamado predecessor e $\chi$ é o sucessor [Kókai et al., 1999; Prezemyslaw e Lindenmayer, 1996; Rozenberg, 1980].

Exemplo de um L-System básico:

Seja $G=(\{a, b\}, P, a)$, com produções $P=\{a \rightarrow a b, b \rightarrow a\}$

Com a aplicação das definições anteriores, a seguinte sequência de derivação é obtida:

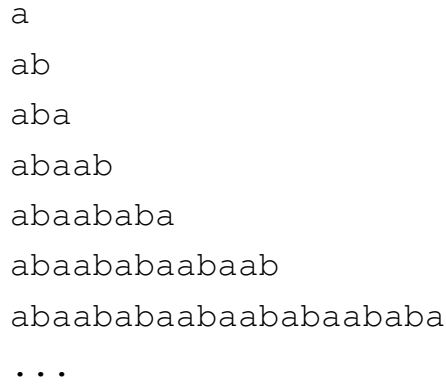

\subsubsection{Variáveis iniciais}

Para a execução da cadeia de instruções, Valverde [2012] define inicialmente alguns parâmetros globais, dentre os quais se destacam:

Posição inicial: a posição inicial é definida como um ponto no espaço cartesiano $P=(x, y, z)$.

Direção: é um vetor unitário $\vec{V}_{\text {direção }}=(\hat{i}, \hat{j}, \hat{k})$ que indica a direção ao próximo segmento.

Direção perpendicular: indica uma direção perpendicular à direção inicial e é representado por um vetor unitário $\vec{V}_{\text {perpendicular }}=(\hat{i}, \hat{j}, \hat{k})$. A definição dos vetores $\vec{V}_{\text {direção }}$ e $\vec{V}_{\text {perpendicular }}$ permite fazer rotações nas 3 dimensões. 


\subsubsection{Caracteres permitidos na representação}

As gramáticas estocásticas de Lindenmayer tal como utilizadas por [Valverde, 2012] usam símbolos que são atrelados a funções. Cada função é representada por uma letra seguida ou não de parâmetros. Por exemplo se a função for $f(4,5)$, significa que a função $f$ será executada com os parâmetros 4 e 5 .

As instruções permitidas na gramática de Valverde [2012] bem como seus respetivos parâmetros, são listados a seguir:

$f$ : Representa avançar em linha reta na direção do vetor $\vec{V}_{\text {direção }}$ atual. Se $f$ não tem parâmetros, esses serão definidos por valores-padrão. A chamada completa ao símbolo $f$ é: $f$ (comprimento, diâmetro), comprimento e diâmetro são números reais que representam o comprimento e o diâmetro do segmento a ser criado pelo símbolo $f$.

+ : Representa uma rotação do vetor atual de direção num dado ângulo com respeito à direção perpendicular em sentido anti-horário.

- : A funcionalidade é igual à do símbolo “+”, mas em sentido horário.

I: Representa uma rotação do vetor $\vec{V}_{\text {perpendicular }}$ num ângulo $\beta$, com respeito ao vetor $\vec{V}_{\text {direção }}$ em sentido horário. Se $\beta$ não for dado como parâmetro, é utilizado um ângulo de 70 graus.

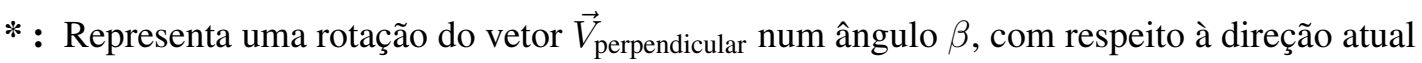
em sentido anti-horário. Se $\beta$ não for dado como parâmetro, é utilizado um ângulo de 70 graus.

[ : Salva o estado atual numa pilha. O estado atual é composto pelos valores de diâmetro, posição e os vetores de direção.

] : Recupera os valores do topo da pilha e define os valores das variáveis a partir dos valores armazenados.

\subsubsection{Geração de vasos sintéticos com L-systems}

A execução da cadeia de instruções é realizada em forma sequencial. Nesse processo cria-se uma sequência de pontos de controle que representa a rede vascular. Esses pontos são unidos posteriormente usando $B$-splines, e finalmente passam por uma etapa de discretização que produze uma imagem sintética tridimensional [Valverde, 2012]. 


\subsubsection{Limitação dos L-systems estocásticos na geração de vasos sintéticos}

Uma das maiores limitações da metodologia de Valverde [2012], tal como descrito por [Ajam et al., 2017], é que apesar de a abordagem produzir resultados visualmente convincentes, a obtenção dos parâmetros e regras para a produção de imagens com a estrutura desejada pode ser uma trabalhosa e entediante tarefa.

Outra limitação deste método, e que se deve ao fato de ele haver sido desenvolvimento especificamente para artérias com estrutura de árvore, é a de que nele a geração de vasos pode produzir erros durante a criação de ciclos.

Neste trabalho, no esforço de gerar uma representação dos vasos que permita quantificá-los, desenvolvemos métodos para a geração de regras de forma automática.

\subsection{Teoria de Grafos}

No processo de análise e quantificação de vasos é comum usar uma estrutura de dados para representar as redes vasculares. Uma das mais usadas é a estrutura de árvore, a qual é um tipo de grafo. Os grafos são coleções de vértices e arestas, e têm uma forte base matemática que os suporta. Neste trabalho, usamos grafos tanto no processo de obtenção de representações, como na etapa de quantificação, motivo pelo qual mencionamos algumas definições importantes a seguir.

\subsubsection{Definições básicas}

Grafo: Um grafo $G$ é uma tupla $(V, E)$, na qual $V$ é o conjunto de vértices e $E$ o conjunto de arestas.

Grafo não-dirigido: Dizemos que o grafo é não dirigido se as arestas não têm uma direção associada.

Passeio: Sequência de vértices, na qual, se $v$ e $w$ são vértices consecutivos, então $v w$ é um arco do grafo.

Caminho: Um caminho, em um grafo não dirigido, é um passeio sem arcos repetidos, e é chamado de caminho simples caso não apresente vértices repetidos. O comprimento do caminho é o número de arcos que o caminho possui. 
Origem e término de um caminho: O primeiro vértice de um caminho é chamado de origem, e o término é o seu último vértice.

Caminho fechado: Um caminho no qual a origem e término correspondem aos mesmos vértices.

Ciclo: Um ciclo, em um grafo não dirigido, é um caminho fechado de comprimento maior que 1.

Grafo conexo: Um grafo é conexo se para cada par de vértices $(x, y \in V)$ existe um caminho com começo em $x$ e término em $y$.

Grau de um vértice: $O$ grau de um vértice $v$, num grafo não dirigido, é a quantidade de arestas que tem pelo menos uma ponta em $v$.

Árvore: Uma árvore é um grafo conexo sem ciclos.

Subgrafo: Um grafo $G^{\prime}=\left(V^{\prime}, E^{\prime}\right)$ é um subgrafo de um grafo $G=(V, E)$, denotado como $G^{\prime} \subseteq G$, se $V^{\prime} \subseteq V$ e $E^{\prime} \subseteq E$.

Subgrafo Induzido: Um subgrafo $G^{\prime}=\left(V^{\prime}, E^{\prime}\right)$ de $G=(V, E)$ é dito induzido se $E^{\prime}=$ $E \cap\left(V^{\prime} \times V^{\prime}\right)$.

Árvore de espalhamento mínima Dado um grafo $G=(V, E)$, dizemos que um subgrafo $T$ de $G$ é uma árvore de espalhamento mínima se as arestas de $T$ são um subconjunto acíclico de $E$, que adicionalmente conecta todos os vértices de $G$. Se as arestas tem pesos, a soma das arestas debe ser minimizada.

Isomorfismo de grafos Dois grafos são isomorfos se existe um mapeamento bijectivo dos vértices de um grafo nos vértices do outro, de tal forma que a conexidade entre eles é mantida.

Isomorfismo de subgrafo Existe um isomorfismo de subgrafo entre dois grafos $G$ e $G^{\prime}$, se $G$ é isomorfo a um subgrafo de $G^{\prime}$.

\subsection{Quantificação}

\subsubsection{Características na literatura}

$\mathrm{Na}$ literatura, existe uma ampla diversidade de características que podem ser extraídas a partir de imagens de angiografia. Dentre as mais usadas nos processos de quantificação de vasos encontram-se: 
- Raio, comprimento dos segmentos e características derivadas como:

- Diâmetro, volume, área de superfície e área transversal.

- Quantidade de segmentos.

- Número de bifurcações.

- Quantidade de pontos terminais.

- Níveis de bifurcação.

- Ângulos de bifurcação.

- Tortuosidade e curvatura, mensurados por:

- Métrica de contagem de pontos de inflexão (Inflection Counting Metric, ICM).

- Métrica de distância (Distance Metric, DM).

- Métrica de soma de ângulos (Sum Of Angles Metric, SOAM)

- Ordem de Strahler.

- Taxonomia de Tokunaga.

\subsubsection{Raios e comprimentos}

O raio de um segmento vascular é a distância do centro do vaso até a borda. Para sua obtenção é comum o cálculo a partir de um corte transversal ao segmento. Quanto ao comprimento, consiste na distância entre o ponto inicial e final de um segmento considerando-se sua trajetória.

Conhecer o comprimento do segmento vascular e os raios ao longo dele é fundamental para a caracterização de vasos, uma vez que estas são medidas básicas para a obtenção de outras medidas derivadas, tais como: o diâmetro, o volume, e a área da superfície.

\subsubsection{Tortuosidade}

Os segmentos da vasculatura no corpo humano são geralmente tortuosos em diferente grau. Por exemplo, a aorta é em grande parte um segmento pouco tortuoso, já as artérias no cérebro apresentam maior tortuosidade. Há ainda os casos em que se encontram segmentos com regiões em espiral. Essa medida pode variar em função de diferenças entre segmentos ou em função 
de diferenças entre indivíduos. É possível, inclusive, observar que algumas doenças são caracterizadas por essa variação de tortuosidade, por exemplo, hemorragias intracerebrais podem ser causadas por pequenos segmentos com alta tortuosidade [Bullitt et al., 2003], e também no caso do lúpus eritematoso sistêmico as artérias apresentam maior tortuosidade [Moritani et al., 2001].

A tortuosidade pode ser quantificada por diversas medidas na literatura. Algumas das mais usadas são apresentadas a seguir:

Métrica de distância (Distance Metric, DM) A DM é uma medida simples que reflete a tortuosidade de um segmento vascular. Em [Bullitt et al., 2003] calcula-se a DM com a Equação (3.4):

$$
D M=\frac{\text { Comprimento total do segmento }}{\text { Distância linear entre o ponto inicial e o ponto final do segmento }}
$$

Métrica de contagem de pontos de inflexão (Inflection Counting Metric, ICM) Geralmente a DM é uma boa indicadora da tortuosidade. Porém, dois segmentos extremamente diferentes podem ter um mesmo valor de $\mathrm{DM}$, por exemplo, um segmento em forma de $\mathrm{S}$ pode ter o mesmo valor que um segmento com uma curva em forma de C. Para evitar esse problema, Bullitt et al. [2003] sugerem o uso da ICM como valor de ponderação da DM.

A ICM é definida como a quantidade de pontos de inflexão em um segmento mais 1. A adição do 1 é feita para evitar que a ICM tenha valor zero. Para a determinação dos pontos de inflexão, no caso de segmentos 3D, é usado o triedro de Serret-Frennet [Hu et al., 2011; Mazur et al., 2015]. O triedro é formado por um vetor Tangente à curva $(T)$, por um vetor Normal $(N)$ perpendicular ao eixo de curvatura e à tangente, e por um vetor Binormal $(B)$ que é perpendicular a $B$ e $T$. A Figura 3.3 mostra um exemplo do triedro de Serret-Frennet.

Bullitt et al. [2003] sugerem que quando o triedro passa por um ponto de inflexão, o vetor Normal rotaciona em um ângulo $a \approx 180^{\circ}$.

Métrica de soma de ângulos (Sum Of Angles Metric, SOAM) A SOAM é uma medida, cujo valor é diretamente proporcional à tortuosidade de um segmento. Esta métrica é calculada por meio da soma de todos os ângulos formados por três pontos de controle consecutivos ao longo de um segmento. Trata-se de uma métrica especialmente efetiva para a detecção de altas frequências, como as apresentadas em espirais ou senoides [Bullitt et al., 2005].

Para o cálculo do valor da SOAM, segundo Bullitt et al. [2003], considera-se um segmento 
Figura 3.3: Triedro de Serret-Frennet.

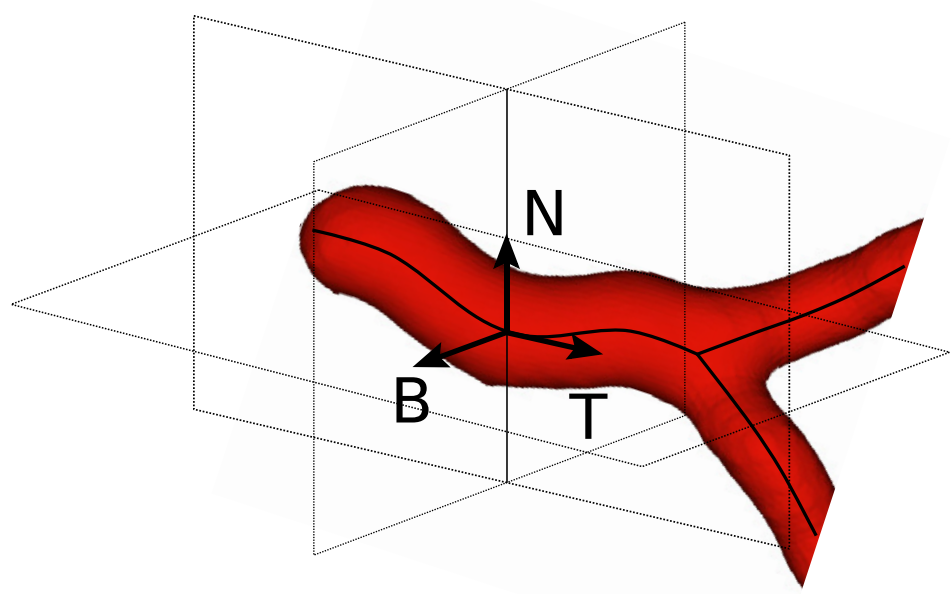

$(S)$ formado por $n$ pontos de controle, $n \geq 3$. Por exemplo, sejam $P_{k-1}, P_{k}$ e $P_{k+1}$, três pontos de controle consecutivos de um segmento, os vetores $T_{1}, T_{2}$ e $T_{3}$ são definidos como:

$$
\begin{aligned}
& T_{1}=P_{k}-P_{k-1}, \\
& T_{2}=P_{k+1}-P_{k}, \\
& T_{3}=P_{k+2}-P_{k+1} .
\end{aligned}
$$

Sejam $\vec{T}_{1}, \vec{T}_{2}$ e $\vec{T}_{3}$ vetores unitários de $T_{1}, T_{2}$ e $T_{3}$. Para um ponto $P_{k}$, o ângulo no ponto $\left(A P_{k}\right)$ e o ângulo de torção $T P_{k}$ são dados por:

$$
\begin{aligned}
& A P_{k}=\cos ^{-1} \vec{T}_{1} \cdot \vec{T}_{2} \\
& T P_{k}=\cos ^{-1}\left(\vec{T}_{1} \times \vec{T}_{2}\right) \cdot\left(\vec{T}_{2} \times \vec{T}_{3}\right) \\
& C P_{k}=\sqrt{A P_{k}^{2}+T P_{k}^{2}}
\end{aligned}
$$

Finalmente, o valor da métrica SOAM para o segmento $(S)$ é:

$$
\operatorname{SOAM}(S)=\frac{C P(S)}{\operatorname{comprimento}(S)}=\frac{\sum_{k=1}^{n-3} C P_{k}}{\sum_{k=1}^{n-1}\left\|P_{k}-P_{k-1}\right\|}
$$




\subsubsection{Características topológicas}

Ordem de Strahler A ordem de Strahler [Strahler, 1957] (também chamada de Horton-Strahler) é uma propriedade que aborda a hierarquia das ramificações [Newman et al., 1997; Zaliapin e Kovchegov, 2012], e foi inicialmente desenvolvida nos estudos da geomorfologia de bacias hidrográficas. Posteriormente foi usada para descrever sistemas de bifurcação em árvores [Zeide e Gresham, 1991] no sistema cardiovascular [Zoghbi et al., 2014], bem como em outras aplicações da medicina [Horsfield, 1980].

De forma geral, o valor de Strahler de um segmento $X \operatorname{com} n$ segmentos filhos $\left(X_{1}, X_{2}, \ldots, X_{n}\right)$, será dado por:

$$
S(X)= \begin{cases}1 & \text { Se } X \text { não tem segmentos filhos }(n=0) . \\ S\left(X_{1}\right)+1 & \text { Se } S\left(X_{1}\right)=S\left(X_{i}\right), \forall i \leq n, n>1 . \\ \text { maior }\left(S\left(X_{i}\right)\right) & \text { Caso contrário. }\end{cases}
$$

Taxonomia de Tokunaga A taxonomia de Tokunaga [Newman et al., 1997; Tokunaga, 1978] foi desenvolvida como uma extensão da ordem de Strahler, permitindo analisar as vizinhanças nas bifurcações. Nesta taxonomia cada segmento é associado a um valor, que por sua vez é composto por dois valores, sendo o primeiro deles o valor de Strahler, e o segundo, um valor calculado da seguinte forma:

Seja um segmento $X$ com pai $X_{p}$; segmentos filhos $X_{1}, X_{2}, \ldots, X_{n}$; e segmentos irmãos (que compartilham o mesmo pai) $X_{h_{1}}, X_{h_{2}}, \ldots, X_{h_{n^{\prime}}}$. O segundo valor de Tokunaga é dado por:

$$
T(X)= \begin{cases}1 & \text { Se } n=0, \text { e } S\left(X_{h_{i}}\right)=1, \forall i \leq n^{\prime} \\ T\left(X_{1}\right) & \text { Se } n=1, \\ T\left(X_{1}\right)+1 & \text { Se } S\left(X_{i}\right)=T\left(X_{j}\right), \forall i, j \leq n \text { e } n>1, \\ \text { maior }\left(T\left(X_{h_{i}}\right)\right) & \text { Caso contrário. }\end{cases}
$$




\section{Capítulo 4}

\section{Metodologia}

Neste capítulo descrevemos nossa metodologia de representação e quantificação de redes vasculares a partir de imagens de angiografia tridimensional, bem como algumas das suas aplicações. Esta metodologia está dividida em três etapas principais: pré-processamento, extração da representação e quantificação.

\subsection{Pré-processamento}

O processo de extração da representação requer imagens resultantes de algum método de segmentação. O sucesso de nosso trabalho de quantificação é em grande medida influenciado pelos resultados de segmentação a partir dos quais é feito o procedimento. Essas segmentações podem ser obtidos por métodos manuais ou por métodos existentes descritos na literatura.

Embora o processo de segmentação não é o objetivo principal do presente trabalho, pelo fato de que requerem-se resultados de segmentação para dar continuidade às etapas de representação e quantificação, foi necessário desenvolver um método de segmentação que permita a obtenção das imagens necessárias para a prática da metodologia.

\subsubsection{Segmentação}

As imagens de angiografia realçam as redes vasculares e atribuem maiores valores à intensidade dos voxels correspondentes a vasos, porém, na maioria das vezes, um limiar não é suficiente para classificar um voxel como sendo parte ou não de um vaso, já que nestas imagens são exibidas não apenas redes vasculares, mas também outras partes do corpo como órgãos, ossos e 
músculos. Por este motivo, usamos um método de realçamento de redes vasculares em imagens chamado de vesselness [Frangi et al., 1998; Sato et al., 1997], descrito na Seção 3.2.1.

Calculamos os valores do vesselness através de um filtro multi-escala [Sato et al., 1997, 1998], o qual requer o cálculo de uma matriz hessiana $\nabla^{2} H\left(x, \sigma_{f}\right)$; essa matriz faz uso de uma função gaussiana isotrópica $f$, com uma dada desviação estândar $\sigma_{f}$.

O filtro destaca os voxels com um perfil similar ao das estruturas tubulares, ou seja com uma anisotropia definida. Porém, dependendo do calibre dos vasos, alguns locais podem apresentar uma alta isotropia, mesmo sendo parte dos vasos. Para evitar o problema, o filtro de Sato et al. [1997] faz uso de equalização de ruido, o que para nossa aplicação mostrou-se insuficiente. Por tal motivo, aplicamos a técnica do vesselness $(L(I, \sigma))$ na imagem original $(I)$ repetidas vezes e com diferentes desvios padrão $\sigma_{s}$, com a eq. (4.1).

$$
\sigma_{s}=\left\{\sigma_{x} \in\left[\sigma_{1}, \sigma_{2}\right]: \sigma_{x}=\sigma_{1}+\operatorname{step} * i, i \in \mathbb{N}\right\}
$$

onde "step" é uma constante. Finalmente, esses resultados são unificados em uma imagem da seguinte forma:

$$
V(I)=\max \left\{L\left(I, \sigma_{i}\right): \sigma_{i} \in \sigma_{s}\right\}
$$

O resultado do processo é uma imagem formada por voxels $\left(v_{x}\right)$ com valores reais $\left(v_{x} \in\right.$ $\mathbb{R})$. Dita imagem destaca os vasos atribuindo intensidades maiores aos voxels que o compõem. Contudo após obtidos esses resultados, resta ainda classificar os voxels por um processo de segmentação.

Para tanto, realizamos um processo de binarização da imagem por meio de um limiar. Na obtenção dos valores dos limiares o uso de algoritmos de limiarização dinâmicos (como os de Otsu e Yen) resultaram em segmentações pouco acurados, o que se deve em grande parte à natureza variável das imagens. Dessa forma, a intervenção do conhecimento do usuário faz-se necessária. Neste contexto, fez se necessário como parâmetro um valor de percentil, o qual determina a porcentagem de voxels que serão considerados como possíveis voxels correspondentes a redes vasculares.

Com o intuito de eliminar o ruído que poderia ter sido criado no resultado obtido, extraímos o máximo elemento conexo da segmentação obtida. Finalmente, prevendo o fato de que dependendo dos valores de desvio padrão usados, os centros dos vasos com maior calibre poderiam ter sido considerados como sendo voxels com intensidades isotrópicas (o que produz pequenos valores de vesselness), aplicamos um filtro de fechamento morfológico e uma transformada ge- 
odésica para o fechamento de buracos [Soille, 2013, p. 208] sobre o resultado. Caso o filtro não tivesse sido aplicado, os baixos valores de vesselness teriam induzido à eliminação de voxels do centro do vaso.

\subsection{Extração da Representação}

Nossa representação baseia-se na cadeia de instruções descrita na metodologia de geração de imagens sintéticas de Valverde [2012], a qual tem como resultado intermediário uma representação em cadeia de texto em que cada caractere tem uma instrução associada. Essa metodologia limita-se à geração de imagens a partir de uma gramática de Lindenmayer, no entanto a obtenção da representação a partir de imagens (processo inverso) é completamente factível.

Figura 4.1: Fluxo representativo da extração de sequências a partir de imagens de angiografia 3D.

Imagem segmentada
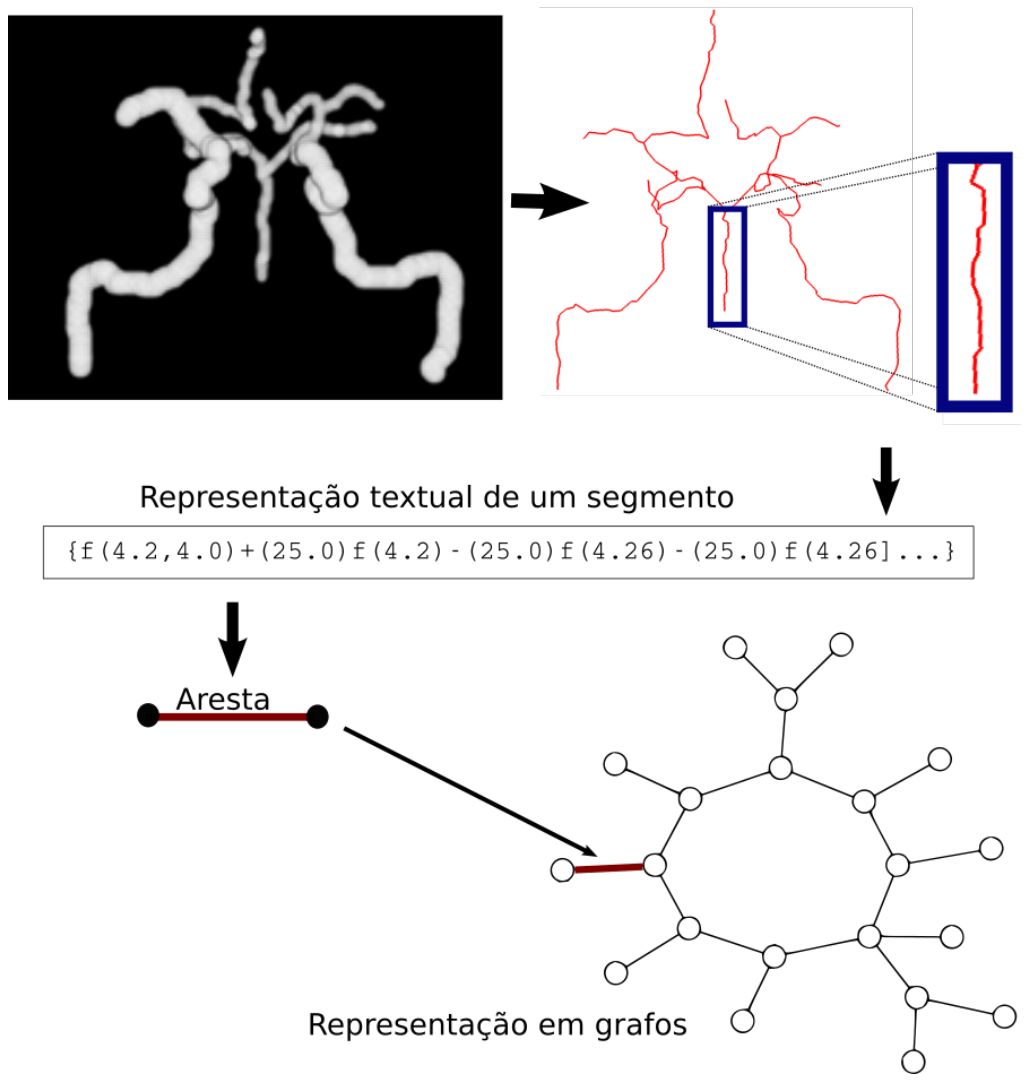

Nesta seção, detalhamos o procedimento de extração da representação vascular, para o qual 
assumimos uma segmentação ótima da rede vascular. Para tanto, extraímos primeiro as linhas centrais do vaso; depois detectamos neles os pontos de bifurcação, os pontos extremos, e pontos de controle nas curvas; na sequência, obtemos o diâmetro do vaso em cada ponto da linha central do vaso; finalmente, armazenamos as instruções de cada segmento em um grafo, o qual, será usado no processo de quantificação. Um fluxo de todo esse processo é mostrado na Figura 4.1.

\subsubsection{Esqueletização}

O esqueleto da rede vascular é obtido a partir de resultados adequados de segmentação, os quais devem ser formados por voxels com valores 0 (quando o voxel é parte do fundo) e 255 (quando o voxel é parte do vaso). Para a extração do esqueleto $L$ a partir de uma imagem segmentada $I$, usamos o método de esqueletização por afinamento (desenvolvido em [Lee et al., 1994]), que garante a não existência de voxels elimináveis, e mantém as condições topológicas e geométricas do objeto analisado. Esse processo gera resultados como o apresentado na Figura 4.2.

Figura 4.2: Exemplo de esqueletização de uma imagem 3D.

(a) Projeção de uma imagem sintética

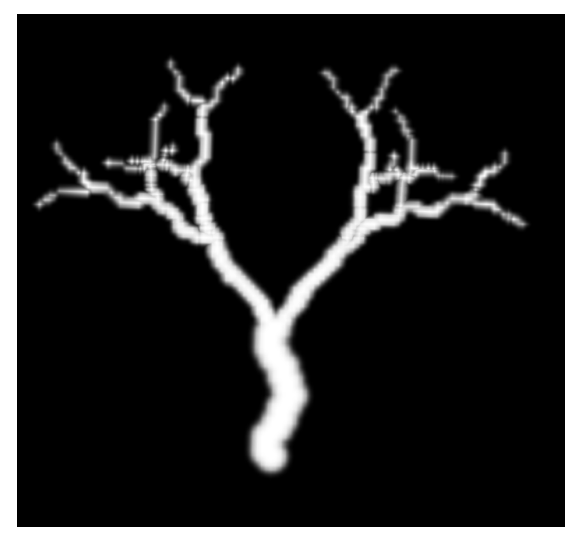

(b) O esqueleto obtido.

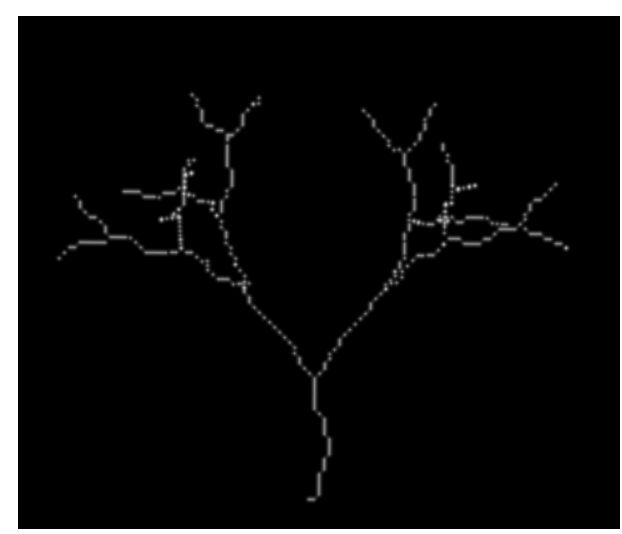

\subsubsection{Cálculo dos raios}

Para obter nossa representação, além do esqueleto necessitamos dos valores dos raios de cada um dos segmentos da rede vascular analisada. Para a obtenção desses valores fazemos uso da transformada de distância [Danielsson, 1980; Soille, 2013]. A transformada gera uma outra imagem tridimensional, cujos voxels possuem valores que representam a distância mínima desse voxel até a borda. Os valores são positivos se o voxel é considerado parte da segmentação 
e negativo se o voxel for parte do fundo da imagem. Vale ressaltar que as distâncias obtidas pela transformada são medidas por meio da quantidade de voxels. Estes valores têm que ser convertidos em milímetros. Para tanto, faz-se uma conversão que leva em conta o espaçamento da imagem segmentada. Finalmente, consideramos o valor atribuído a cada voxel pertencente ao esqueleto como o raio do segmento nesse dado voxel.

O processo de segmentação no entanto pode resultar em uma perda de informação, dado o fato de que as imagens de angiografia são discretizadas e possuem uma resolução limitada. Devido a esses fatores pode-se produzir uma alteração dos raios, em cujo caso a alteração é usualmente de apenas 1 voxel. Esta pequena alteração pode ser significante dependendo da resolução da imagem, de modo que, se obtém melhores resultados se os raios também forem dados como entrada.

\subsubsection{Processamento do esqueleto}

Apesar da esqueletização utilizada no presente trabalho estar baseada em procedimentos amplamente divulgados na literatura, devido a determinados fatores, em particular à existência de ângulos abruptos nas bordas ou à alta proximidade de segmentos, alguns segmentos falsos podem ser criados durante o processo. Como esses falsos segmentos em sua maioria têm comprimentos pequenos, seu número pode ser drasticamente reduzido através da remoção de todos os segmentos com comprimentos menores a um dado valor. Nesse processo de remoção usamos um valor igual ao raio máximo dos segmentos na imagem, o que a principio garantiria a manutenção dos principais segmentos. Por exemplo, se quisermos analisar uma rede vascular que contém segmentos com raio igual a $3 \mathrm{~mm}$, teríamos que desconsiderar os segmentos com comprimentos menores que $3 \mathrm{~mm}$. Para a realização deste e demais procedimentos (como a detecção de pontos destacados), criamos um grafo a partir do esqueleto, cujo processo de criação descrevemos a seguir.

\subsubsection{Construção do grafo para processamento do esqueleto}

A partir da imagem do esqueleto, criamos o grafo $G_{s}\left(V_{s}, E_{s}\right)$, no qual cada nó corresponde a um voxel, e cada aresta representa a conetividade entre os voxels dentro de uma 26-vizinhança. Adicionalmente, cada nó recebe como atributo as coordenadas do voxel a ele associado, e cada aresta recebe a distância euclidiana entre os nós conectados por ela. Esse processo é realizado pelo Algoritmo 1. Na Figura 4.3 e na Figura 4.4 são apresentados dois exemplos de grafos obtidos com o algoritmo em questão. 


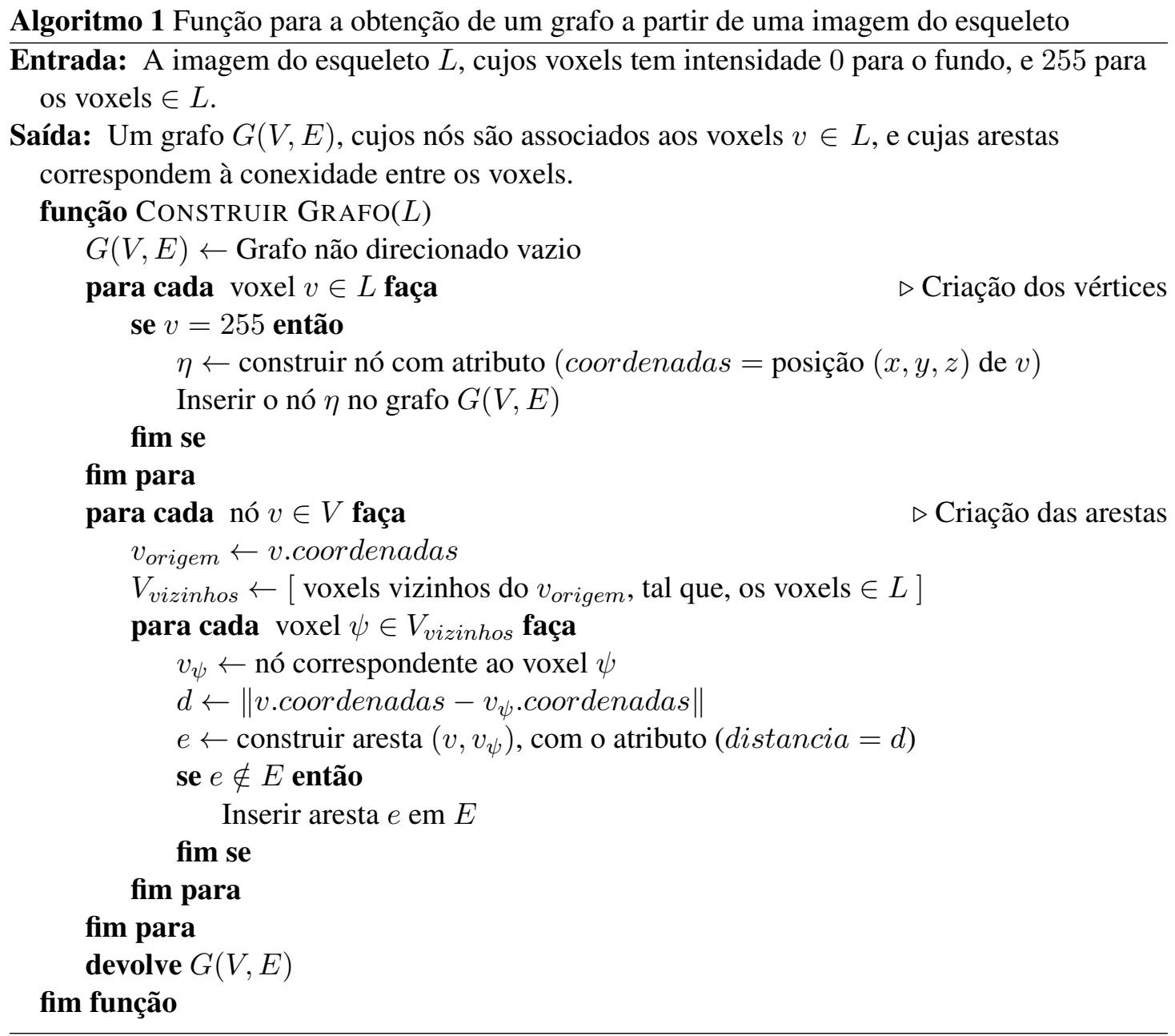

\subsubsection{Atribuição de identificadores de segmento}

Nessa etapa o grafo $G$ (criado anteriormente) é etiquetado de forma que cada segmento que possua mais do que $x$ voxels receba um identificador, o qual é usado posteriormente para a redução do número de falsos segmentos (ruido).

Para tanto, primeiro classificamos os vértices do grafo da seguinte forma:

Vértice terminal: Se o vértice $(v)$ tem grau 1 , ou seja $\operatorname{deg}(v)=1$.

Vértice de segmento: $\operatorname{Se} \operatorname{deg}(v)=2$.

Vértice de possível bifurcação: $\operatorname{Se} \operatorname{deg}(v) \geq 3$ 
Figura 4.3: Exemplo de grafo obtido a partir de um esqueleto, o peso das arestas é a distancia Euclidiana entre os voxels

(a)

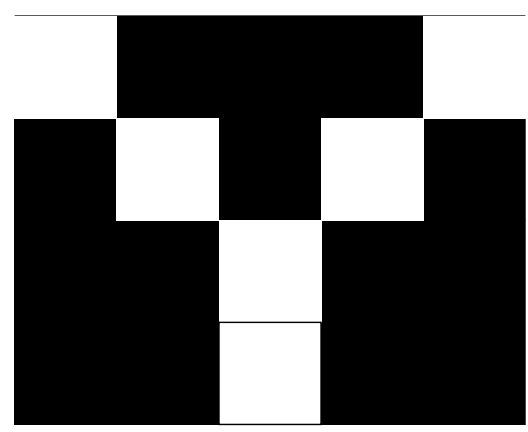

(b)

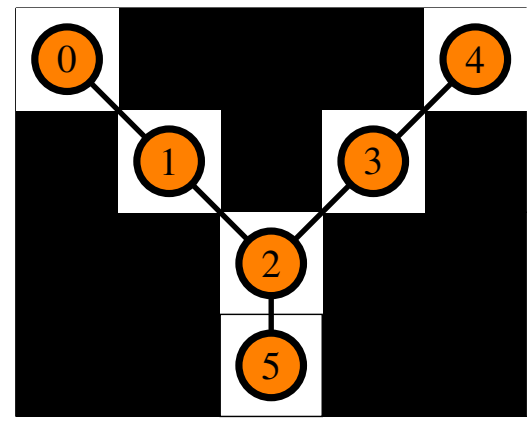

(c)

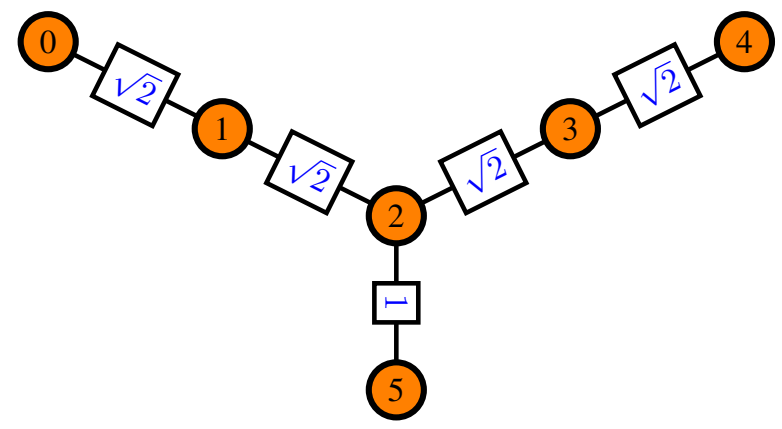

Podemos notar que os nós de grau $\geq 3$ não necessariamente correspondem a pontos de bifurcação. Por exemplo, na Figura 4.4 os nós 2, 3 e 5 têm grau 3, porém apenas um deles deveria ser considerado como ponto de bifurcação. Para resolver esse problema, executamos os seguintes passos: i) atribuir identificadores de segmento aos voxels que claramente não são bifurcações, e detectar as regiões de conflito, ii) solucionar as regiões de conflito escolhendo os caminhos mais curtos entre os segmentos vizinhos. Detalhamos nossa abordagem a seguir.

Paso 1: Cria-se um subgrafo $G^{\prime}$, formado por todos os vértices de segmento detectados até o momento, pelos vértices terminais em $G$, e por todas as arestas em $G$ existentes entre os vértices escolhidos, ou seja, criamos um grafo induzido $G^{\prime}$ em $G$ a partir dos vértices terminais e dos que apresentam grau igual a 2. Em seguida, atribui-se um identificador diferente para cada componente conexa $C \in G^{\prime}$, sempre que a componente tenha mais do que $x$ nós (i.e., $\|V(C)\|>$ $x$ ). Por fim, atribuímos um identificador diferente para cada componente e etiquetamos todos seus vértices com o respectivo identificador. 
Figura 4.4: Exemplos de grafos obtidos a partir de esqueletos, o peso das arestas é a distância euclidiana entre os voxels

(a) Exemplo de esqueleto em uma bifurcação

(b) Grafo obtido
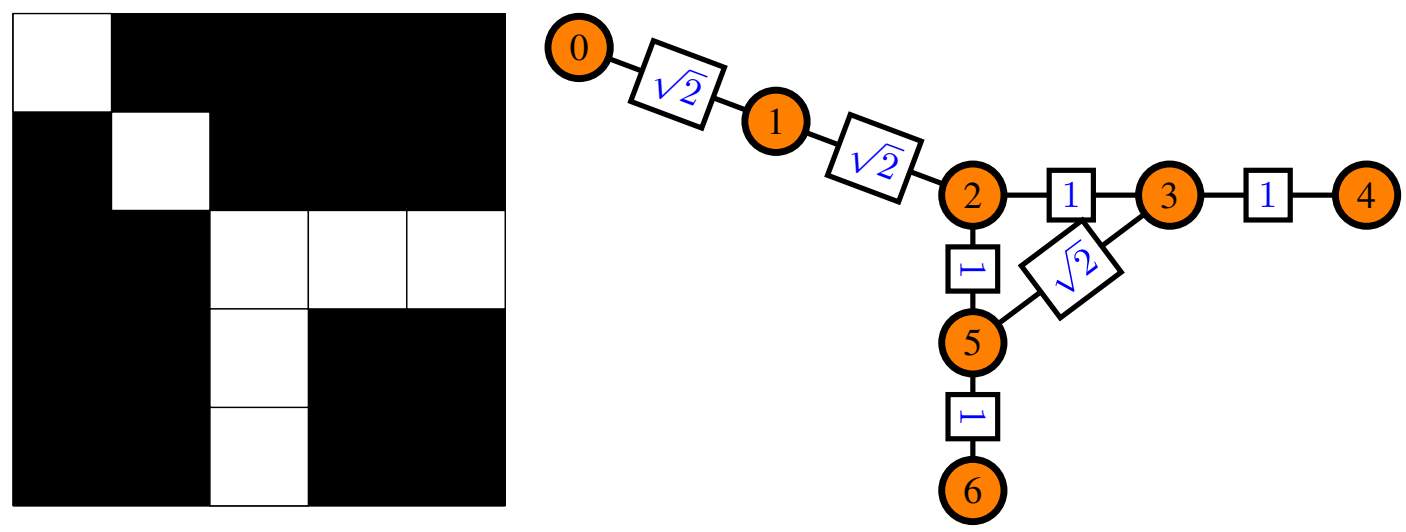

Todos os vértices de $G$ que não foram etiquetados são considerados como de possível bifurcação e são marcados com um identificador diferente. No nosso caso o identificador é " 0 ”. Na sequência, caso dois ou mais vértices adjacentes tenham sido etiquetadas com “ 0 " serão considerados como sendo uma região de conflito.

Dois exemplos simples da atribuição de identificadores são mostrados na Figura 4.5a. Nela, é mostrado um exemplo sem região de conflito, já o exemplo da Figura 4.5b mostra uma região de conflito formada por 3 vértices destacados em vermelho.

Figura 4.5: Atribuição de identificadores de segmento aos grafos mostrados na Figura 4.4. a) Grafo com 3 segmentos identificados em azul e um ponto de bifurcação de cor vermelha, $b) 3$ segmentos em azul e uma região de conflito com 3 nós vermelhos

(a)

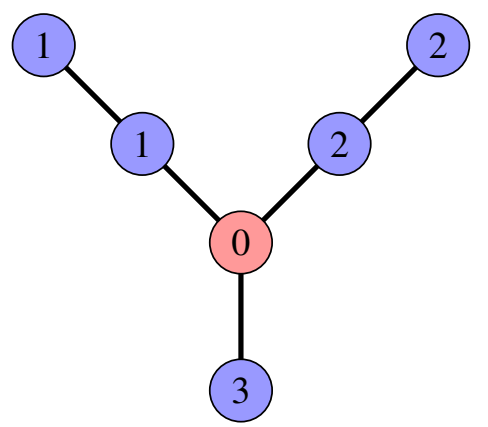

(b)

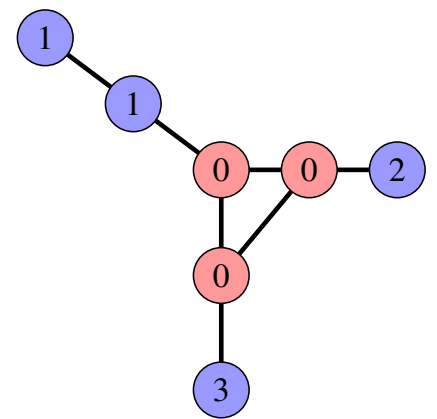




\subsubsection{Resolução das regiões de conflito}

Essa etapa tem como objetivo re-etiquetar os nós das regiões de conflito. As regiões de conflito são subgrafos conexos formados pelos vértices que ainda não foram classificados como parte de algum segmento. No processo, algumas arestas e até vértices excedentes poderão ser apagados do grafo original. A seguir incluímos duas definições que serão usadas na descrição de nossa abordagem.

Definição 1 Seja um grafo $G=(V, E)$ e um subgrafo $C=\left(V^{\prime}, E^{\prime}\right)$, com $C \subseteq G$, cujos vértices pertencem a uma região de conflito. Um vértice $v \in V \backslash V^{\prime}$ é adjacente à região de conflito $C$ se $\exists u \in V^{\prime}$, tal que, $\exists(u, v) \in E(G)$.

Definição 2 Seja um segmento $S=\left\{v_{0}, v_{1}, \ldots, v_{n}\right\}, v_{i} \in G$, o segmento $S$ é um segmento terminal se pelo menos um dos seus nós tem grau 1.

Nossa abordagem para a resolução de conflitos, tal como mostrado no Algoritmo 2, funciona da seguinte forma:

- Se uma região de conflito tem apenas dois nós adjacentes a ela, então calculamos o caminho mínimo entre os dois nós, e os nós que não formam parte do caminho obtido são apagados junto com suas respetivas arestas.

- Se a região tiver mais de dois nós adjacentes a ela, construímos uma árvore de espalhamento mínima, com os vértices da região e os nós adjacentes. Em seguida, as arestas da região de conflito que não formam parte da árvore são removidas. Além disso, são escolhidos os caminhos mínimos entre os nós adjacentes na árvore, e todo vértice e aresta da região de conflito que não forma parte desses caminhos são considerados desnecessários e, portanto, são também apagados.

- Depois de resolver as regiões de conflito, os vértices de todo o grafo são reclassificados e atribuímos um novo identificador de segmento para eles, já que após a correção de conflitos, os vértices com grau $>=3$ são sempre considerados como bifurcação. $O$ resultado do uso do algoritmo no grafo apresentado na Figura 4.5 é mostrado na Figura 4.6.

\subsubsection{Remoção de falsos segmentos}

O grafo obtido anteriormente pode conter falsos segmentos, possivelmente produzidos por erros no processo de esqueletização, em sua maioria consequências de bordas não suaves na seg- 


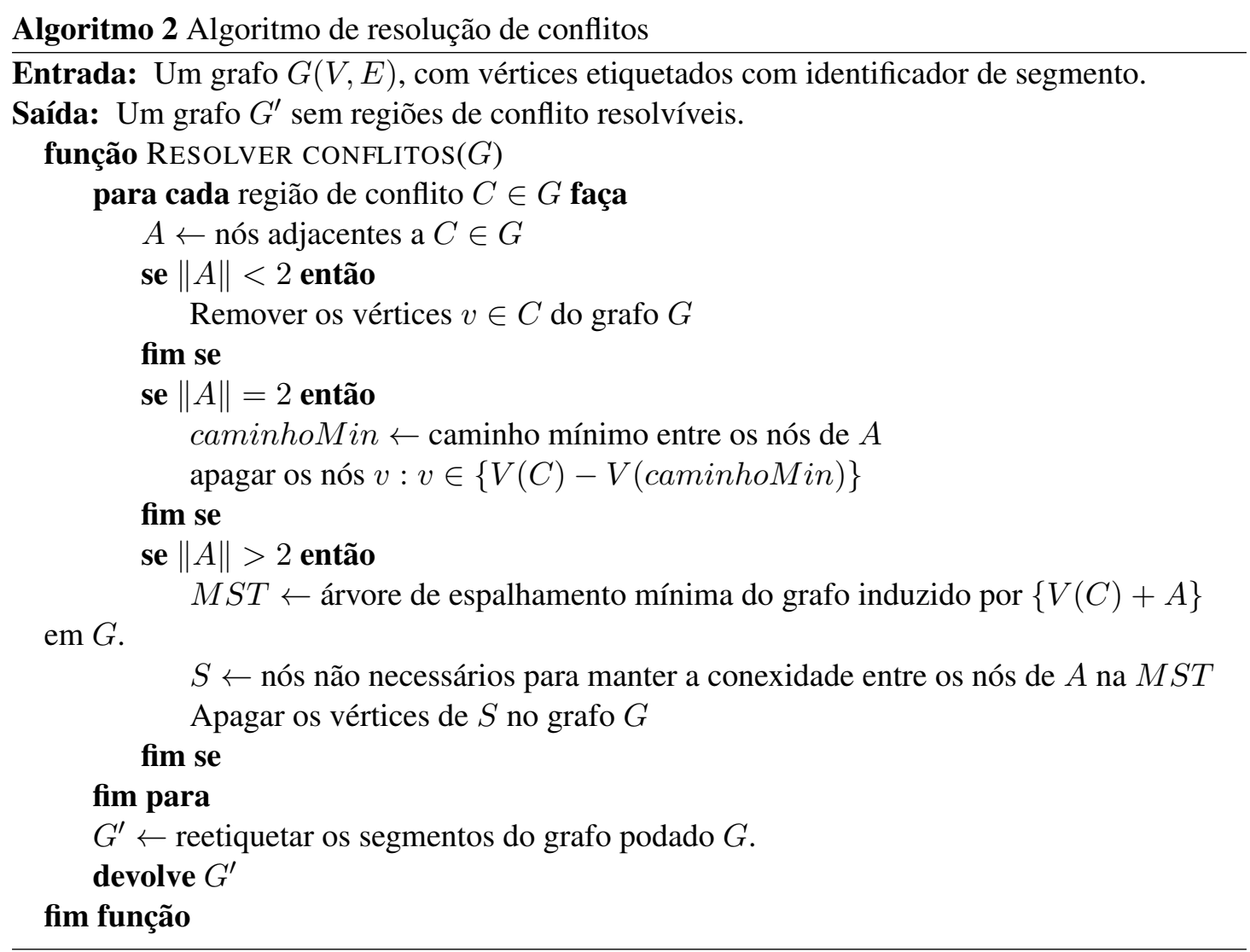

mentação. Para reduzir esse problema, escolhemos um tamanho de segmento mínimo $(x)$, e estabelecido este limiar, removemos os segmentos que apresentem tamanho menores do que este ${ }^{1}$, denominamos este processo poda ponderada, a qual, funciona da seguinte forma: se $\|S\|<x$ e $S$ é um segmento terminal, removemos todos os vértices de $S$ e as arestas conexas a eles. A poda continua até que não exista nenhum segmento com essas características.

A partir desse grafo começaremos o processo de análise e de extração da representação, para o qual, o primeiro passo é a detecção de pontos de controle.

\subsubsection{Determinação de pontos de controle}

Nossa representação tem que ser capaz de replicar a localização, tamanho e a tortuosidade dos segmentos vasculares. Estas características são apreciáveis também no esqueleto de cada segmento, pelo que, nesta etapa, consideramos cada segmento da rede vascular como uma curva

${ }^{1}$ Em nossos experimentos usamos um valor $x$ aproximadamente igual ao raio dos segmentos que queremos analisar. 
Figura 4.6: Exemplos de resolução de conflitos do grafo na Figura 4.4b.

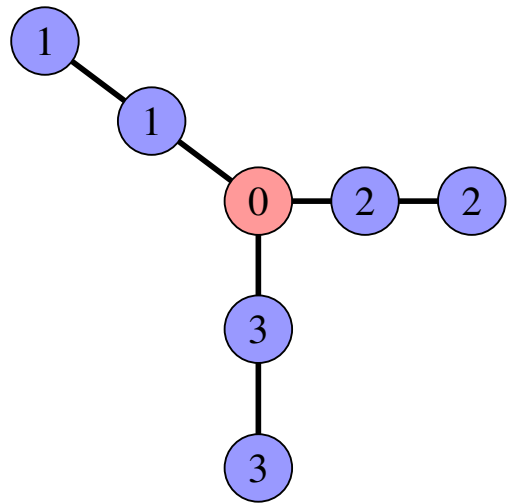

Figura 4.7: Exemplo de pontos de controle equidistantes que não representam a curva adequadamente.

(a) Curva exemplo e pontos de controle equidistantes

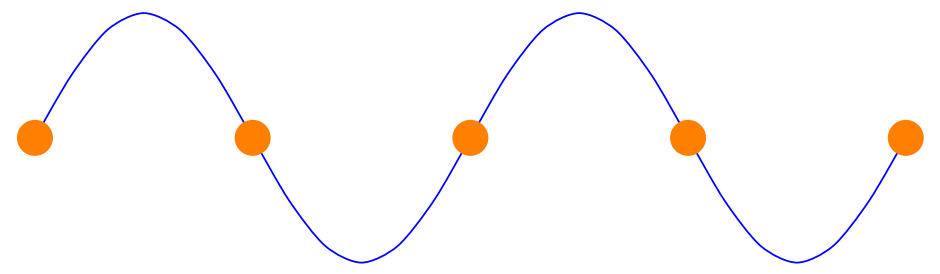

(b) Interpolação usando os pontos de controle obtidos em (a)

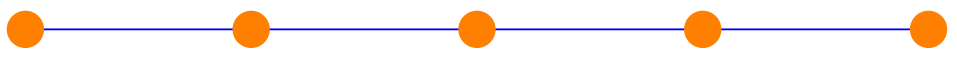

que passa por todos os voxels do esqueleto do segmento.

Uma forma de determinar uma curva é usarmos pontos de controle, os quais podem ser interpolados para obter outros pontos ao longo da curva. Da mesma forma, esse tipo de ponto pode ser usado tanto para caracterizar, como para reconstruir segmentos de redes vasculares. No entanto, deve-se ter o cuidado de escolher pontos adequados para essa tarefa de replicação, já que a escolha de poucos pontos ou pontos aglomerados em pequenas regiões pode levar à perda de informação.

Uma abordagem simples de escolha de pontos de controle é a seleção de $x$ pontos equidistantes na linha central do segmento. Entretanto, tal como mostrado na Figura 4.7, segmentos com muita tortuosidade poderiam não ser adequadamente representados, ao mesmo tempo, em segmentos retos a criação de muitos pontos poderia ser desnecessária.

Para atenuar os problemas anteriores deve-se escolher os pontos mais significativos da curva 
como pontos de controle, de maneira que estes possam determinar melhor a curva. Para tanto, desenvolvemos um método de obtenção de pontos de controle em curvas abertas, o qual é baseado no trabalho de Rahman et al. [2008] que propõe um algoritmo de identificação de pontos significativos em curvas fechadas.

Nossa abordagem, mostrada no Algoritmo 3, foca-se na detecção de pontos significativos em curvas abertas, e funciona da seguinte forma: o algoritmo cria uma linha reta entre o primeiro e o último ponto da curva, e identifica o ponto do segmento com a maior distância mínima em relação à linha. Se essa distância for maior do que um dado valor, que chamaremos de margem de erro permitido, esse será um ponto significativo. A partir desse ponto, a curva será dividida em duas curvas, e cada uma delas será analisada recursivamente.

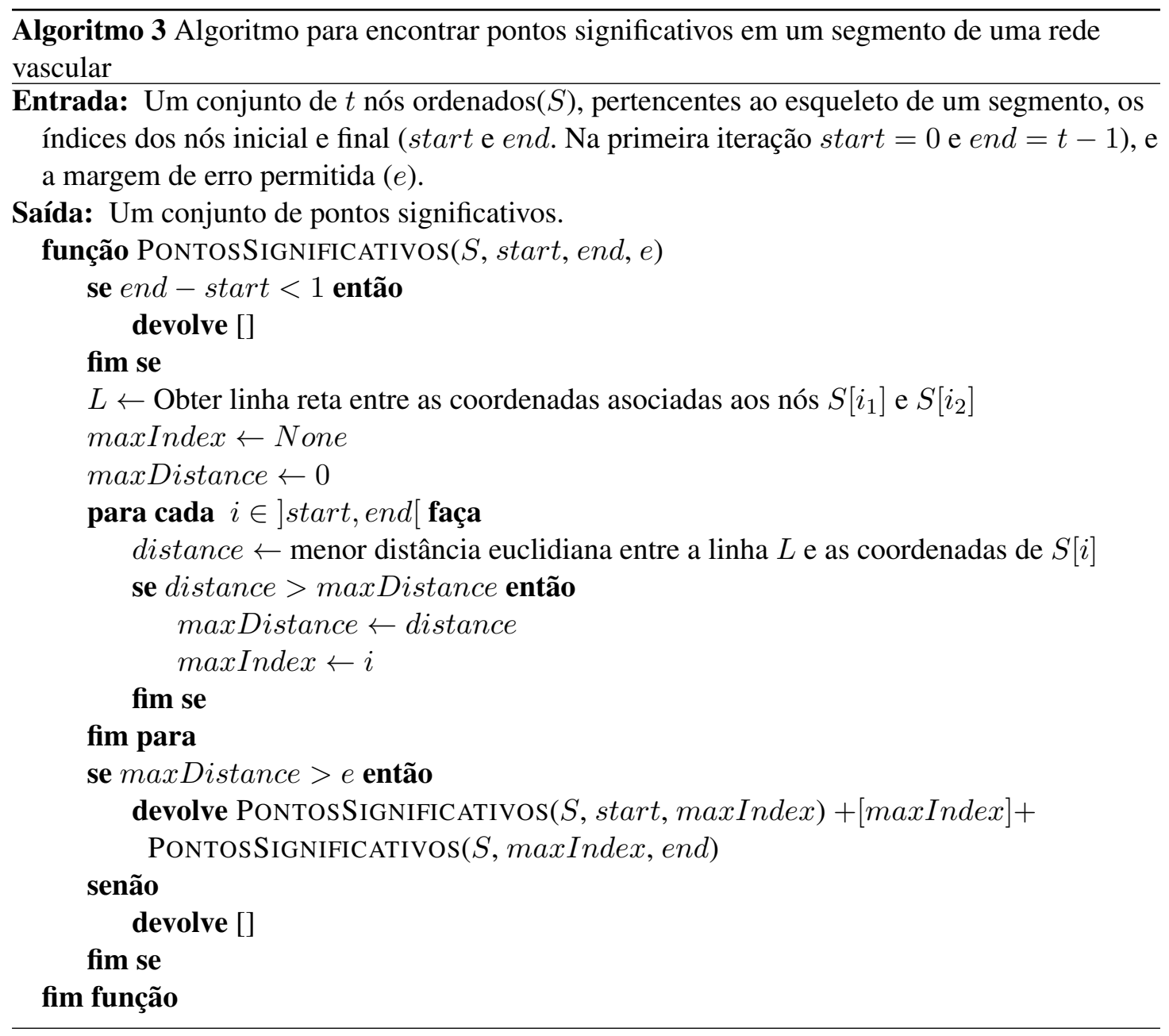

Uma simulação do algoritmo é mostrada na Figura 4.8. Nela é apreciada uma curva senoidal inicial e o resultado do algoritmo em 3 diferentes iterações. Essa abordagem evita os problemas 
descritos no uso de pontos equidistantes. Porém, dado que usamos $B$-splines cúbicas como método de interpolação, segmentos que intercalem regiões com alta curvatura e regiões retas podem resultar em curvas diferentes das originais, tal como o exemplo mostrado na Figura 4.9.

Figura 4.8: Simulação do algoritmo de escolha de pontos significativos

(a) Curva inicial

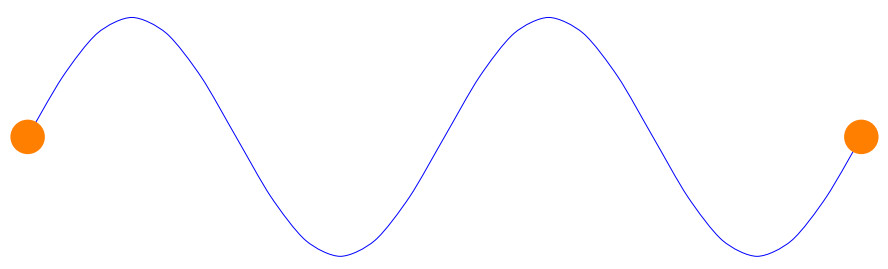

(b) Primeira iteração, o primeiro ponto mais distante à curva é escolhido

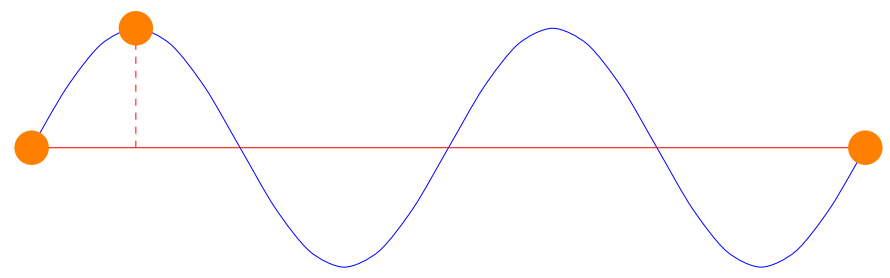

(c) Segunda iteração

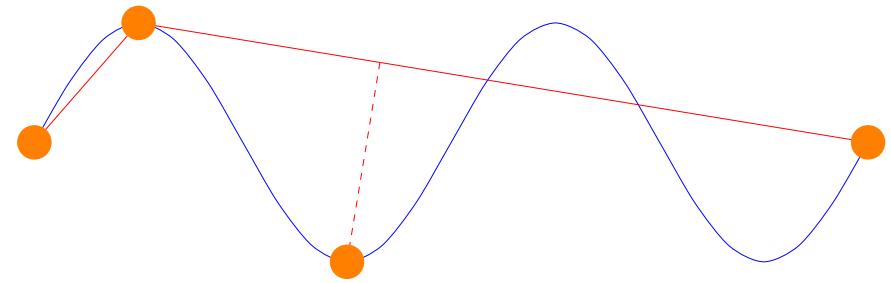

(d) Pontos significativos na última etapa

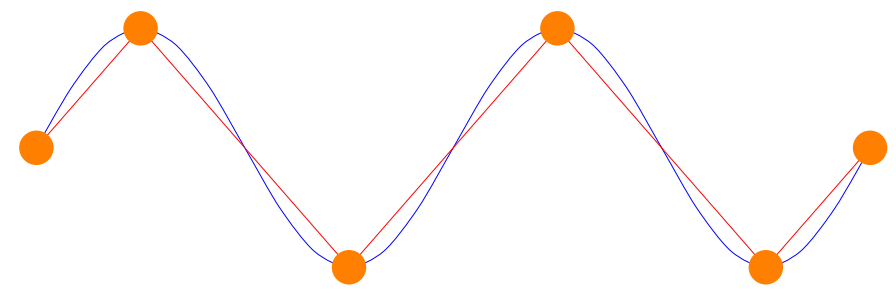

Esse problema é resolvido incluindo uma etapa de refinamento de curvas ao Algoritmo 3. Nessa etapa, usamos os pontos significativos obtidos pelo método de Rahman et al. [2008] e os interpolamos. Essa curva interpolada $\mathscr{C}^{\prime}$ é dividida em regiões, cada uma das quais é delimitada por um dos pontos de controle usados na sua geração. Finalmente, em cada região buscamos o 
Figura 4.9: Exemplo de pontos significativos obtidos com o Algoritmo 3 que não representam adequadamente à curva

(a) Curva inicial

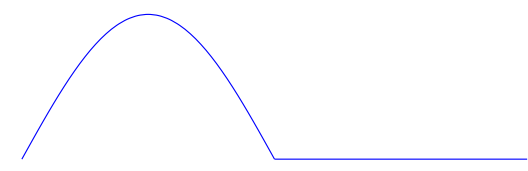

(b) Pontos significativos obtidos

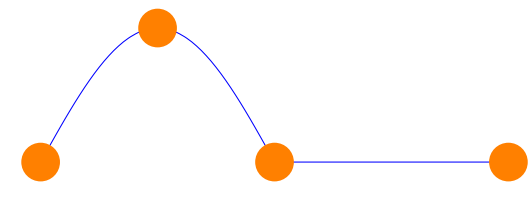

(c) Interpolação dos pontos obtidos

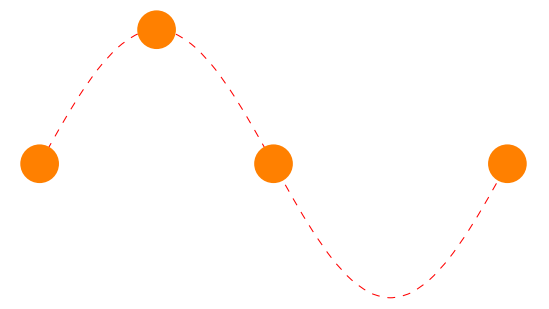

ponto $P \in \mathscr{C}^{\prime}$ com a maior distância mínima à curva original $\mathscr{C}$, ou seja:

$$
P=\text { PontoMaiorDistânciaMínima }\left(\mathscr{C}, \mathscr{C}^{\prime}\right)=\underset{P^{\prime} \in \mathscr{C}^{\prime}}{\operatorname{argmax}}\left(\operatorname{DistânciaMínima}\left(P^{\prime}, \mathscr{C}\right)\right),
$$

onde DistânciaMínima $(P, C)$ é a menor distância de um ponto $P$ a uma curva $C$, e é dado por:

$$
\operatorname{DistânciaMínima}(P, C)=\underset{P^{\prime} \in C}{\operatorname{minimize}}\left|P^{\prime}-P\right| \text {. }
$$

Uma vez obtidos os pontos $P_{i}$ com maior erro em cada região, se a distância DistânciaMínima $\left(P_{i}, \mathscr{C}\right)$ for maior ao erro permitido, consideramos $P_{i}$ como sendo um novo ponto de controle. Após analisar todas as regiões, o processo pode ser repetido um dado número de vezes ou até atingir a estabilidade, que acontece quando durante uma iteração nenhum novo ponto de controle é adicionado. Todo esse procedimento é apresentado no Algoritmo 4. 


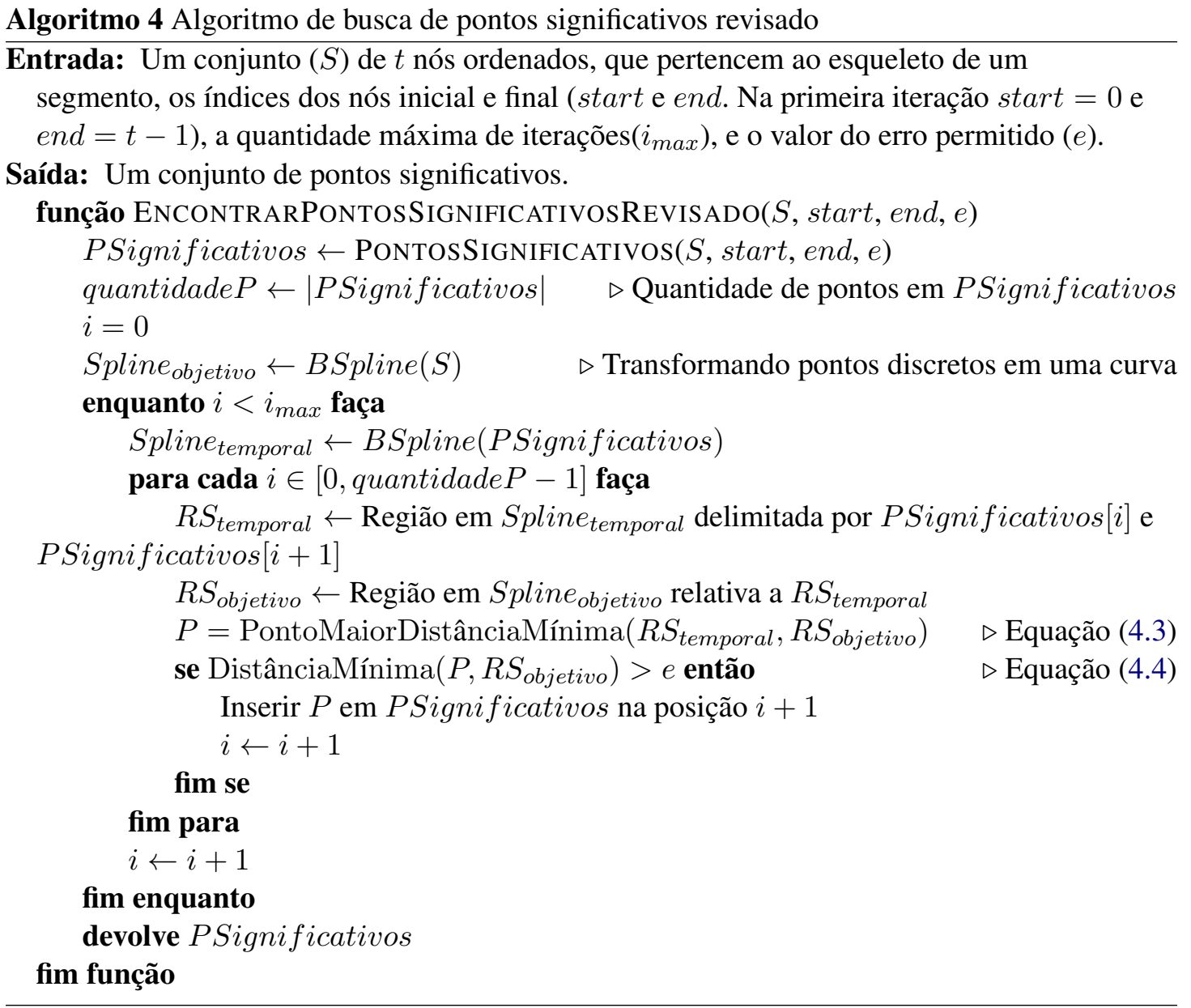

\subsubsection{Geração da representação em cadeia de texto}

Nas seções anteriores descrevemos nosso processo de esqueletização e o processo de detecção de pontos destacados. Nesta fase, o esqueleto encontra-se armazenado em uma estrutura de grafos, na qual foram devidamente identificados os nós escolhidos como pontos de controle, sendo que cada um dos nós possui como atributo seu raio e um identificador de segmento.

A partir dessa informação, nesta seção, descrevemos o processo de geração de uma representação textual, que é equivalente ao procedimento inverso de geração de imagens desenvolvido por Valverde [2012]. Neste trabalho usa-se um conjunto de símbolos que foi posteriormente estendido em [Valverde et al., 2013]. Na presente metodologia, usamos esses símbolos com algumas melhoras, tanto em sua interpretação como na quantidade de parâmetros que aceitam. Estas mudanças possibilitam tanto a representação de estrutura de grafos (já que [Valverde, 2012; 
Valverde et al., 2013] foca apenas em árvores), como a simplificação do processo de quantificação que descreveremos posteriormente.

Inicialmente precisamos definir alguns valores: um ponto inicial, um vetor de direção inicial $\left(\vec{V}_{\text {direção }}\right)$ e um vetor perpendicular ao anterior $\left(\vec{V}_{\text {perpendicular }}\right)$. A atribuição de diferentes valores aos vetores $\vec{V}_{\text {direção }}$ e $\vec{V}_{\text {perpendicular altera apenas os ângulos de rotação inicial, não tendo }}$ impacto no processo de quantificação posterior desde que esses mesmos valores sejam usados no processo em questão. A fim de padronizar os resultados, usamos $\vec{V}_{\text {direção }}=(0,-1,0)$ e $\vec{V}_{\text {perpendicular }}=(0,0,1)$ como valores padrões. Para definir as coordenadas do ponto inicial, escolhemos um voxel e calculamos suas coordenadas de objeto, tal como descrito na Seção 3.1.1. Em nossos experimentos, escolhemos o voxel correspondente ao ponto terminal com o maior raio.

Uma vez definidas as variáveis iniciais, começamos o traçamento da rede vascular, no qual percorremos os vasos segmento por segmento e extraímos a representação textual de cada um deles.

\subsubsection{Representação de um segmento}

Para extrair a representação de uma rede vascular, primeiramente analisamos e extraímos a representação de cada um dos segmentos. Para tanto, dividimos o segmento em um conjunto de subsegmentos retos, o que é feito por meio do uso de pontos de controle previamente calculados, de maneira que estes determinem o começo e o fim de cada subsegmento. Essas subdivisões do segmento permitem-nos simular a tortuosidade e curvatura dos vasos.

Os subsegmentos podem ser modelados por meio do seu vetor de direção, seu comprimento, e seu raio; para isso, usamos o $\vec{V}_{\text {direção, anteriormente definido. Dado que os subsegmentos }}$ (dentro de um mesmo segmento) são sequencialmente conexos, é preciso rotacionar $\vec{V}_{\text {direção }}$ antes da criação de cada subsegmento. Assim, nossa representação registra a criação de cada um desses subsegmentos e a sequencia de rotações necessárias. Essas ações são representadas pelos seguintes símbolos:

- Símbolos de início e fim de um segmento: $\{(\boldsymbol{i d})$,$\} . Estes símbolos determinam o começo$ e o fim de um segmento, o qual pode ser composto por um ou mais subsegmentos. Este símbolo aceita um parâmetro de identificação do segmento.

- Símbolo de criação de subsegmentos retos: $f$ (comprimento, diâmetro). Símbolo usado para criar subsegmentos, o qual requer dois parâmetros: o comprimento e o diâmetro. O 
parâmetro comprimento é calculado como sendo a distância euclideana entre as coordenadas do voxel inicial do subsegmento $\left(X_{i}\right)$ e do voxel final $\left(X_{f}\right)$. O parâmetro diâmetro é a média entre os diâmetros ao longo do subsegmento. Ao finalizar a criação do subsegmento, o valor da variável ponto inicial é atualizado com sua coordenada final.

- Símbolos de rotação do plano de rotação: /(graus), *(graus). Esses símbolos são criados sempre que o plano de rotação atual impossibilite a criação de um subsegmento no ponto desejado. $\mathrm{O}$ parâmetro requerido é igual ao ângulo necessário para rotacionar o plano de rotação de forma que $X_{f}, X_{i}$, e $\left(X_{i}+\vec{V}_{\text {direção }}\right)$ fiquem num mesmo plano. Adicionalmente, o símbolo “”” faz rotações no sentido horário, e o símbolo “*” no sentido anti-horário.

- Símbolos de rotação no plano atual: +(graus), -(graus). Usado para rotar $\vec{V}_{\text {direção }}$ no sentido horário e anti-horário, respectivamente. O parâmetro graus, requerido por essa instrução, é o ângulo entre $\vec{V}_{\text {direção }}$ e $\vec{X}_{f}-X_{i}$.

Com base nesses símbolos, extraímos a representação de um segmento $S$, formado pelos pontos $p_{0}, p_{1}, \cdots, p_{m}$, como descrito a seguir: sejam os pontos de controle $P_{0}, P_{1}, \cdots, P_{n} \in S$ de um dado segmento. Começamos o processo de rastreamento desde $P_{0}$, e o conectamos através de um subsegmento com o próximo ponto de controle $\left(P_{1}\right)$. Para tanto, primeiro é necessário rotacionar $\vec{V}_{\text {perpendicular }}$ em $\alpha$ graus, com o fim de mudar o plano de rotação de $\vec{V}_{\text {direção}}$; na sequência, rotaciona-se em $\beta$ graus o $\vec{V}_{\text {direção }}$; posteriormente cria-se o subsegmento com comprimento $(c)$ e raio $(r)$. As fórmulas do cálculo de $\alpha, \beta, c$ e $r$ são descritas a seguir:

$$
\alpha=\arccos \left(\vec{V}_{\text {perpendicular }} \cdot\left(\vec{V}_{\text {direção }} \times\left(P_{i+1}-P_{i}\right)\right)\right),
$$

se a rotação é no sentido horário em relação a $\vec{V}_{\text {direção, incluímos nas instruções a instrução }}$ “/( $\alpha)$ ”, caso contrário “* $*(\alpha)$ ”.

$$
\beta=\arccos \left(\vec{V}_{\text {direção }} \cdot\left(P_{i+1}-P_{i}\right)\right)
$$

se a rotação é no sentido horário em relação a $\vec{V}_{\text {perpendicular }}$, incluímos nas instruções a instrução “- $-(\beta)$ ”, caso contrário “ $+(\beta)$ ”.

Finalmente, os parâmetros de comprimento $c$ e raio $r$ são obitos por:

$$
c=\left\|P_{1}-P_{0}\right\|
$$




$$
r=\frac{1}{m} * \sum_{j=\operatorname{index}\left(P_{i}\right)}^{\operatorname{index}\left(P_{i}+1\right)} \operatorname{raio}\left(p_{j}\right),
$$

onde index $\left(P_{i}\right)$ é a posição do ponto de controle $P_{i}$ no segmento, e raio $\left(p_{j}\right)$ é o valor do raio do segmento no ponto $p_{j}$.

Figura 4.10: Extração da representação de um segmento, na qual $a_{i}$ são ângulos, $c_{i}$ comprimentos e $d_{i}$ diâmetros. (a) Segmento original, (b) Pontos de controle no esqueleto do segmento, (c) sequência de instruções para cada rotação e criação de sub-segmentos, $e(d)$ Representação textual do segmento

(a)

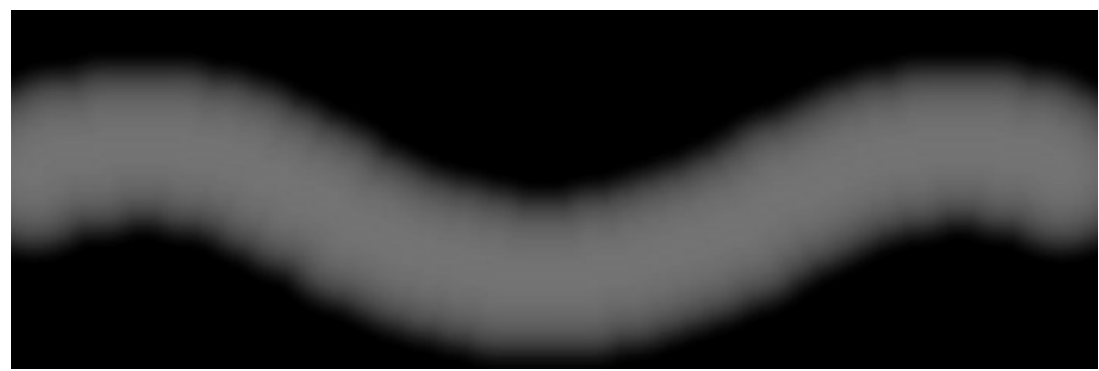

(b)

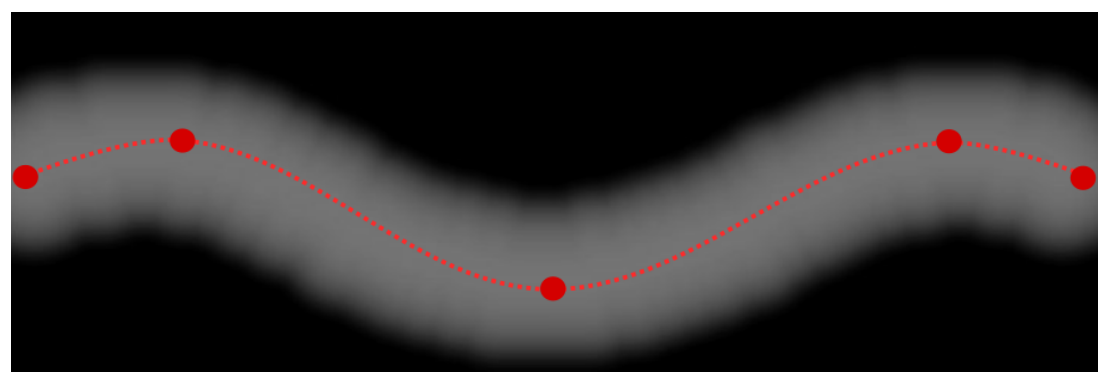

(c)

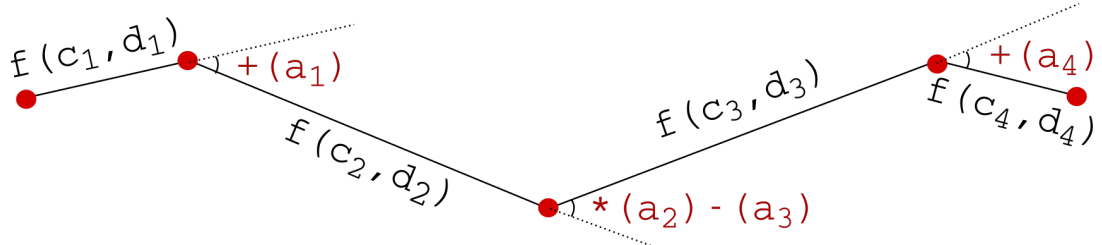

(d)

$$
\left\{(i d)\left(\mathbf{f}\left(c_{1}, d_{1}\right)+\left(a_{1}\right) \mathbf{f}\left(c_{2}, d_{2}\right) *\left(a_{2}\right)-\left(a_{3}\right) \mathbf{f}\left(c_{3}, d_{3}\right)+\left(a_{4}\right) \mathbf{f}\left(c_{4}, d_{4}\right)\right\}\right.
$$

Na Figura 4.10 é mostrado um exemplo do nosso processo de representação, obtido a partir 
de um segmento. No exemplo usamos parâmetros genéricos, os quais são simbolizados com: $c_{i}$ para os comprimentos, $d_{i}$ para os diâmetros, $a_{i}$ para os ângulos, e $(i d)$ para o identificador do segmento.

\subsubsection{Estrutura da rede vascular}

As redes vasculares são usualmente representadas com estruturas de árvores, porém é comum existirem ciclos na vasculatura, tanto nas escalas menores (capilares), quanto nas grandes escalas, nas quais podemos encontrar estruturas de segmentos maiores. Uma das mais conhecidas estruturas vasculares, e que possui ciclos, encontra-se na base do cérebro e é chamada de CoW. Por este motivo, é necessário possibilitar esse tipo de estruturas em nossa representação. Para tanto, incluímos os pontos de bifurcação na representação, o que torna possível replicar a conectividade dos segmentos. Nesse processo usamos os seguintes símbolos:

- Símbolo de bifurcação: [(id). O símbolo requer um parâmetro que representa um identificador. Se não existe uma bifurcação com o identificador correspondente, então é criado um ponto de bifurcação com o identificador de segmento. Porém, se a bifurcação existe, temos que considerar que o ponto atual corresponde a um ponto de bifurcação anteriormente definido. Desta forma, o símbolo é útil para determinar a existência de ciclos na rede vascular e simplifica o processo de quantificação.

- Retornar a um ponto de bifurcação: J(id). Recupera os dados de um ponto de bifurcação previamente armazenado com o identificador dado. Nos segmentos folha não é necessário armazenar os pontos já que não serão mais usados.

Essas instruções tornam possível a representação tanto de estruturas acíclicas, como a mostrada na Figura 4.11, quanto de estruturas que contém ciclos, como a mostrada na Figura 4.12. Nestes exemplos, $S_{i}$ é a representação textual do segmento com identificador $i$, obtido na etapa anterior. 
Figura 4.11: Representação de uma rede vascular em forma de árvore

(a) Estrutura em forma de árvore

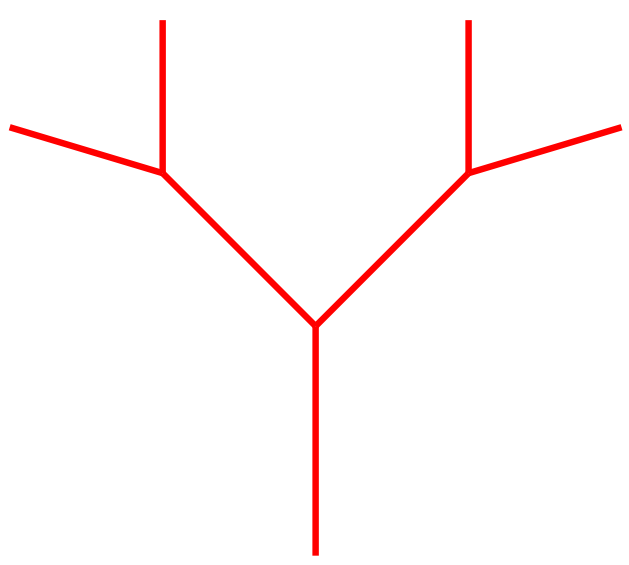

(b) Segmentos $\left(S_{i}\right)$ e seus delimitadores devidamente etiquetados em cor azul.

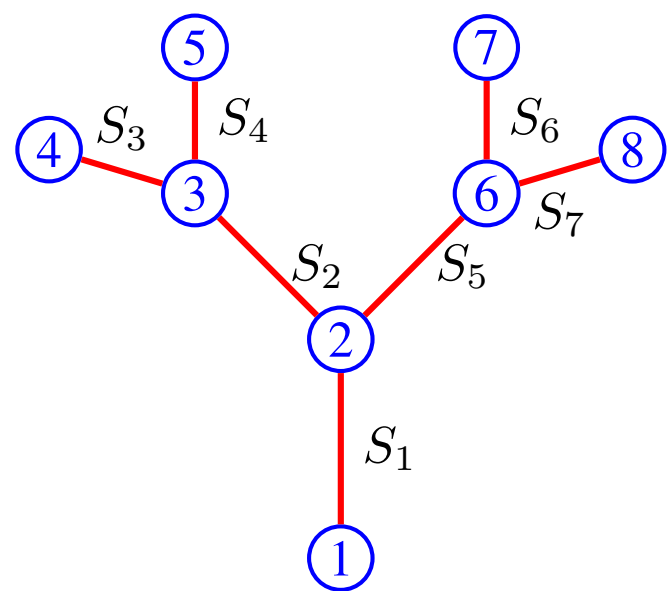

(c) Representação textual da estrutura mostrada, onde $S_{i}$ é a representação textual do $i$-ésimo segmento

$$
\text { [(1) } S_{1}\left[(2) S_{2}\left[(3) S_{3}\right](3) S_{4}\right](2) S_{5}\left[(6) S_{6}\right](6) S_{7}
$$

Figura 4.12: Representação de uma estrutura cíclica

(a) Estrutura inicial

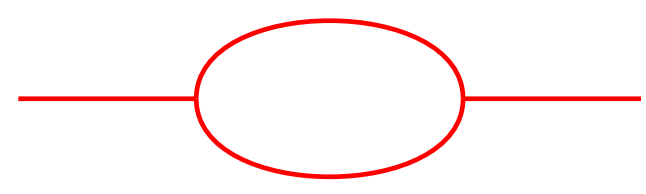

(b) Segmentos $\left(S_{i}\right)$ e seus delimitadores devidamente etiquetados em cor azul.

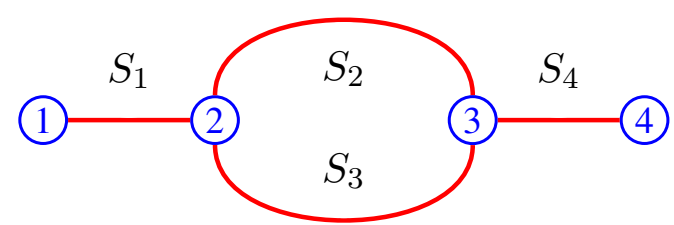

(c) Representação textual da estrutura mostrada, onde $S_{i}$ é a representação textual do $i$-ésimo segmento

$$
\left[( 1 ) S _ { 1 } \left[( 2 ) S _ { 2 } \left[(3) S_{3}[(2)](3) S_{4}\right.\right.\right.
$$




\subsubsection{Armazenamento}

A cadeia gerada pode ser facilmente armazenada em um arquivo de texto, sendo que nele também devem ser incluídos os valores do ponto inicial, $\vec{V}_{\text {direção }}$, e $\vec{V}_{\text {perpendicular }}$, caso tenham sido usados valores diferentes aos valores padrão. Com o intuito de manter o mesmo padrão apresentado nos trabalhos de [Valverde, 2012; Valverde et al., 2013] armazenamos a representação no formato mostrado na fig. 4.13 .

Figura 4.13: Formato de armazenamento da representação textual e variáveis iniciais

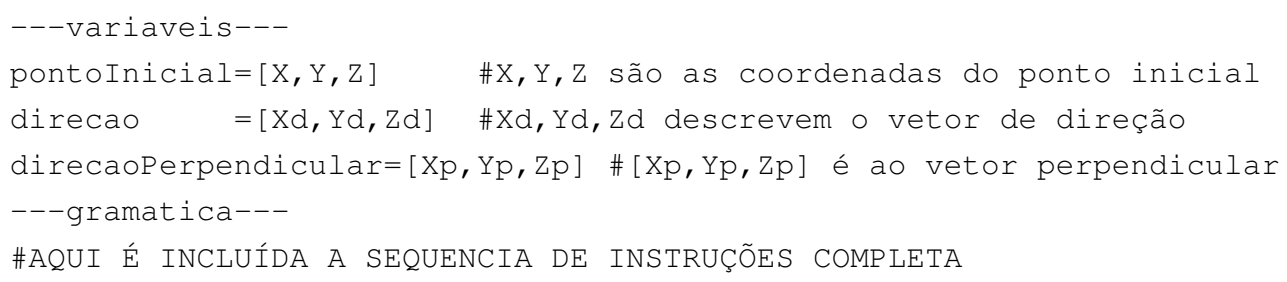

\subsubsection{Reconstrução}

Nossa representação é baseada nos trabalhos de geração de imagens sintéticas de [Valverde, 2012; Valverde et al., 2013], portanto a partir de nossa representação é possível criar imagens volumétricas com intensidades similares às encontradas em imagens de angio-RM ou angio-TC. No entanto, a metodologia de geração teve que ser adaptada e implementada para a viabilização de mudanças nas instruções, conforme descrito anteriormente.

O fato de poder reconstruir a imagem a partir da sua representação, mostra que por meio de nossa representação é também possível extrair quaisquer características, que possam ser obtidas da imagem segmentada original. Caso não se disponha de um método de extração de uma determinada característica por meio de nossa representação é possível reconstruir a imagem e extrair a característica desejada a partir dela.

\subsection{Quantificação}

O processo de quantificação de redes vasculares a partir de imagens é bem complexo, e usualmente está focado apenas na extração de determinadas características. Contudo, a partir da nossa representação é possível extrair uma ampla diversidade de características sem a necessidade de reanalisar a imagem original; mais especificamente, é possível obter todas as caracte- 
rísticas relativas à morfologia vascular que se mencionam na literatura e que foram revisadas no Capítulo 2.

Nosso processo de quantificação requer como entrada a representação da rede vascular em cadeia de texto. A partir da cadeia obtemos um grafo, no qual cada aresta é associada a um segmento da rede vascular, e cada vértice a um ponto terminal ou de bifurcação. Assim, para a obtenção de determinadas características, a execução da cadeia de instruções da representação pode ou não ser necessária. Por exemplo, para se obter a quantidade de bifurcações existentes numa rede vascular não é necessário reconstruir todos os segmentos da rede (o que implicaria executar a cadeia de instruções completa), basta fazer uma contagem dos vértices do grafo, tal como descrito nas próximas seções. Contudo, algumas características requerem a reconstrução de parte ou da totalidade dos segmentos, tais como as coordenadas de inicio, fim, ou direção de cada segmento.

$\mathrm{Na}$ sequência, descrevemos métodos de extração de características propostas a partir de nossa representação. As características selecionadas foram escolhidas por serem algumas das mais recorrentes na literatura.

\subsubsection{Construção do grafo de representação}

Quantificar redes vasculares por meio da nossa representação textual requer a construção de um grafo $G=(V, E)$, com vértices $V$ e arestas $E$, o qual deve possuir as seguintes características:

- Cada ponto terminal e de bifurcação no esqueleto é associado a um nó $n \in V$.

- Consideramos um segmento de rede vascular como a região limitada por bifurcações ou pontos terminais. Dessa forma, cada segmento da rede vascular é associada a uma aresta $e \in E$.

- Em cada aresta é armazenada a representação textual do segmento a ela associado.

Um exemplo de uma estrutura vascular similar à formada pelas artérias que conformam o CoW, bem como seu grafo resultante são mostrados na Figura 4.14. Nessa estrutura vascular assumimos que $\{a, b, c, \cdots\}$ são as representações textuais do segmento correspondente. 
Figura 4.14: Grafo representativo de uma estrutura vascular

(a) Estrutura de uma rede similar à do círculo de Willis

(b) Grafo representativo
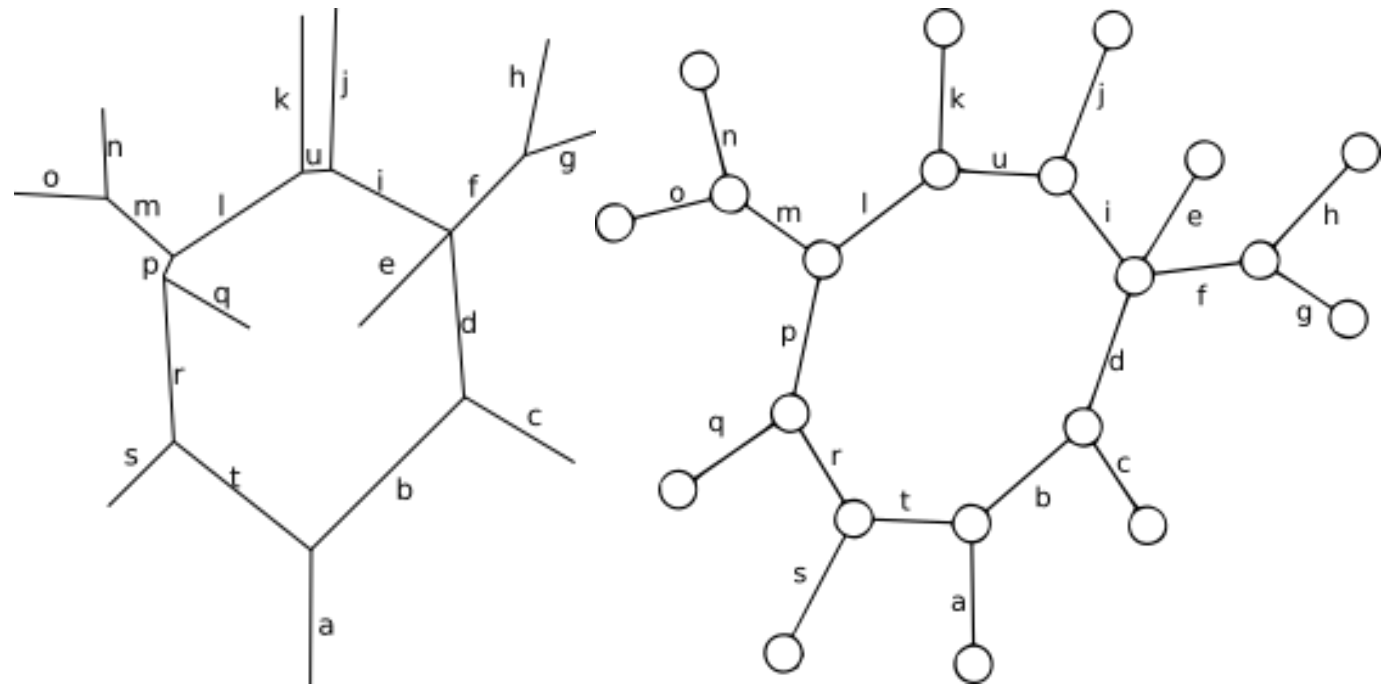

\subsubsection{Extração de características}

A partir dos trabalhos correlatos analisados e mostrados no Capítulo 2, escolhemos algumas das características mais usadas na literatura, e desenvolvemos métodos para extraí-las a partir da nossa representação. As características escolhidas foram descritas na Seção 3.6.1 e são listadas a seguir:

- Quantidade total de bifurcações.

- Quantidade de pontos terminais.

- Quantidade de segmentos.

- Comprimento dos segmentos.

- Raio médio dos segmentos.

- Comprimento total da representação.

- Níveis de bifurcação.

- Métrica de contagem de pontos de inflexão (Inflection Counting Metric, ICM).

- Métrica de distância (Distance Metric, DM). 
- $\mathrm{DM} \times \mathrm{ICM}$, tal como sugerido por Bullitt et al. [2003].

- Métrica de soma de ângulos (Sum Of Angles Metric, SOAM).

- Ordem de Strahler.

- Taxonomia de Tokunaga e sua matriz de proporção.

\subsubsection{Quantidade de elementos}

Características básicas das redes vasculares (tais como, quantidades de bifurcações, segmentos e pontos terminais) podem ser facilmente extraídas a partir do grafo $G=(V, E)$, construído anteriormente, tal como mostrado a seguir:

A quantidade de segmentos é igual à quantidade de arestas no grafo, ou seja $\|E(G)\|$.

A quantidade de bifurcações é igual a $\left\|V^{\prime}\right\|$, onde $V^{\prime}=\{v \in V(G) \mid \operatorname{grau}(v) \geq 2\}$, ou seja, é igual à quantidade de vértices de grau maior ou igual a 2.

A quantidade de pontos terminais é igual a $\left\|V^{\prime \prime}\right\|$, onde $V^{\prime \prime}=\{v \in V(G) \mid \operatorname{grau}(v)=$ $1\}$.

\subsubsection{Comprimento, raio e outros atributos dos segmentos}

Cada uma das arestas do grafo contém a cadeia de instruções de um segmento, cadeia que pode conter instruções para a criação de subsegmentos, com suas respectivas rotações. Isso torna possível obter os pontos mais representativos do segmento e seus respectivos raios.

A partir desses pontos de controle é calculada uma curva B-spline, a qual é usada para interpolar novos pontos, tal como mostrado na Figura 4.15. Finalmente, para um segmento $S$, cujos pontos de controle são $P_{1}, P_{2}, \ldots, P_{m}$, e cujos pontos interpolados são $p_{1}, p_{2}, \cdots, p_{n}$, calculamos o comprimento da seguinte forma:

$$
\operatorname{comprimento}(S)=\sum_{i=1}^{n-1}\left\|p_{i+1}-p_{i}\right\|
$$

$$
\text { raio } \operatorname{DoSegmento}(S)=\frac{1}{\operatorname{comprimento}(S)} * \sum_{i=1}^{n-1} \operatorname{raio}\left(p_{i}\right) *\left\|p_{i+1}-p_{i}\right\|
$$


Em nossos experimentos, calculamos um total de 50 pontos interpolados por segmento, uma vez que, valores maiores que este não alteraram os dados a serem quantificados de forma relevante.

Figura 4.15: Dados usados para o cálculo da DM.

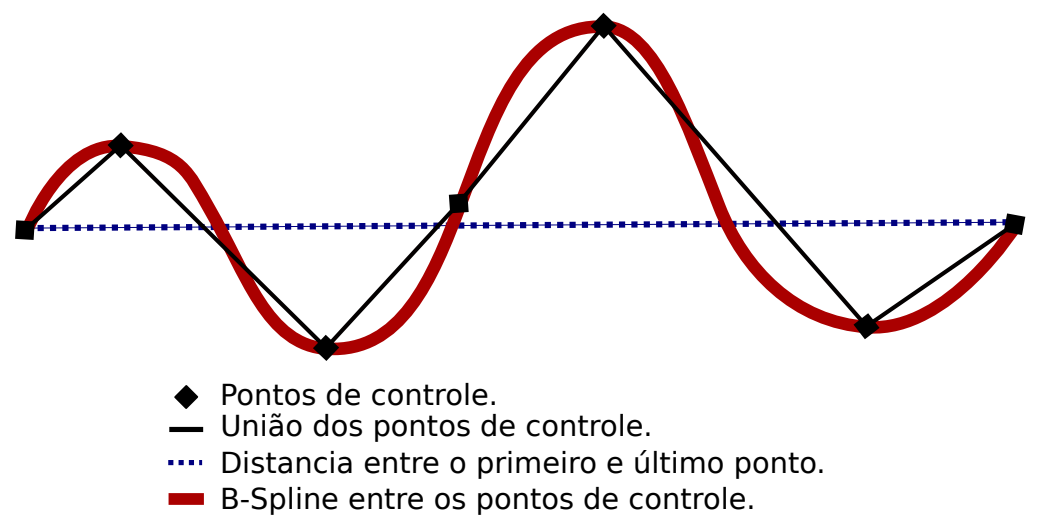

\subsubsection{Métrica de distância (Distance Metric, DM)}

A obtenção dessa métrica a partir da representação textual é feita para cada segmento da rede vascular. Para tanto, calculamos o comprimento do segmento $(S)$ (como mostrado na Equação (4.9)) e o dividimos pela distância Euclideana entre o ponto inicial $\left(p_{0}\right)$ e o ponto final $\left(p_{n}\right)$ do segmento. Ou seja:

$$
D M(S)=\frac{\text { comprimento }(S)}{\left\|p_{n}-p_{0}\right\|}
$$

\subsubsection{Métrica de contagem de pontos de inflexão (Inflection Counting Metric, ICM)}

Calculamos um triedro de Serret-Frennet (visto na Seção 3.6.1.2) para poder detectar os pontos de inflexão do segmento analisado. Consideramos que um ponto é de inflexão quando o vetor normal $(N)$ rotaciona em um ângulo de $\approx 180^{\circ}$. Para tanto, tal como sugerido por Bullitt et al. [2003], calculamos os vetores do triedro em cada ponto de controle do segmento da seguinte forma: Dados 3 pontos $P_{k-1}, P_{k}, P_{k+1}$, o vetor tangente do ponto $P_{k}$ é calculado com base no anterior e no próximo ponto $\left(P_{k-1}\right.$ e $\left.P_{k+1}\right)$ :

$$
\vec{T}=\frac{P_{k+1}-P_{k-1}}{\left\|P_{k+1}-P_{k-1}\right\|}
$$


O vetor normal é calculado como sendo o vetor unitário normal ao plano formado pelo vetor tangente atual e o vetor tangente do ponto anterior.

$$
\vec{N}=\frac{T_{P_{k}} \times T_{P_{k-1}}}{\left\|T_{P_{k}} \times T_{P_{k-1}}\right\|}
$$

\subsubsection{Métrica de soma de ângulos (Sum Of Angles Metric, SOAM)}

A SOAM é uma medida cujo valor é diretamente proporcional à tortuosidade de um segmento. Ela é calculada a partir de pontos discretos no segmento, razão pela qual usamos pontos interpolados pelo $B$-spline como base para o cálculo.

O valor da SOAM é obtido a partir de um indicador de curvatura $C P$, e é calculado para cada segmento, tal como descrito na Seção 3.6.1.2. Porém, como nosso intuito é obter, também, uma métrica para todos os segmentos que compõem uma rede vascular, usamos como valor SOAM a somatória de todas as curvaturas $C P$ de todos os segmentos, e o dividimos pela soma dos comprimentos de todos os segmentos. Ou seja, para uma rede vascular $(R V)$ formada por $n$ segmentos $\left\{S_{1}, S_{2}, \cdots, S_{n}\right\}$, o valor de SOAM da rede vascular é dado por:

$$
S O A M(R V)=\frac{\sum_{i=1}^{n} C P\left(S_{i}\right)}{\sum_{i=1}^{n} \operatorname{comprimento}\left(S_{i}\right)}
$$

\subsubsection{Ordem de Strahler}

Na Ordem de Strahler, conforme comentado na Seção 3.6.1.3, a atribuição de valores para cada segmento dependo da hierarquia na qual ele se encontra. Estes valores são amplamente usados na análise de grafos, porém, para seu cálculo o grafo tem que ser um grafo enraizado acíclico. Assim, antes de calcular os valores de Strahler faz-se necessário remover os ciclos do grafo e escolher um nó raiz. Esse processo de remoção foi feito por meio do cálculo de uma árvore de espalhamento mínima (Minimum Spanning Tree, MST), que é propagada a partir do primeiro nó criado no grafo de representação. Por exemplo, os valores da Strahler aplicados ao grafo da Figura 4.14 são apresentados na Figura 4.16. 
Figura 4.16: Possíveis valores de Strahler correspondente ao grafo mostrado na Figura 4.14. O vértice vermelho é o ponto inicial e o segmento pontilhado de cor vermelha foi desconsiderado pelo MST.

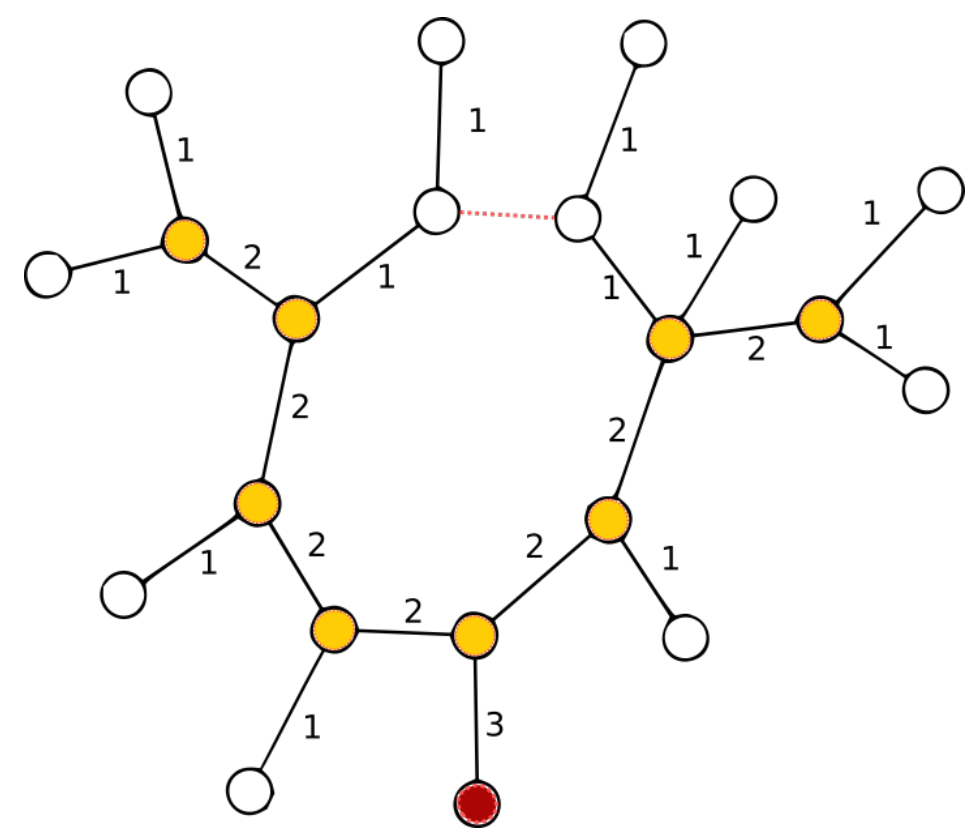

\subsubsection{Taxonomia de Tokunaga}

A taxonomia de Tokunaga é uma extensão da ordem de Strahler. Para sua obtenção usamos a mesma árvore empregada no cálculo dos valores de Strahler, e seus valores são calculados como descrito na literatura (Ver Seção 3.6.1.3). Um exemplo dos valores atribuídos por essa taxonomia é mostrado na Figura 4.17.

A partir dos valores de Tokunaga obtidos anteriormente, geramos a denominada matriz de Tokunaga, a qual consolida todos os valores de Tokunaga e possibilita a análise de estruturas vasculares. Essa matriz é formada por elementos $N_{i j}$, onde o $(i, j)$-ésimo elemento corresponde ao elemento da $i$-ésima linha e $j$-ésima coluna. $\mathrm{O}$ valor de cada elemento $N_{i j}$ é igual à quantidade de segmentos da ordem $i j$ em toda a rede vascular. No entanto, usar unicamente a matriz de Tokunaga pode acarretar alguns problemas na caracterização dos vasos, principalmente por causa do crescimento exponencial dos elementos $N_{11}$ em estruturas mais complexas. Por este motivo, na caracterização da rede vascular consideramos a matriz de proporção [Zoghbi et al., 2014], a qual é definida como $M_{i j}=N_{i j} / N_{j}$, onde $N_{j}$ é a quantidade de segmentos da ordem $j$. A matriz resultante dos valores de Tokunaga, mostrados na Figura 4.17 é apresentada na Figura 4.18. 
Figura 4.17: Valores de Tokunaga correspondente ao grafo mostrado na Figura 4.14

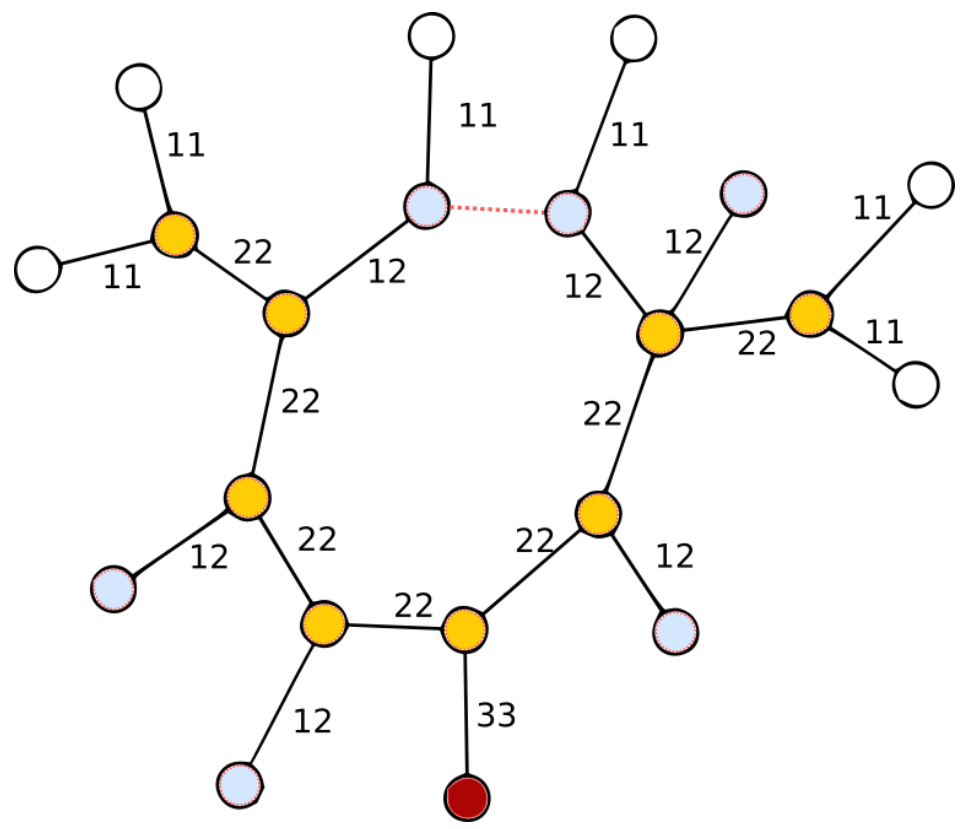

Figura 4.18: Matriz de Tokunaga obtida a partir dos valores da Figura 4.17 e sua representação com intensidades

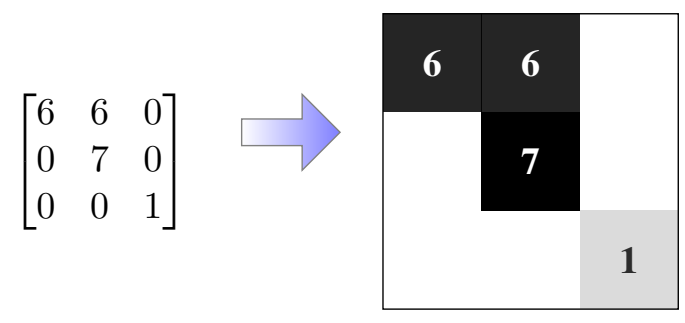

\subsection{Aplicações}

Já que a quantificação de redes vasculares é um processo extremamente útil no processo de diagnostico, as possíveis aplicações da metodologia são inúmeras. Nesta seção descrevemos algumas dessas aplicações.

\subsubsection{Segmentação interativa}

Na literatura existe uma grande diversidade de algoritmos de segmentação, os quais podem ser usados em diferentes condições. Porém, algumas vezes não é necessária a extração de toda a rede vascular de uma imagem, mas apenas de alguns segmentos. Uma abordagem simples para 
esse problema é apagar manualmente os voxels dos segmentos não desejados da rede vascular segmentada. Porém, essa tarefa é um processo repetitivo e entediante, cuja complexidade varia de acordo à ferramenta utilizada.

A partir da nossa modelagem essa tarefa é simplificada. Nossa abordagem possibilita a escolha dos segmentos desejados através dos identificadores a eles atribuídos. Para obter esses identificadores, primeiro executamos todas as instruções da representação obtida e geramos uma imagem, tal como descrito por [Valverde, 2012]; com essa metodologia atribuem-se diferentes intensidades aos voxels. Porém, como desejamos obter os identificadores dos segmentos, atribuímos o mesmo valor de intensidade aos voxels de um mesmo segmento, esse valor é igual ao de seu respectivo identificador. Desta forma, para saber qual é o identificador de um segmento na imagem obtida, bastará escolher um dos seus segmentos, e observar qual a intensidade atribuída a ele. Finalmente, discretizamos somente os segmentos com os identificadores escolhidos.

Dessa forma a complexidade do processo para o usuário é reduzida ao procedimento de escolher apenas um voxel por segmento desejado.

\subsubsection{Correspondência entre redes vasculares}

Esse tipo de problema têm a seguinte forma: considere-se um conjunto de segmentos de vasos sanguíneos em uma imagem inicial; deseja-se obter o conjunto de segmentos que corresponde aos mesmos segmentos numa outra imagem. Abordamos o problema explorando correspondências entre grafos. Nesta abordagem procura-se um emparelhamento entre os segmentos de uma segmentação inicial com poucos segmentos, que denominamos modelo de comparação, e uma outra segmentação (não necessariamente do mesmo individuo) que denominamos de imagem alvo. Para tanto, extraímos o grafo de representação por meio dos procedimentos anteriormente descritos, e comparamos o grafo da segmentação inicial com o grafo obtido da imagem alvo. O método irá comparar todos os segmentos e obter um resultado que maximize a quantidade de acertos.

O problema de correspondência descrito relaciona-se com o problema de extração do $s u b$ grafo comum (estudado na área de teoria de grafos), um problema que se caracteriza pela procura de subgrafos isomorfos entre dois grafos. Trata-se de um processo complexo, tal como é sabido, encontrar um subgrafo isomorfo de um dado grafo é um problema que pertence à classe de problemas NP-completo [Cook e Holder, 2006, p. 20]. Esse problema é o foco de inúmeras pesquisas.

A formalização do problema é descrita a seguir: sejam os grafos não dirigidos $G_{1}=\left(V_{1}, E_{1}\right)$ 
e $G_{2}=\left(V_{2}, E_{2}\right)$. Um grafo $G$ é chamado de subgrafo comum de $G_{1}$ e $G_{2}$, se existe um subgrafo isomorfo de $G$ em $G_{1}$ e de $G$ em $G_{2}$. Um subgrafo comum de $G_{1}$ e $G_{2}$ é chamado de máximo subgrafo comum, se não existe outro subgrafo comum de $G_{1}$ e $G_{2}$ com mais nós que $G$ [Lézoray e Grady, 2012].

Identificar se dois grafos são isomorfos é um processo que requer uma rotulação de vértices e de arestas, de maneira que seja possível determinar um mapeamento entre os vértices e arestas de um grafo em outro grafo. Porém, no caso das redes vasculares, uma artéria pode, por exemplo, apresentar uma bifurcação em um indivíduo e em outro não. Nesse caso duas arestas do grafo teriam que ser relacionadas com apenas uma aresta do outro; e ainda, um vértice poderia existir em um grafo, e no outro, não. Portanto o isomorfismo que devemos procurar é um isomorfismo elástico, que não requer a existência de todos os elementos nos dois grafos. Por este motivo, para esta aplicação, em particular, dedicamo-nos a descobrir o melhor resultado de associação entre os segmentos do grafo. Para tanto, fez-se necessário definir um método de determinar o grau de correspondência entre dois dados segmentos.

O método de detecção de segmentos por nos definido baseia-se no cálculo de distância entre as características de dois segmentos. Para tanto, normalizamos as características obtidas a partir de dois segmentos e os ponderamos com um dado peso. Na sequência calculamos a distância euclideana existente entre esses valores. Ou seja, para dois segmentos $\left(S_{1}\right.$ e $\left.S_{2}\right)$ cujas características são $C_{1}=\left\{\alpha_{1}, \alpha_{2}, \cdots, \alpha_{n}\right\}$, e $C_{2}=\left\{\beta_{1}, \beta_{2}, \cdots, \beta_{n}\right\}$, respectivamente, a distância entre os segmentos, considerando os pesos de cada característica como sendo $W=\left\{w_{1}, w_{2}, \cdots, w_{n}\right\}$, é dada por:

$$
\operatorname{distância}\left(S_{1}, S_{2}\right)=\sqrt{w_{1}\left(\alpha_{1}-\beta_{1}\right)^{2}+w_{2}\left(\alpha_{2}-\beta_{2}\right)^{2}+\cdots+w_{1}\left(\alpha_{1}-\beta_{1}\right)^{2}}
$$

Em nosso caso, escolhemos as seguintes características: comprimento, diâmetro, DM, ICM, SOAM, e a média das distâncias euclideanas entre os pontos iniciais e finais dos segmentos. Estas características foram escolhidas por serem algumas das mais recorrentes nos processos de quantificação de redes vasculares na literatura. Quanto aos pesos, foram escolhidos de forma empírica e seus valores são: 0,$25 ; 0,2 ; 0,15 ; 0,03 ; 0,02$ e 0,35 , respectivamente.

Essa função de distância faz possível quantificar a similaridade entre dois segmentos vasculares, o que é necessário para que se possa encontrar um subconjunto de segmentos da imagem alvo que maximize sua similaridade com os segmentos dados na imagem modelo. Casos como o deste processo constituem um problema fundamental da teoria da análise combinatória. Trata- 
se do chamado problema de atribuição, cuja abordagem mais tradicional é feita por meio do uso do método Húngaro, desenvolvido inicialmente por Kuhn [1955], e que funciona em tempo polinomial.

Para a utilização do método Húngaro [Kuhn, 1955] é necessário calcular a distância de cada um dos segmentos do modelo em relação a todos os segmentos do alvo. A partir dessas distâncias criamos uma matriz bidimensional, e a usamos como entrada para o algoritmo húngaro. Esse algoritmo procura a melhor associação possível entre os segmentos do modelo e do alvo. Como resultado desse processo obtém-se duas listas ordenadas de índices, sendo a primeira delas composta pelos identificadores de segmento da imagem modelo e a segunda, formada pelos identificadores dos segmentos da imagem alvo, de maneira que, o segmento associado ao i-ésimo identificador da primeira lista corresponde ao segmento do i-ésimo identificador da segunda.

Uma vez que, os resultados de correspondência foram obtidos, faz-se possível extrair, a partir da rede vascular alvo, os segmentos relacionados aos segmentos do modelo. Pelo mesmo procedimento é também possível gerar uma nova imagem contendo apenas os segmentos escolhidos. Isto é, os segmentos relativos aos segmentos do modelo. Essa imagem pode ser finalmente considerada como sendo a segmentação do modelo desejados na imagem alvo. 



\section{Capítulo 5}

\section{Resultados}

Neste capítulo são mostrados resultados obtidos nas diversas etapas deste trabalho. Mostramos, por exemplo: os resultados da caracterização automática de redes vasculares sintéticas e reais; a comparação entre as redes vasculares usando as características obtidas; a comparação da arquitetura entre redes vasculares usando a taxonomia de Tokunaga; e finalmente um experimento de correspondência entre redes vasculares, no qual consegue-se detectar de forma automática um dado conjunto de artérias com uma precisão média de $83 \%$. Nesses experimentos, usamos datasets de imagens sintéticas e de imagens reais de angiografia, os quais descrevemos a seguir.

\subsection{Conjunto de imagens}

Para a obtenção dos resultados usamos imagens sintéticas tridimensionais de diferentes arquiteturas e níveis de bifurcação, as quais foram geradas com o método descrito em [Valverde et al., 2013]. Também usamos duas imagens de angiografia reais, nas quais os centros de linha e os diâmetros foram obtidos por procedimentos manuais. Concernente aos testes de correspondência entre redes vasculares, usamos um dataset de 57 imagens, baseadas no projeto Brava [Wright et al., 2013].

\subsubsection{Dataset de imagens sintéticas}

Estas imagens possuem um tamanho padrão de $100 \times 100 \times 100$ e um espaçamento de $1 \mathrm{~mm}$ por voxel. O dataset de teste usado nesta seção foi classificado nos seguintes modelos de vascu- 
latura:

Redes vasculares com segmentos retos: Redes vasculares sintéticas compostas por segmentos que são apenas linhas retas. As imagens de projeção dessas redes vasculares sintéticas são mostradas na Figura 5.1.

Redes vasculares com segmentos regulares com tortuosidade: Redes vasculares sintéticas com padrão de bifurcação regular, na qual todas as bifurcações sempre ramificam-se em dois ramos com a mesma quantidade de segmentos. Nessas vasculaturas, os segmentos sempre apresentam tortuosidade.

Exemplos de imagens como estas são mostradas na Figura 5.2.

Redes vasculares com ramificação não binária: Neste modelo de rede vascular, os dois ramos resultantes de uma bifurcação podem não ter o mesmo número de segmentos. Adicionalmente, os segmentos destas imagens possuem tortuosidade, com exceção do segmento vertical principal.

Exemplos de imagem desse tipo são mostradas na Figura 5.3.

Figura 5.2: Imagens sintéticas de angiografia com padrão de ramificação regular.
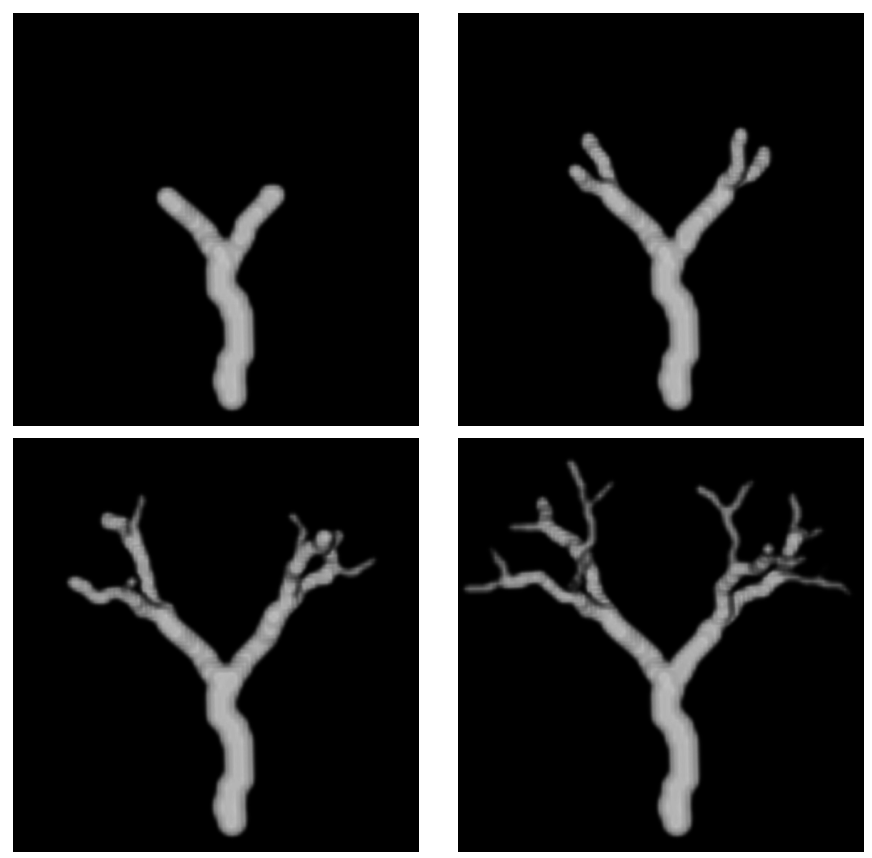
Figura 5.1: Imagens sintéticas de angiografia composta por segmentos retos.
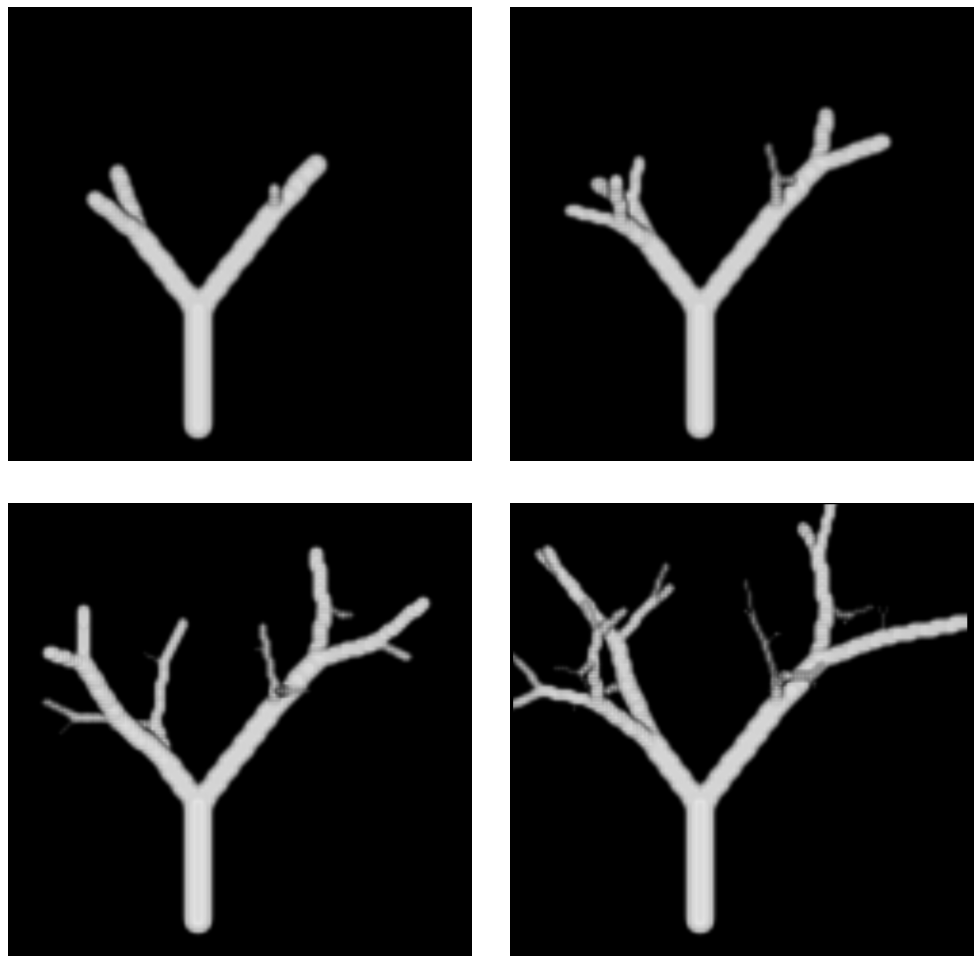

Figura 5.3: Imagens sintéticas de angiografia com padrão e bifurcação não regular.
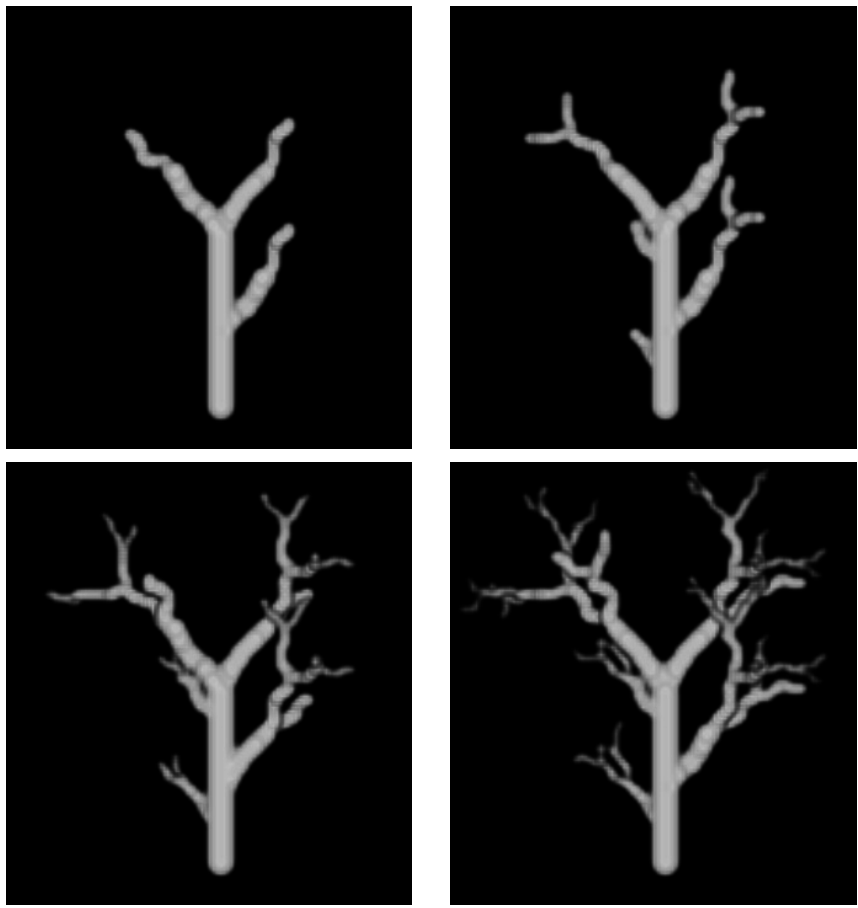


\subsubsection{Dataset de imagens Reais}

Usamos dois datasets de imagens reais, o primeiro deles formado por duas imagens da mesma região, na qual uma delas apresenta um aneurisma, e o outro formado por 57 imagens de artérias cerebrais, as quais são descritas a seguir.

\subsubsection{Imagens de angiografia da artéria carótida interna}

Esse dataset é composto por duas imagens de angiografia real contendo as artérias carótida interna e média direita, as quais foram segmentadas e preprocessadas por procedimentos manuais.

Imagem de controle: A imagem corresponde a um individuo aparentemente saudável.

Paciente com aneurisma: Modelo obtido a partir de uma imagem real de angiografia de um paciente com aneurisma, adquirida da mesma região relativa à da imagem de controle.

As vasculaturas de imagens reais são mostradas na Figura 5.4.

Figura 5.4: Artéria carótida interna e artéria média direita de dois indivíduos, reconstruídas a partir de imagens de angiografia de a) individuo aparentemente saudável b) paciente com aneurisma (indicada pela seta amarela). A região de interesse é mostrada em cor laranja.

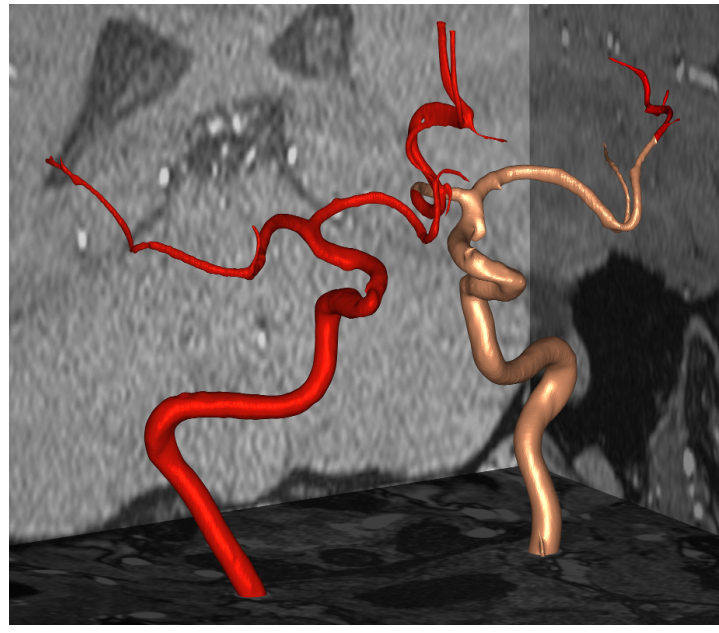

(a)

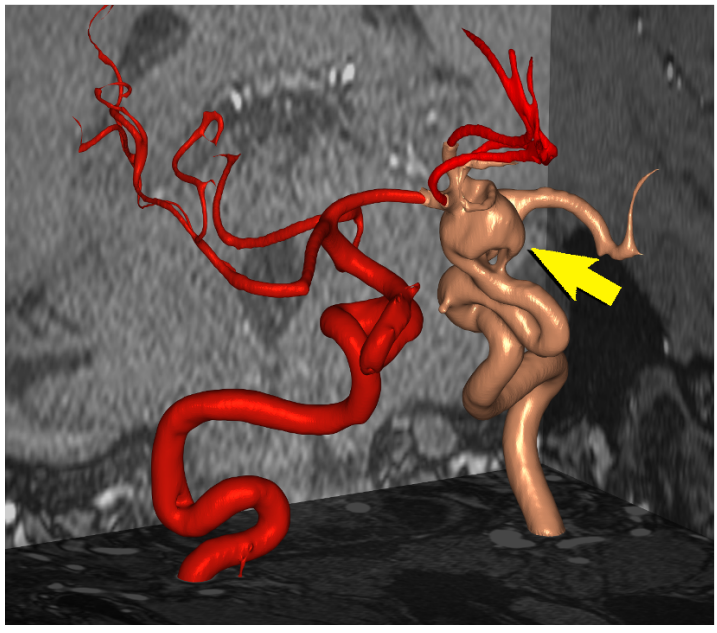

(b) 


\subsubsection{Imagens reconstruídas a partir do projeto Brava}

O projeto Brava ${ }^{1}$ [Wright et al., 2013] disponibiliza dados e imagens de angiografia cerebral de um total de 61 indivíduos aparentemente saudáveis, entre os quais encontram-se 36 mulheres e 25 homens com idade média de 31,2 anos (com um desvio padrão de 10,1). Porém, durante o processamento dos dados, algumas dessas imagens tiveram que ser descartadas, ou pelo fato de se apresentarem arquivos com algum tipo de erro, ou por não ter sido disponibilizada a imagem original do individuo. Dessa forma, foram selecionados os dados de 57 indivíduos.

Figura 5.5: a) Uma imagem real do projeto Brava, b) Projeção da imagem 3D reconstruída das artérias.

(a)

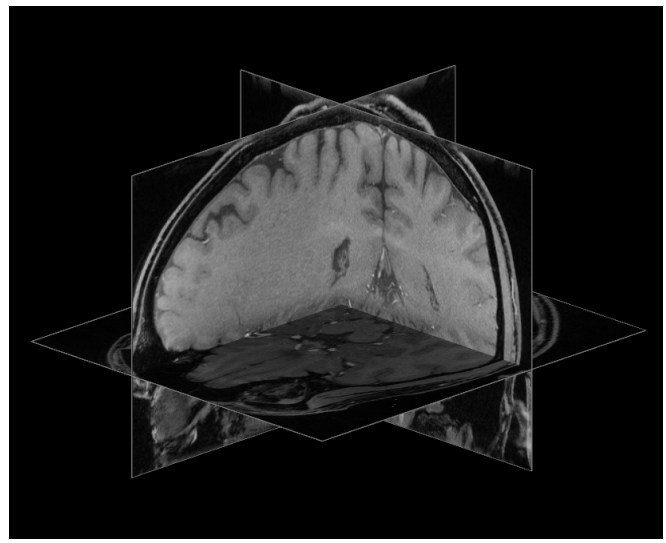

(b)

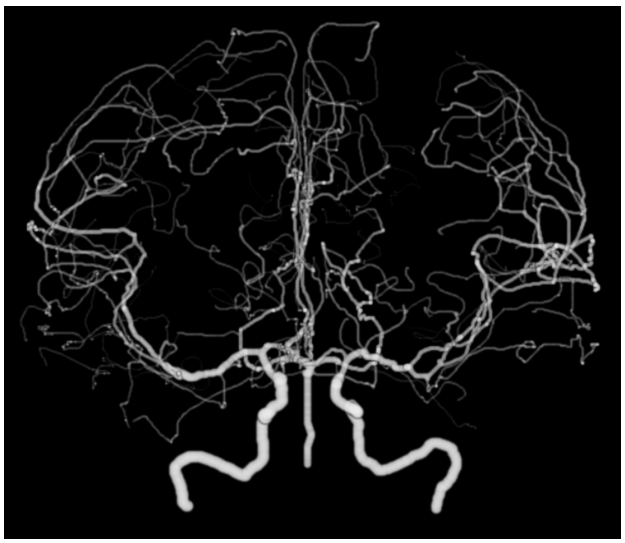

Os dados disponibilizados por esse projeto incluem arquivos de texto, os quais contêm pontos que pertencem ao esqueleto das artérias. Por outro lado, não são disponibilizadas imagens segmentadas, razão pela qual usamos a metodologia de geração de imagens sintéticas de [Valverde, 2012] para a reconstrução de imagens das artérias de cada individuo.

Finalmente, juntamente com os esqueletos publicados pelo projeto Brava, usamos essas imagens como dados de entrada para os experimentos de correspondência. Na Figura 5.5 mostramos uma captura obtida a partir de uma imagem de angiografia de um determinado indivíduo (angiografia publicada pelo projeto Brava), e uma projeção da imagem 3D reconstruída (imagem gerada por nosso procedimento de reconstrução), pertencente ao mesmo individuo. Dita reconstrução foi obtida a partir do esqueleto disponibilizado pelo projeto Brava.

\footnotetext{
${ }^{1}$ http://cng.gmu.edu/brava
} 


\subsection{Avaliação do processo de reconstrução de redes vasculares}

Até este ponto foi descrito o processo de extração de uma representação a partir de uma dada rede vascular, também foram mostrados métodos de extração de características dos vasos diretamente a partir de sua representação. Por outro lado, precisamos validar a hipótese de que, sempre que determinadas características possam ser extraídas de imagens de Angio-RM ou Angio-TC, quer se tratem das características mais usadas na literatura, quer se trate de outras não consideradas no presente trabalho, estas podem também ser extraídas por meio de nossa representação. Isto significaria que nossa representação pode armazenar as informações necessárias para a quantificação da rede vascular.

Figura 5.6: Projeções das imagens $3 D$ reconstruídas com diferentes erros permitidos em vista axial.

(a) Imagem referência

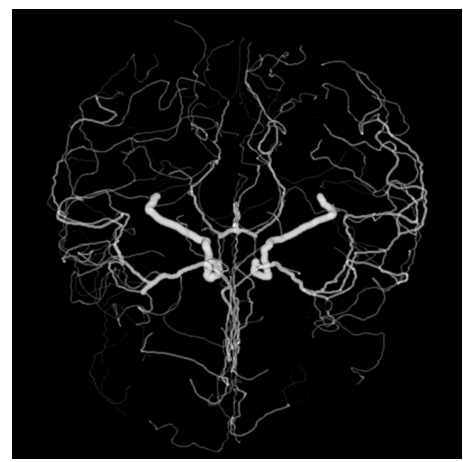

(d) $4 \mathrm{~mm}$ de erro permitido

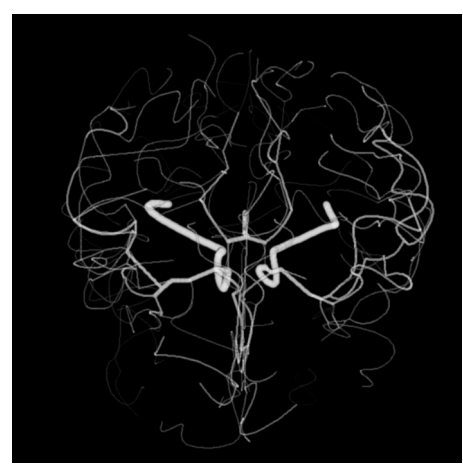

(b) $0,1 \mathrm{~mm}$ de erro permitido

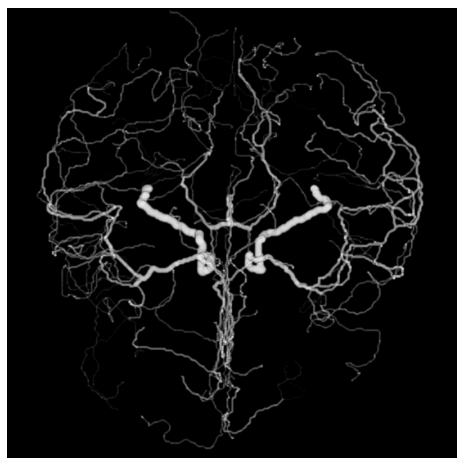

(e) $8 \mathrm{~mm}$ de erro permitido

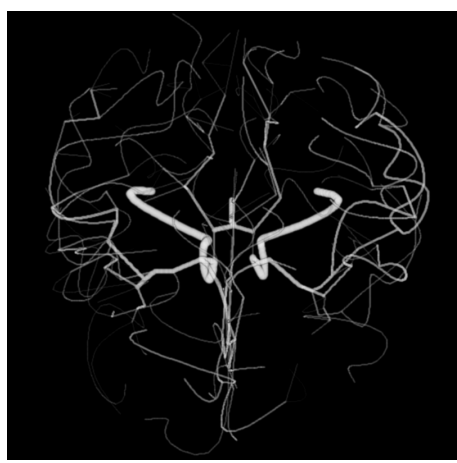

(c) $1 \mathrm{~mm}$ de erro permitido

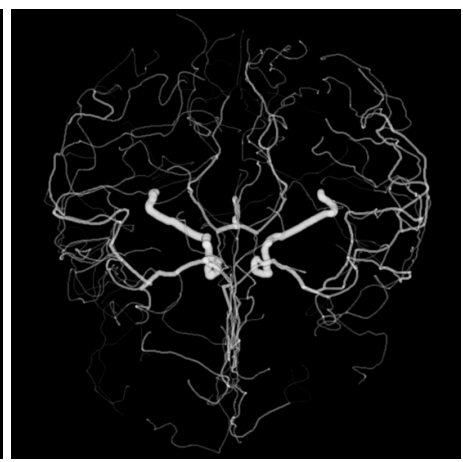

(f) $16 \mathrm{~mm}$ de erro permitido

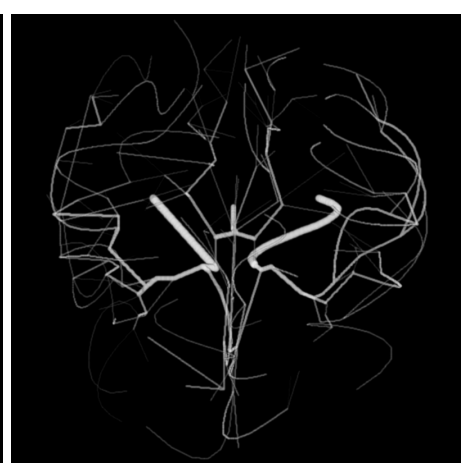

A quantificação pode ser feita por meio da extração das características apresentadas neste trabalho (cujo procedimento foi descrito na seção 4.3), ou pode ser feita também a partir de alguma característica não considerada. Neste caso, se a obtenção de um método de extração 
dessa nova característica não for viabilizada por meio de nossa representação, por meio dela pode ser reconstruída a imagem original; a partir desta imagem pode-se extrair a característica em questão por métodos tradicionais. Podemos afirmar, então, que a representação contêm todas as informações requeridas para quantificar o vaso.

Figura 5.7: Projeções das imagens $3 D$ reconstruídas com diferentes erros permitidos em vista coronal.

(a) Imagem referência

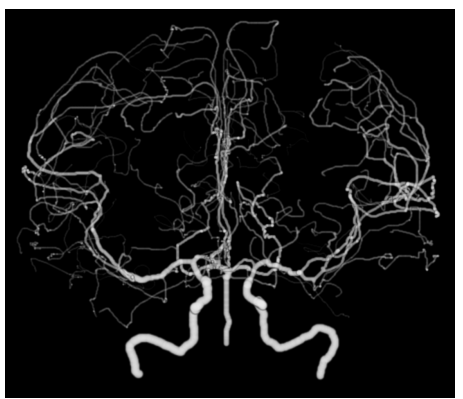

(d) $4 \mathrm{~mm}$ de erro permitido

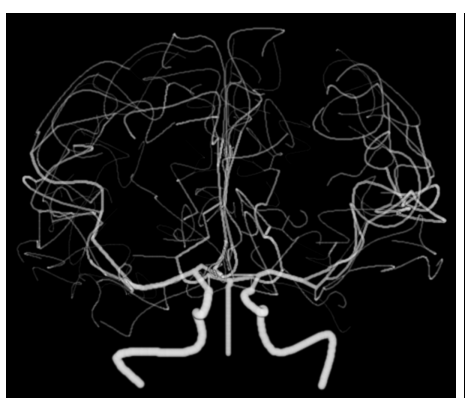

(b) $0,1 \mathrm{~mm}$ de erro permitido

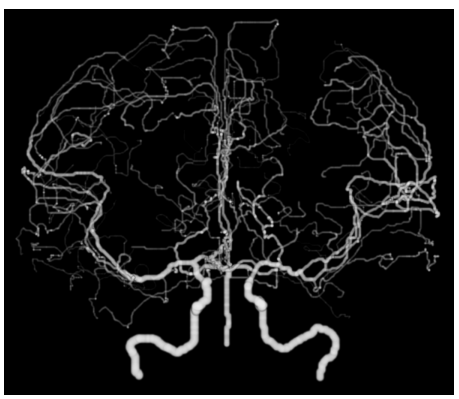

(e) $8 \mathrm{~mm}$ de erro permitido

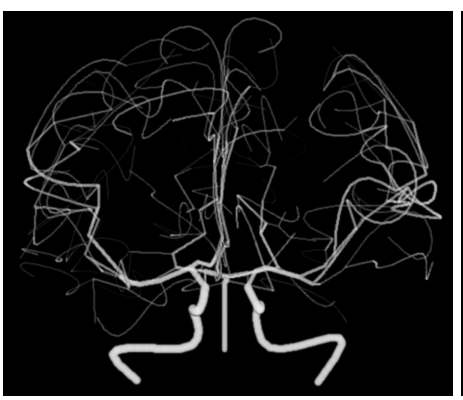

(c) $1 \mathrm{~mm}$ de erro permitido

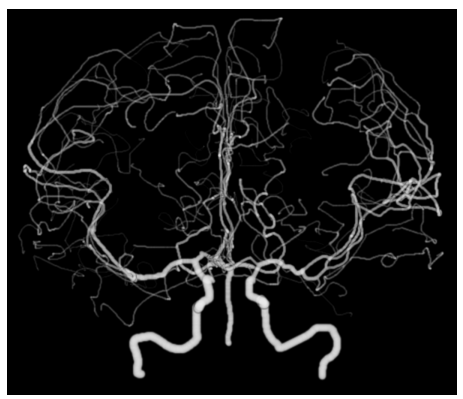

(f) $16 \mathrm{~mm}$ de erro permitido

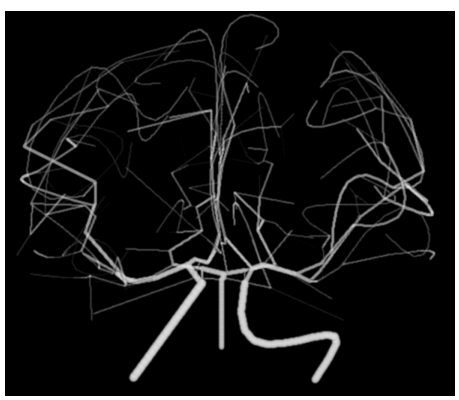

O método de reconstrução tem que gerar imagens que se pareçam o máximo possível com a imagem original. Para avaliar o método usamos o dataset completo de 57 imagens reconstruídas a partir do projeto Brava. Usamos estas imagens como imagens base, e na sequência, extraímos uma nova representação, com diferentes níveis de detalhamento, a partir do esqueleto de cada uma delas. As imagens são comparadas mediante o uso de diferentes medidas. O nível de detalhamento é dado em milímetros pela margem de erro permitido para cada segmento (Ver Seção 4.2.4). A margem de erro é um valor usado no processo de extração de representações a partir das redes vasculares. A partir de uma imagem 3D usada como modelo inicial, bem como de suas reconstruções obtidas com cinco diferentes valores de erro permitido, apresentamos tanto projeções axiais como coronais na Figura 5.7.

Um dos métodos de comparação de imagens voxel a voxel é a raiz do erro quadrático médio 
normalizado (Normalized Root Mean Squared Error, NRMSE), sendo este um dos métodos de comparação de imagens mais conhecidos. Seu valor é dado por:

$$
N R M S E(X, Y)=\frac{\sqrt{\frac{\sum_{i=1}^{n}\left(X_{i}-Y_{i}\right)^{2}}{n}}}{I_{\max }-I_{\min }},
$$

onde $X$ é a sequência de $n$ voxels da imagem considerada como base, $Y$ é a sequência de $n$ voxels da imagem reconstruída de $X ; I_{\max }$ e $I_{\min }$ correspondem às intensidades máximas e mínimas dos voxels, respectivamente. Na Figura 5.7a, mostramos valores da NRMSE para 10 diferentes margens de erro permitidas.

Figura 5.8: Avaliação do processo de reconstrução entre imagens base e suas reconstruções, obtidas em diferentes valores de erro permitido, a) Diferenças com o NRMSE e b) Valores do índice Similaridade estrutural (Structural Similarity, SSIM), no qual 1 é o valor ótimo.

(a)

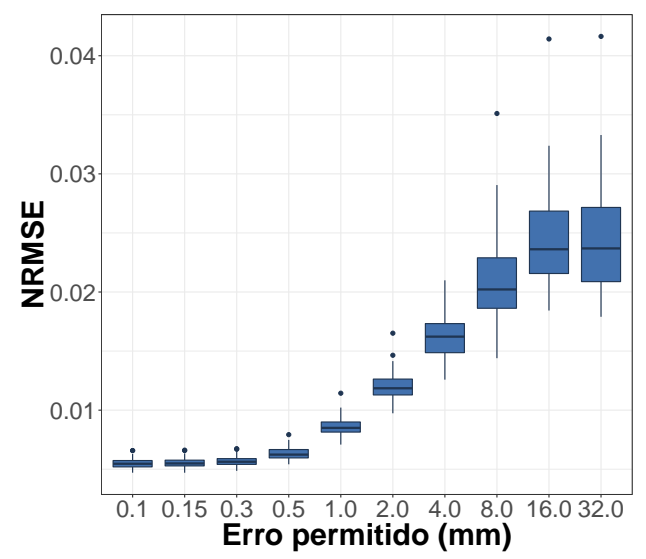

(b)

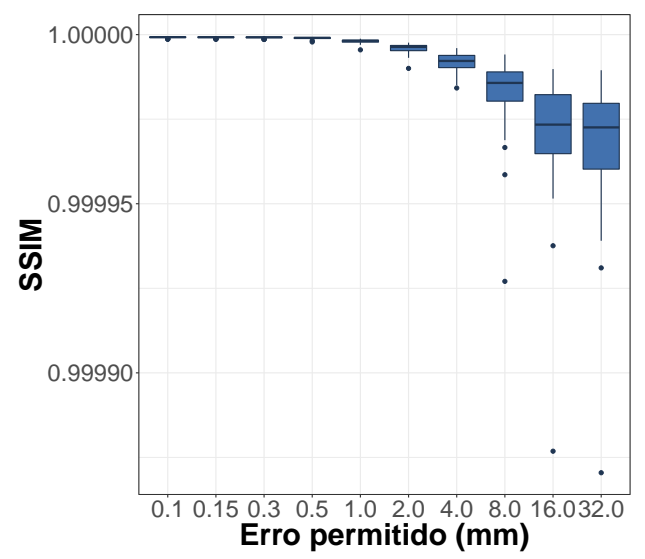

Já na Figura 5.7b, mostramos a comparação das imagens por meio do índice de SSIM [Wang et al., 2004], o qual compara padrões locais de intensidades nos voxels das imagens analisadas, por luminosidade e contraste. Os valores resultantes encontram-se no intervalo entre 0 e 1 , sendo 1 o resultado ótimo. Para o cálculo dessas medidas usamos o dataset completo, e cada imagem do dataset foi comparada com sua respectiva reconstrução, tendo-se em conta diferentes margens de erro.

Adicionalmente, binarizamos tanto as imagens de base como as reconstruídas. Logo, comparamos as imagens voxel a voxel, e contamos a quantidade de verdadeiros positivos, verdadeiros negativos, falsos positivos e falsos negativos. Finalmente, com base nesses valores calculamos a acurácia, a precisão e o Fscore. Os resultados desse processo são mostrados na Figura 5.9. 
Figura 5.9: Comparação entre as binarizações da imagem base e de suas reconstruções com diferentes valores de erro permitido na obtenção da sua representação textual

(a)

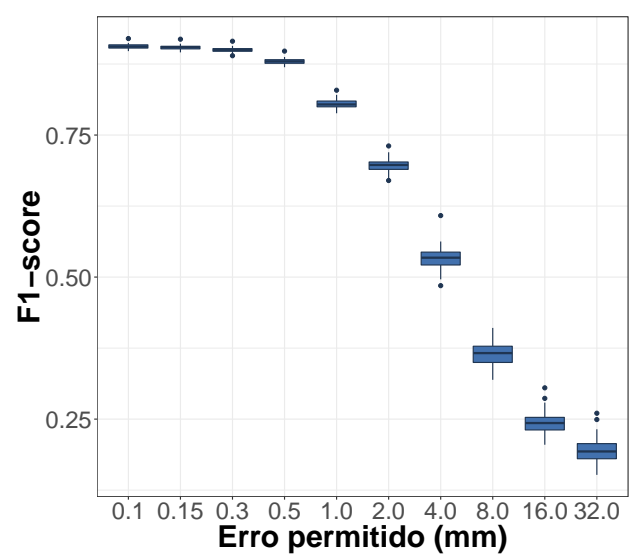

(b)

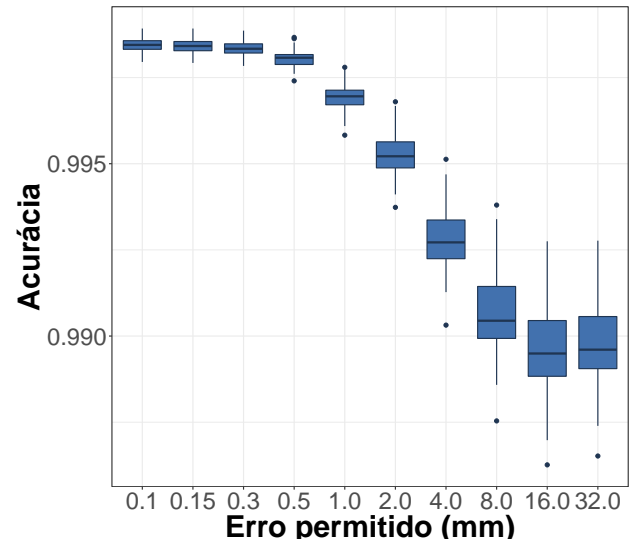

(c)

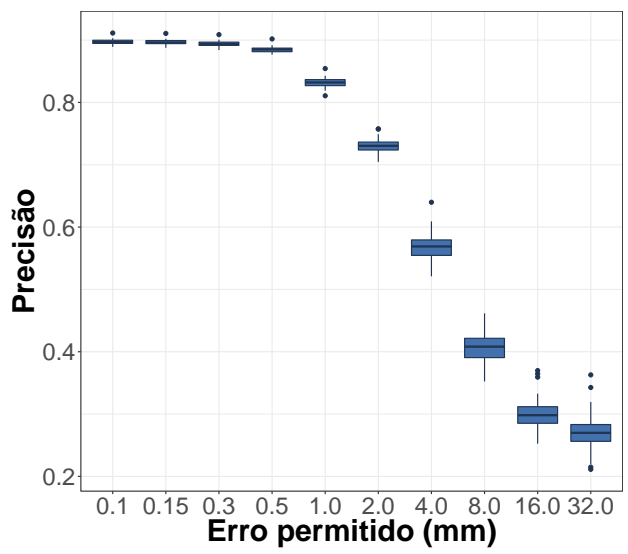

Como podemos observar na Figura 5.8, quanto menor o erro permitido, menor a diferença entre as imagens reconstruídas e a imagem base, obtendo-se menores valores de NRMSE, e valores de SSIM muito próximos ao valor ótimo. Vale destacar que, uma vez que o SSIM analisa padrões locais, e a maioria dos voxels das imagens pertencem ao fundo, os valores apresentados pelo SSIM sempre foram altos. Apesar deste fato, pode-se contudo apreciar a tendência de melhora da imagem que à medida que a margem de erro diminui.

Os resultados de acurácia, precisão e F1-score também mostram a mesma tendência, além disso mostram desvios padrão bastante reduzidos (menor a 0,1 em todos os casos). No entanto, a quantidade de voxels do fundo é extremamente alta, o que faz que os valores dos verdadeiros negativos sejam sempre extremamente elevados. Por esse motivo a acurácia tem resultados mai- 
ores do que 0,95. Quanto aos valores de precisão e F1-score, nos casos em análise, resultaram em valores próximos a 0,5 , tendo-se em consideração uma margem de erro de $4 \mathrm{~mm}$. Portanto, neste caso é melhor levar em consideração medidas que não dependam dos verdadeiros negativos, como é o caso da precisão.

Conforme anteriormente mostrado, quanto maior nível de detalhamento de nossa representação, melhores são os resultados do processo de reconstrução. No entanto, é importante mencionar que o tamanho da nossa representação também aumenta, tal como mostrado na Figura 5.10. Cabe ainda mencionar que mesmo com apenas $0,1 \mathrm{~mm}$ de margem de erro permitido, a quantidade de caracteres obtida foi, em média, menor do que 150000 caracteres. Quanto à maior representação obtida, possuia 171193 caracteres, resultando em um arquivo comprimido (com o algoritmo deflate [Deutsch, 1996] 2) de 37,1 kB. Já o tamanho da imagem base, comprimida com o mesmo método, resultou em $586,6 \mathrm{kB}$, o qual mostra que nosso método pode ser usado também para compressão de imagens.

Figura 5.10: Quantidade de caracteres das representações obtidas com diferentes distâncias de erros permitidas

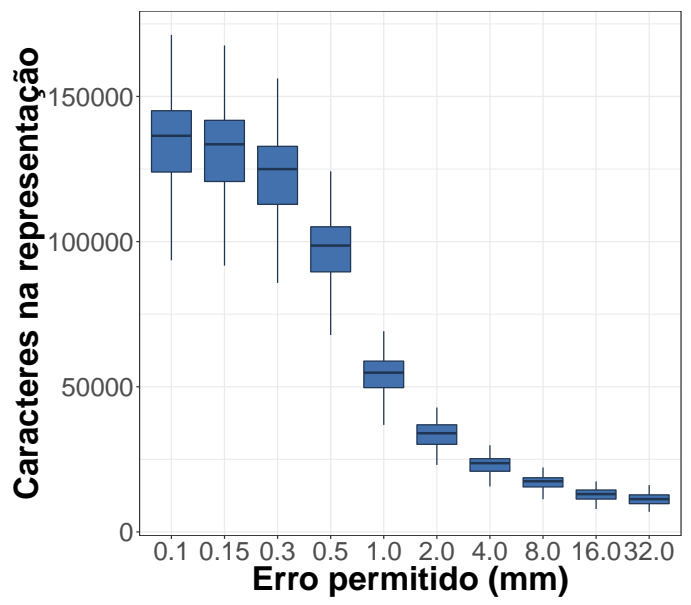

\subsection{Quantificação a partir de imagens sintéticas}

A partir do conjunto de imagens sintéticas de teste, foram calculadas todas as características apresentadas na Seção 4.3. Os resultados são mostrados na Tabela 5.1.

Por meio da observação desses valores é possível perceber que nas estruturas com tortuosi-

${ }^{2}$ o algoritmo deflate é amplamente usado na compressão de arquivos incluindo as imagens NIFTI[Rajna et al., 2015], o arquivo comprimido geralmente tem extensão ".gz" 
Tabela 5.1: Características obtidas a partir do conjunto das imagens de teste.

\begin{tabular}{|c|c|c|c|c|c|c|c|c|c|c|}
\hline $\begin{array}{c}\text { Tipo de } \\
\text { Vasculatura }\end{array}$ & 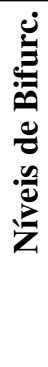 & 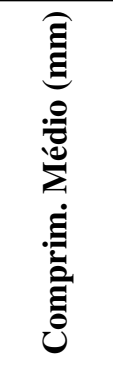 & 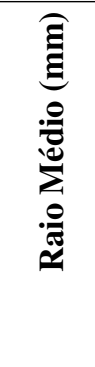 & 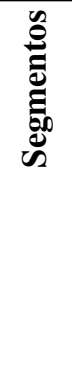 & 気 & 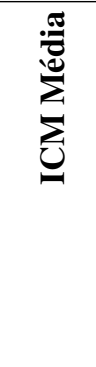 & 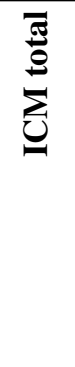 & 主 & 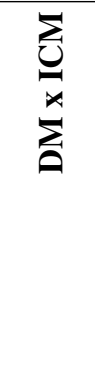 & $\sum_{\infty}$ \\
\hline \multirow[t]{6}{*}{ Com tortuosidade } & 0 & 38.743 & 4.134 & 1 & 0 & 3 & 3 & 1.053 & 3.158 & 0.234 \\
\hline & 1 & 31.046 & 3.491 & 3 & 1 & 3 & 9 & 1.064 & 3.191 & 0.295 \\
\hline & 2 & 26.426 & 2.932 & 7 & 3 & 2.857 & 20 & 1.074 & 3.107 & 0.341 \\
\hline & 3 & 22.724 & 2.411 & 15 & 7 & 2.533 & 38 & 1.084 & 2.814 & 0.389 \\
\hline & 4 & 19.178 & 1.964 & 31 & 15 & 2.548 & 79 & 1.088 & 2.799 & 0.489 \\
\hline & 5 & 16.195 & 1.535 & 63 & 31 & 2.571 & 162 & 1.12 & 2.922 & 0.552 \\
\hline \multirow[t]{5}{*}{ Não binária } & 0 & 48 & 4.09 & 1 & 0 & 1 & 1 & 1 & 1 & 0 \\
\hline & 2 & 39.231 & 3.379 & 5 & 2 & 3.2 & 16 & 1.087 & 4.075 & 0.29 \\
\hline & 5 & 24.053 & 2.76 & 15 & 7 & 2.933 & 44 & 1.084 & 4.157 & 0.357 \\
\hline & 7 & 18.249 & 2.248 & 37 & 18 & 2.568 & 95 & 1.075 & 2.92 & 0.436 \\
\hline & 8 & 17.033 & 1.845 & 71 & 35 & 2.549 & 181 & 1.1 & 2.889 & 0.517 \\
\hline \multirow[t]{5}{*}{ Segmentos retos } & 2 & 23.196 & 2.886 & 7 & 3 & 1 & 7 & 1 & 1 & 0 \\
\hline & 3 & 18.167 & 2.374 & 15 & 7 & 1 & 15 & 1 & 1 & 0 \\
\hline & 4 & 14.094 & 1.849 & 31 & 15 & 1 & 31 & 1 & 1 & 0 \\
\hline & 5 & 11.531 & 1.479 & 60 & 29 & 1 & 60 & 1 & 1 & 0 \\
\hline & 6 & 8.732 & 1.14 & 115 & 57 & 1 & 115 & 1 & 1 & 0 \\
\hline
\end{tabular}


dade a média da ICM diminui à medida que o nível de bifurcação aumenta. Isto ocorre porque muitos segmentos pequenos começam a ser criados, já que segmentos com limitados números de voxels tendem a criar erros na contagem dos pontos de inflexão.

Também é notória a variação das medidas de tortuosidade entre as diferentes estruturas. Por exemplo, no dataset de imagens com segmentos retos, a SOAM é igual a 0, e a DM é igual a 1. Isto se deve ao fato de que seus segmentos não têm tortuosidade, e ao de que o comprimento total de cada segmento é igual à distância entre o primeiro e o último voxel.

Os resultados mostram que a implementação da nossa metodologia, permite extrair as características mais usadas na literatura, as quais podem ser úteis para o diagnóstico automático de determinadas doenças.

Figura 5.11: Imagens sintéticas de angiografia com ramificações regulares, em diferentes níveis de bifurcação, com suas correspondentes matrizes de Tokunaga (Espaços vazios nas matrizes representam o valor 0 ).

(a)
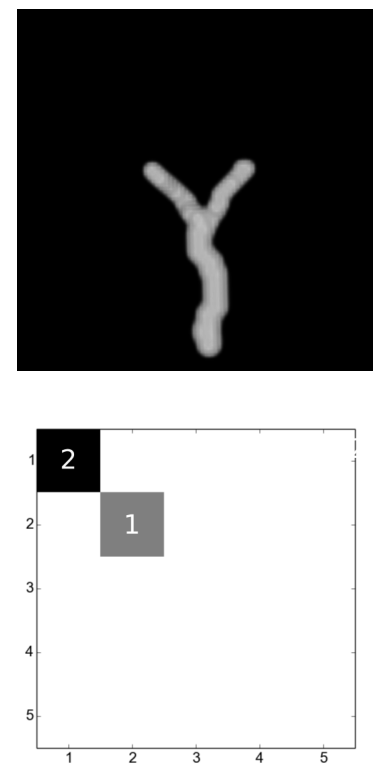

(b)

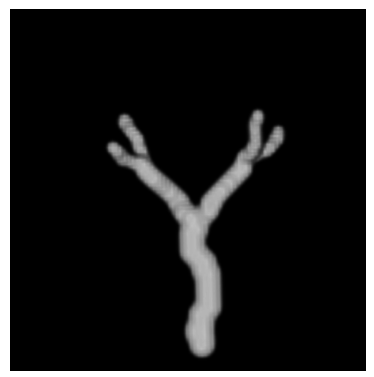

\section{4}

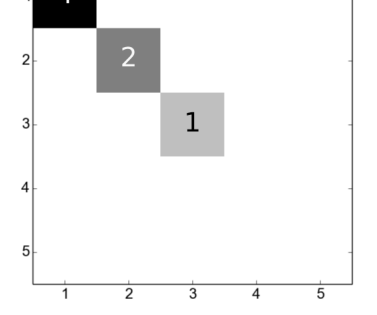

(c)

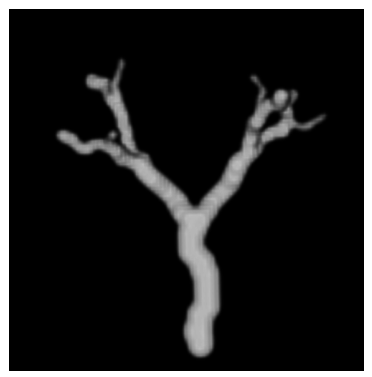

\section{8}

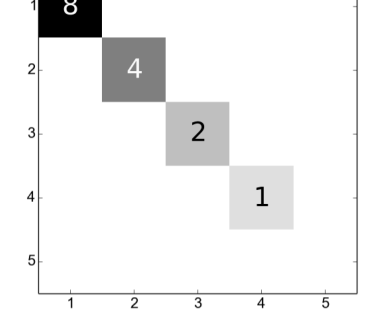

(d)

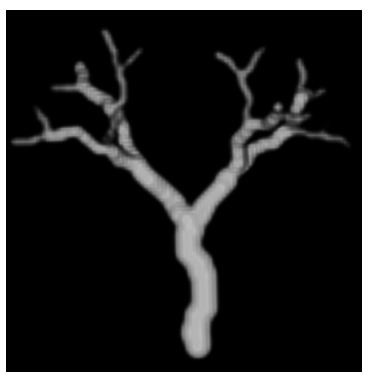

16

4

\subsubsection{Quantificação com a taxonomia de Tokunaga}

Neste experimento, imagens sintéticas de dois tipos de redes vasculares foram usadas: o primeiro deles, mostrado na Figura 5.11, é composto por vasos com bifurcações regulares; o segundo, mostrado na Figura 5.12, é formado por ramificações com padrões irregulares. 
Figura 5.12: Imagens sintéticas de angiografia com padrões de ramificação irregular em diferentes níveis de bifurcação com as matrizes de Tokunaga correspondentes.

(a)

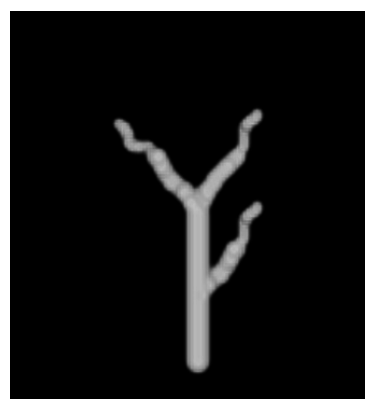

2

2

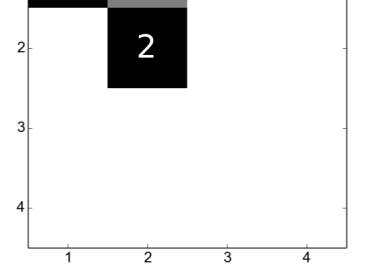

(b)
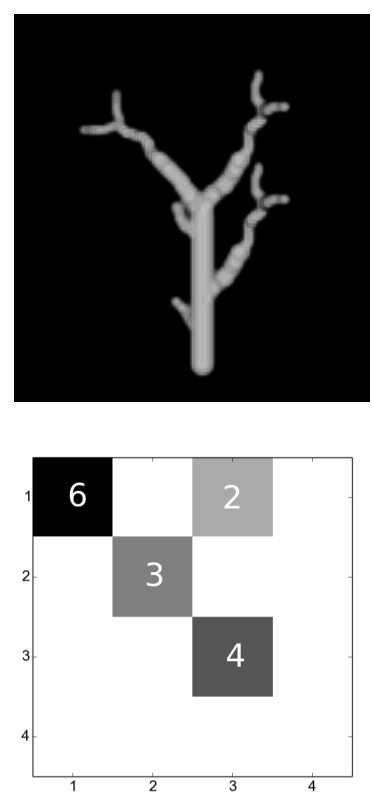

(c)
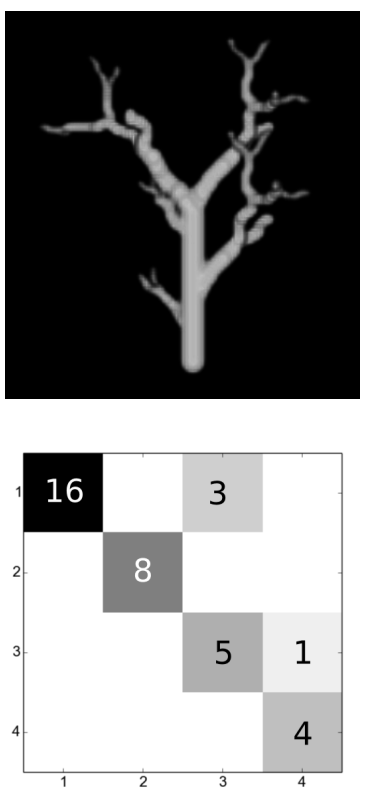

(d)

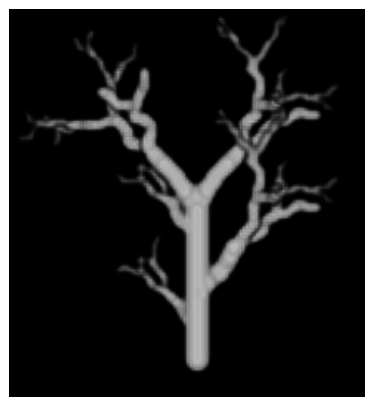

34

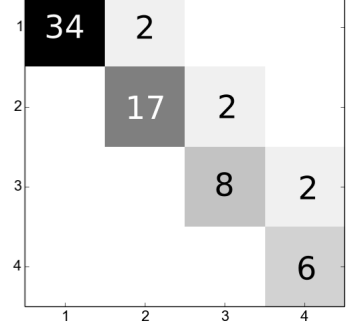

Finalmente, calculamos uma medida de distância definida como a distância Euclideana entre as matrizes de proporção de Tokunaga. Essa distância entre matrizes de Tokunaga permite comparar a complexidade entre arquiteturas vasculares. Na Tabela 5.2 é possível apreciar as distâncias obtidas na comparação das arquiteturas dos vasos sintéticos mostrados na Figura $5.11 \mathrm{e}$ na Figura 5.12. Nessa tabela, podemos ver que a comparação entre estruturas iguais resulta em uma distância nula, e quanto maior a diferença entre as redes vasculares comparadas, maior o valor obtido como distância. Por exemplo, se comparamos uma estrutura simples, formada por apenas uma bifurcação e três segmentos, como a mostrada na Figura 5.11(a), e uma com ramificações bem mais complexas como a da Figura 5.11(d) (que é formada por 31 segmentos com tortuosidade e quatro níveis de bifurcação), obtemos uma distância muito maior em comparação às outras medidas.

\subsubsection{Alinhamento de sequências}

Foi calculada a pontuação dos alinhamentos entre as sequências dos vasos mostrados anteriormente. Os resultados são ilustrados na Tabela 5.3. Eles mostram que o alinhamento entre as 
Tabela 5.2: Comparação entre arquiteturas vasculares mostradas na Figura 5.11 e a Figura 5.12. A cor verde indica mais similaridade e a vermelha maiores diferenças.

\begin{tabular}{|c|c|c|c|c|c|c|c|c|c|}
\hline & \multicolumn{4}{|c|}{ Figura 1} & \multicolumn{4}{|c|}{ Figura2 } \\
\hline & & a) & b) & c) & d) & a) & b) & c) & d) \\
\hline \multirow{4}{*}{$\begin{array}{l}\frac{\sigma}{\pi} \\
\frac{\sqrt{0}}{i}\end{array}$} & a) & 0 & 1 & 1,41 & 1,73 & 0,6 & 1,15 & 1,43 & 1,35 \\
\hline & b) & 1 & 0 & 1 & 1,41 & 1,17 & 0,56 & 1,17 & 1,1 \\
\hline & c) & 1,41 & 1 & 0 & 1 & 1,54 & 1,15 & 0,6 & 0,47 \\
\hline & d) & 1,73 & 1,41 & 1 & 0 & 1,83 & 1,52 & 1,17 & 1,1 \\
\hline \multirow{4}{*}{$\begin{array}{l}\text { 진 } \\
\text { 믄 } \\
\text { 믄 }\end{array}$} & a) & 0,6 & 1,17 & 1,54 & 1,83 & 0 & 1,23 & 1,51 & 1,43 \\
\hline & b) & 1,15 & 0,56 & 1,15 & 1,52 & 1,23 & 0 & 1,05 & 1,23 \\
\hline & c) & 1,43 & 1,17 & 0,6 & 1,17 & 1,51 & 1,05 & 0 & 0,58 \\
\hline & d) & 1,35 & 1,1 & 0,47 & 1,1 & 1,43 & 1,23 & 0,58 & 0 \\
\hline
\end{tabular}

sequências tem uma maior pontuação quando as sequências comparadas são as mesmas do que quando a diferença de complexidade dos vasos é maior, tal como era esperado.

Tabela 5.3: Resultados de alinhamento das sequências dos vasos mostrados na Figura 5.11 e Figura 5.12. As cores são calculadas independentemente para cada fila. A cor verde indica mais similaridade na fila e a vermelha indica maiores diferenças.

\begin{tabular}{|c|c|c|c|c|c|c|c|c|c|}
\hline & \multicolumn{4}{|c|}{ Figura 1} & \multicolumn{4}{|c|}{ Figura 2} \\
\hline & & a) & b) & c) & d) & a) & b) & c) & d) \\
\hline \multirow{4}{*}{$\begin{array}{l}\text { 휸 } \\
\text { 몬 } \\
\text { in }\end{array}$} & a) & 82 & 70,3 & 60 & 35,7 & 54 & 62,2 & 60,9 & 29,1 \\
\hline & b) & 70,3 & 224 & 192,1 & 178,4 & 85,7 & 128,9 & 178,8 & 170,8 \\
\hline & c) & 60 & 192,1 & 482 & 398,7 & 86,6 & 148,8 & 324,7 & 383,3 \\
\hline & d) & 35,7 & 178,4 & 398,7 & 976 & 62,3 & 133,3 & 363,6 & 669,8 \\
\hline \multirow{4}{*}{$\begin{array}{l}\text { N } \\
\text { 윤 } \\
\text { 荥 }\end{array}$} & a) & 54 & 85,7 & 86,6 & 62,3 & 110 & 97,6 & 89,5 & 58,5 \\
\hline & b) & 62,2 & 128,9 & 148,8 & 133,3 & 97,6 & 182 & 161,7 & 130,3 \\
\hline & c) & 60,9 & 178,8 & 324,7 & 363,6 & 89,5 & 161,7 & 448 & 379,6 \\
\hline & d) & 29,1 & 170,8 & 383,3 & 669,8 & 58,5 & 130,3 & 379,6 & 1068 \\
\hline
\end{tabular}

\subsection{Quantificação a partir de imagens reais}

Testes foram feitos usando imagens reais de angiografia adquiridas da mesma região anatômica de dois indivíduos: um indivíduo de controle e o outro com um aneurisma. As características dessas duas redes vasculares foram quantificadas e os resultados são mostrados na Tabela 5.4. 
Tabela 5.4: Características obtidas a partir do conjunto de imagens de exemplo.

\begin{tabular}{|c|c|c|c|c|c|c|c|c|c|c|}
\hline $\begin{array}{c}\text { Tipo de } \\
\text { Vasculatura }\end{array}$ & 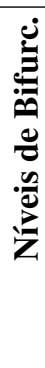 & 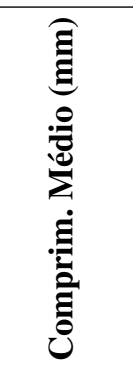 & 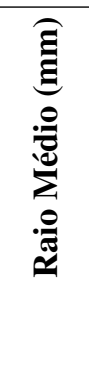 & 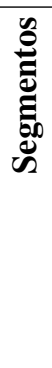 & 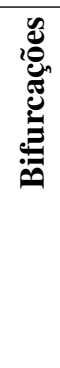 & 苞 & 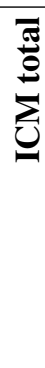 & $\sum$ & 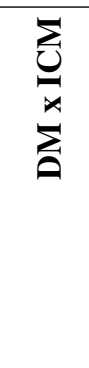 & 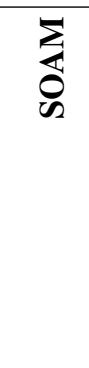 \\
\hline De controle & 2 & 117.088 & 2.703 & 5 & 2 & 2.8 & 14 & 1.716 & 7.994 & 0.067 \\
\hline Com Aneurisma & 3 & 112.067 & 2.432 & 7 & 3 & 2.714 & 19 & 1.808 & 8.374 & 0.075 \\
\hline
\end{tabular}

Também foi feito o cálculo de distância com a taxonomia de Tokunaga nas duas imagens de angiografia de artérias cerebrais reais, sendo que as linhas centrais foram manualmente calculadas. A distância entre as duas imagens, mostradas na Figura 5.4, foi de 0,99, o que reflete diferenças na estrutura. No entanto, é necessário o uso de mais imagens do mesmo tipo para conseguir uma distância que discrimine um vaso saudável de um vaso doente.

\subsection{Correspondência entre redes vasculares}

O objetivo deste experimento é mostrar o desempenho do método de correspondência proposto. Para tanto, necessitamos de um modelo base e de um data-set de imagens segmentadas. Tendo o modelo como base de comparação, procuramos, em cada imagem do dataset, os segmentos que correspondam ao modelo. Neste caso, usamos o dataset liberado pelo projeto Brava, e escolhemos aleatoriamente uma das suas imagens; a partir dela segmentamos as principais artérias que pertencem ao CoW, e usamos a segmentação como modelo. Na sequência extraímos as correspondências do modelo de cada uma das outras imagens, usando o método mostrado na Seção 4.4.2.

O Modelo base usado é mostrado na Figura 5.13, nela, podem ser observados os vasos do CoW segmentados e etiquetados. Na Figura 5.13(b) mostramos sua posição em relação às demais artérias. A orientação dessas e das próximas imagens é mostrada na Figura 5.14. É importante destacar que os esqueletos disponibilizados pelo projeto Brava não incluem uma pequena artéria chamada Artéria Comunicante anterior, a qual fecha o ciclo no CoW. Por tal motivo, esta artéria não é incluída no modelo. 
Figura 5.13: Segmentação do CoW, usada como base de comparação (identificador de imagem BG0003) a) modelo mostrando os nome dos vasos principais do CoW b) artérias no cérebro com destaque às artérias do CoW.

(a)
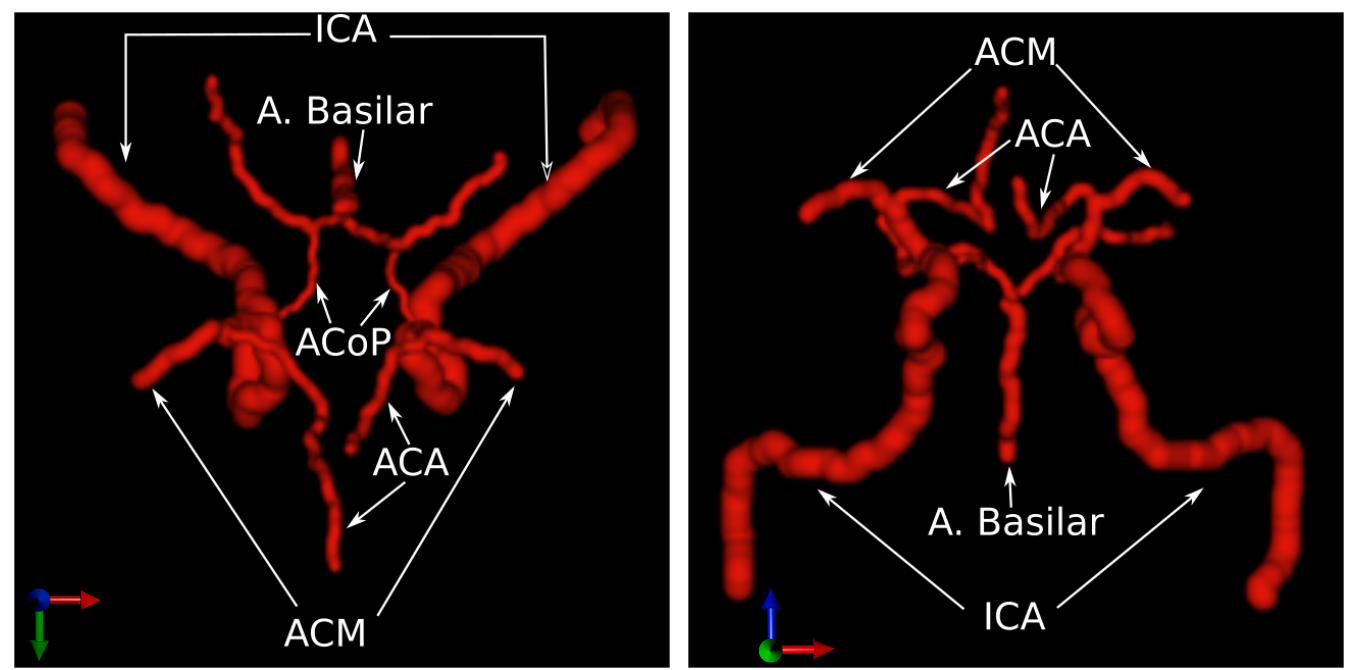

(b)
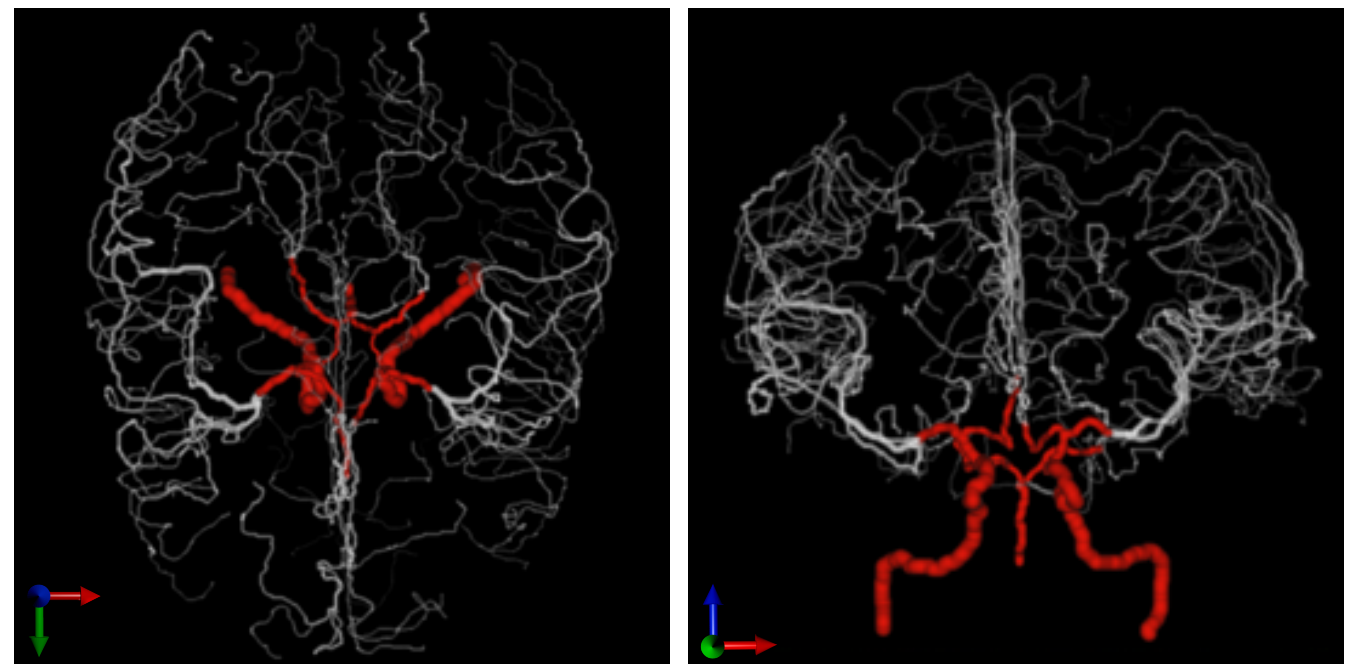

No experimento usamos as 57 redes vasculares consideradas anteriormente. Nestas redes procuramos os segmentos que correspondem aos segmentos do modelo base. Como resultado, em 6 delas foram encontrados todos os segmentos analisados, sendo que consideramos que um segmento foi detectado se pelo menos um fragmento do segmento foi identificado. Um bom resultado de correspondência é, por exemplo, mostrado na Figura 5.15. Nela é possível apreciar 
Figura 5.14: Eixos de orientação

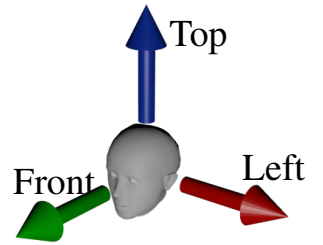

que todos os segmentos existentes na base de comparação foram corretamente detectados.

Tabela 5.5: Precisão das diferentes artérias do CoW.

\begin{tabular}{l|c}
\hline Artéria & Precisão \\
\hline ACI direita & 0,9474 \\
ACI esquerda & 0,9825 \\
ACA direita & 0,6316 \\
ACA esquerda & 0,9474 \\
Artéria basilar & 0,9298 \\
ACoP direita & 0,8596 \\
ACoP esquerda & 0,7544 \\
Artéria cerebral média (ACM) direita & 0,8421 \\
ACM esquerda & 0,8421 \\
\hline Média & 0,8324 \\
\hline
\end{tabular}

Os segmentos que foram detectados nos resultados de correspondência, foram também usados no cálculo da precisão, que mostra a quantidade de vezes em que pelo menos um fragmento do segmento é adequadamente escolhido no processo de correspondência. Esses valores são sumarizados na Tabela 5.5, nessa tabela mostramos a precisão obtida para os segmentos que conformam o CoW. A precisão é obtida da seguinte forma:

$$
\text { Precisão }=\frac{T P}{T P+F P}
$$

onde, TP é a quantidade de vezes em que pelo menos um fragmento do segmento de uma dada artéria foi corretamente escolhida no processo de emparelhamento, e FP é a quantidade de vezes em que a mesma artéria não foi selecionada no processo. Dessa forma, obtivemos a precisão das principais artérias que constituem o CoW.

Nossos resultados mostraram uma precisão média de $83,24 \%$ em todas as artérias, o que é 
Figura 5.15: Resultados de correspondência em uma imagem, na qual todos os segmentos foram detectados (identificador de imagem: $\mathrm{BH} 0018$ )

(a)
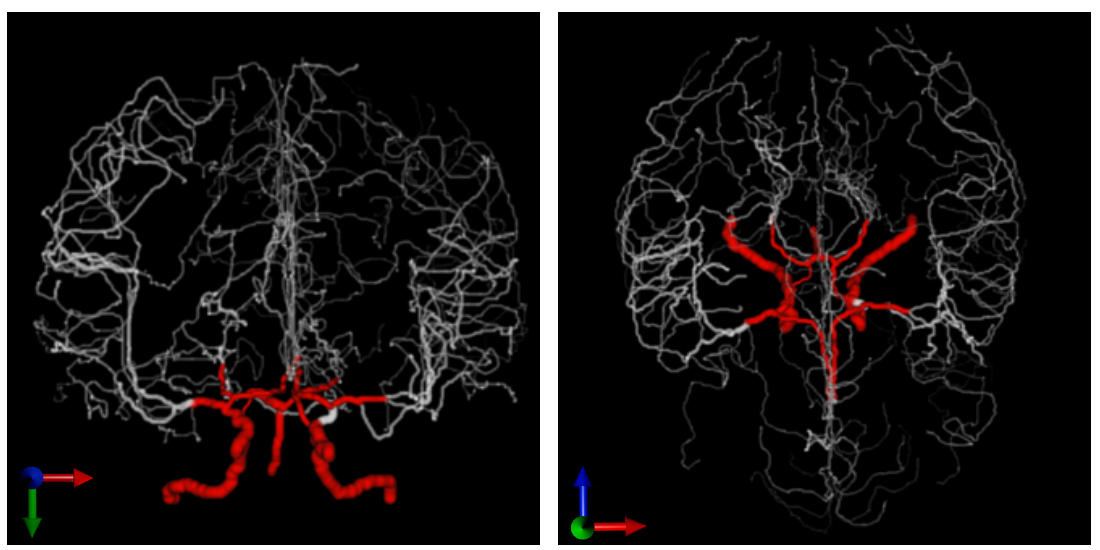

(b)
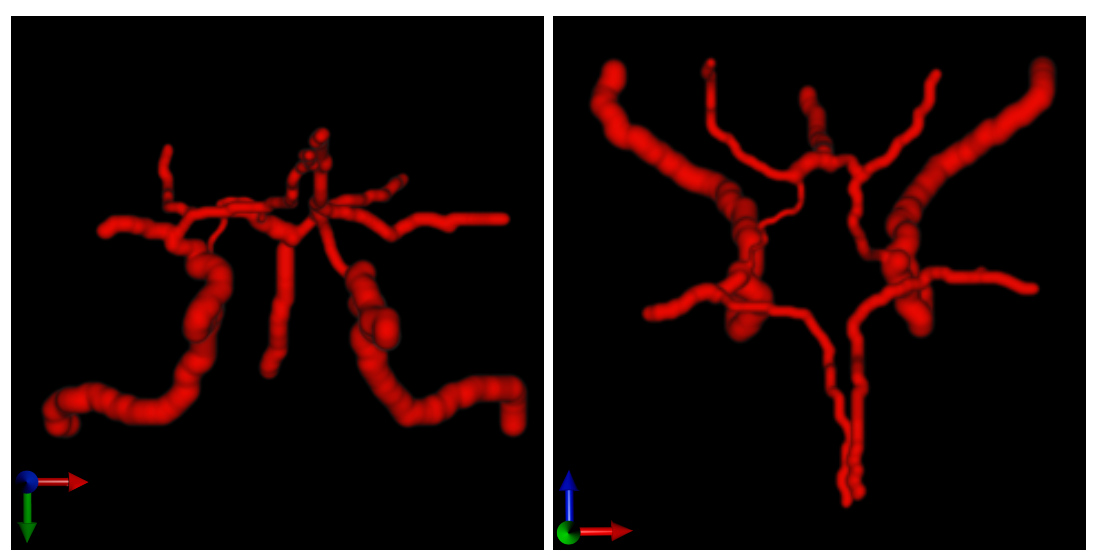

um resultado bom considerando-se a grande quantidade de segmentos existentes nas imagens usadas. Pudemos observar que as artérias carótidas internas tiveram uma alta taxa de acerto com uma precisão média de $96,5 \%$. O resultado se deve sobretudo à grande diferença que existe entre esse vaso e os outros vasos do modelo, tanto em comprimento como em calibre. Um exemplo de identificação correta dessa artéria é mostrado na Figura 5.16.

Também a posição anatômica em que essas artérias se encontram incrementa as chances de ser corretamente classificado. Tanto é assim que nos casos nos quais a artéria foi detectado erroneamente, o erro ocorreu em imagens cujo modelo encontrava-se deslocado em relação à posição do modelo base. Este problema pode ter sido originado no processo de adquisição das imagens. 
Figura 5.16: Segmentos da ACI corretamente detectado em uma imagem vista desde duas orientações.
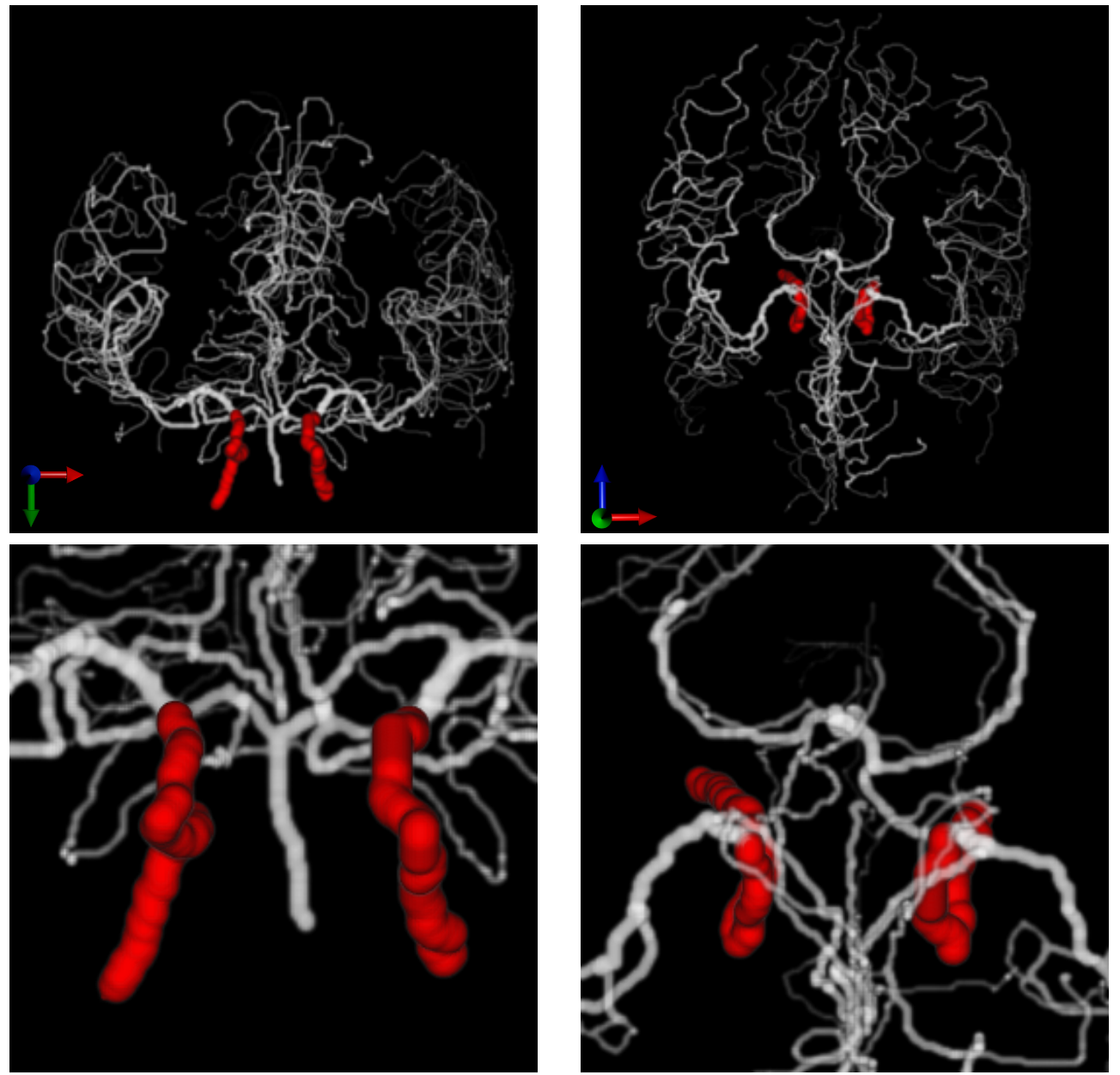

Outra das artérias que apresentou boa precisão (92,98\%) na análise de correspondências foi a artéria Basilar, mostrada na Figura 5.19. Essa artéria, assim como as ACIs, têm características diferentes das outras artérias, em especial a direção, a tortuosidade e a localização da artéria. As artérias ACoP, mostradas na Figura 5.18, tiveram resultados dentro da média, já que o calibre do vaso pode ser confundido com segmentos vizinhos.

Quanto à artéria com menor taxa de acerto, foi a ACA direita. Um fator que pode ter sido decisivo nesse resultado é o tamanho desta artéria no modelo dado como base de comparação. Uma melhor escolha do modelo base pode gerar melhores resultados. 
Figura 5.17: Segmentos da ACA corretamente detectado em uma imagem vista desde duas orientações.
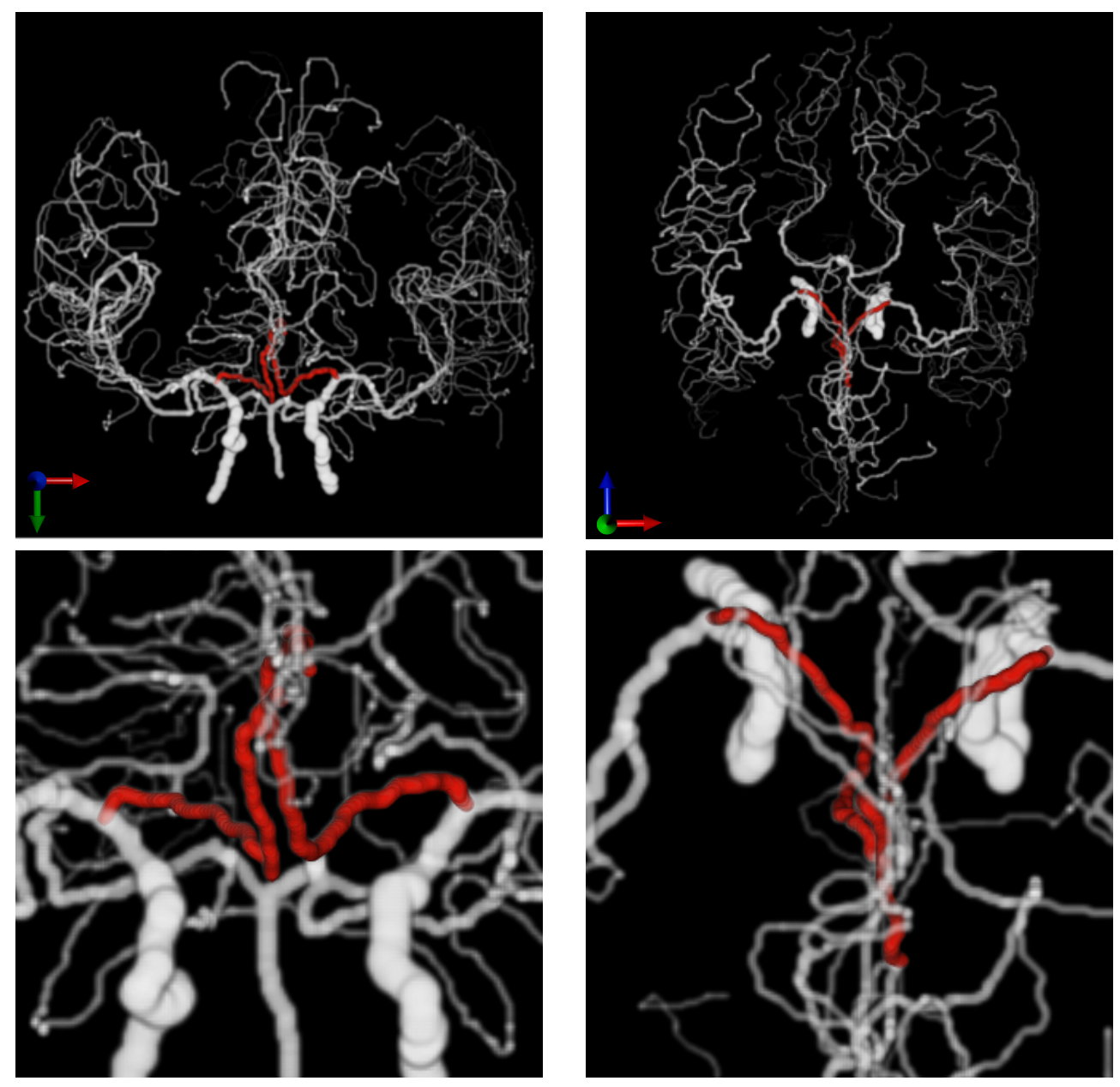
Figura 5.18: Segmentos da ACoP corretamente detectado em uma imagem vista desde duas orientações.
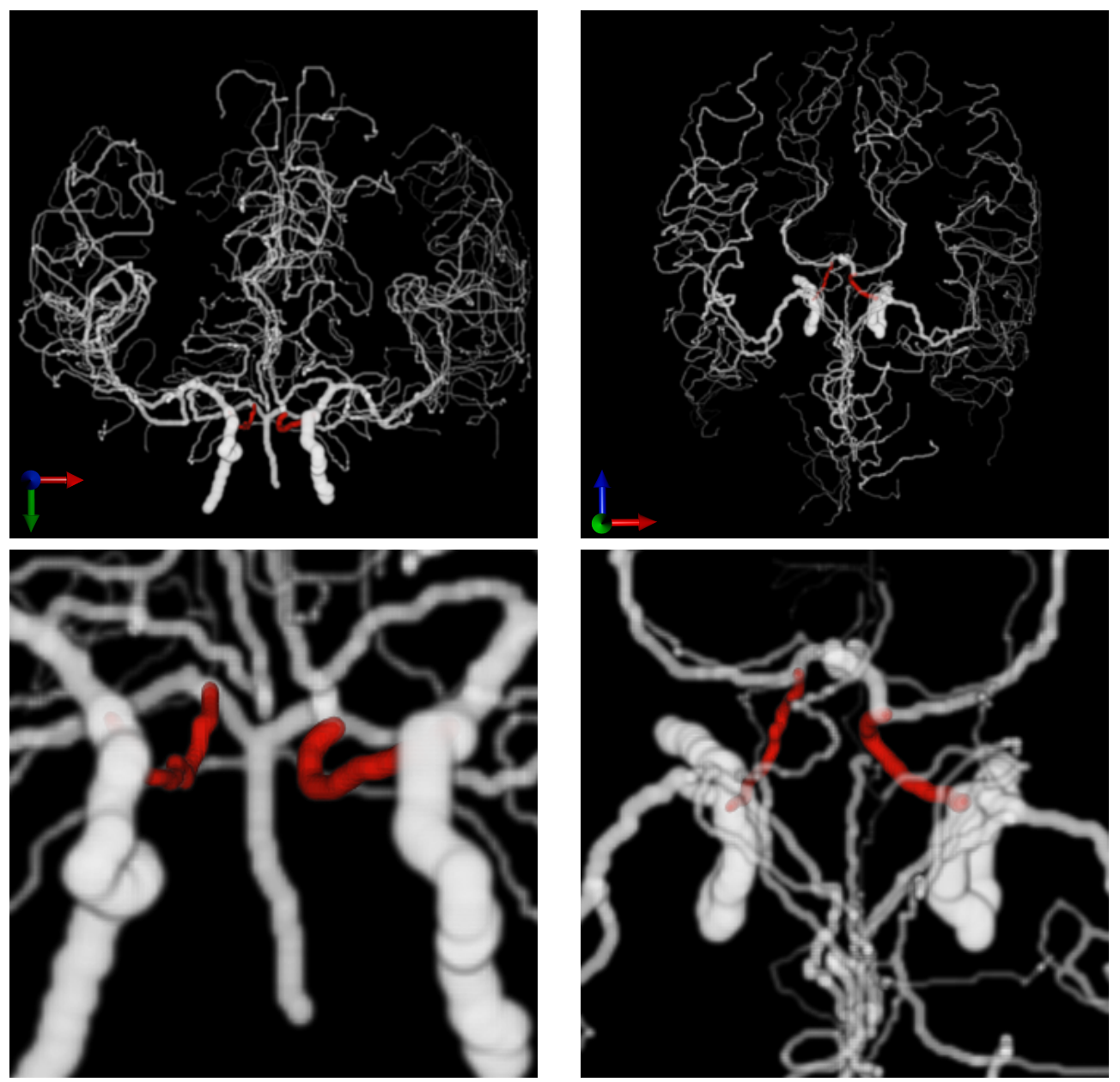
Figura 5.19: Segmentos da artéria Basilar corretamente detectado em uma imagem vista desde duas orientações.
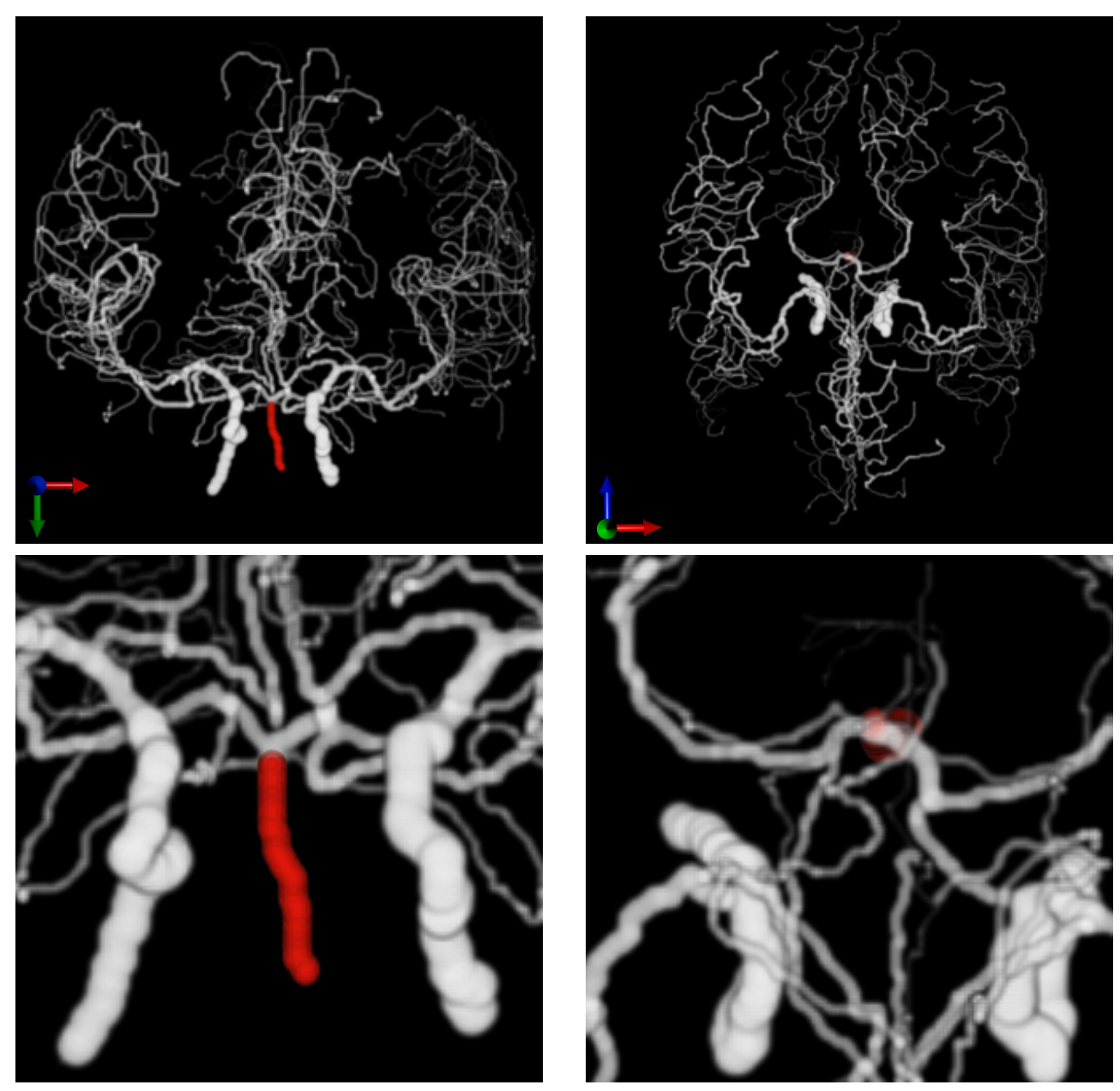


\section{Capítulo 6}

\section{Conclusões}

\subsection{Síntese do trabalho}

A análise de imagens de Angio-TC e Angio-RM são etapas fundamentais no processo de diagnóstico de doenças cardiovasculares, responsáveis por aproximadamente $20 \%$ de todas as mortes em pessoas maiores de 30 anos no 2009, e a principal causal de morte no 2012. A automação do processo de diagnóstico depende em grande medida do processo de quantificação das redes vasculares. Este processo é feito usualmente a partir de imagens.

Neste trabalho apresentamos uma metodologia para a modelagem de redes vasculares e sua quantificação, conforme descrito na Capítulo 4. No processo de modelagem é gerada uma sequencia de instruções que são representadas em forma de cadeias de texto, tal como foi mostrado no Seção 4.2. Essa representação pode ser obtida a partir de imagens de Angio-TC ou AngioRM. Fazendo-se uso dessa representação é possível extrair diversas características morfológicas e topológicas dos vasos, tais como, por exemplo: os raios, os comprimentos, as coordenadas de pontos de bifurcação, dentre outras características discutidas na Seção 4.3. Mostramos também que é possível reconstruir as imagens, o que possibilita a criação de imagens tridimensionais similares às originais. Isso, faz com que a representação, além de diminuir a complexidade computacional do processo de quantificação, possa ser usada para a compressão de datasets. Na sequência, na Seção 4.4, foram mostradas algumas aplicações, entre as quais encontram-se a detecção automática de doenças, a correspondência e rotulação de vasos, e a segmentação interativa.

Para a obtenção dos resultados foi necessário implementar a metodologia completa. Para tanto, foram escolhidas as linguagens Python e C++. A implementação da nossa metodologia 
fez possível a automação do processo completo, que inclui: a esqueletização, a obtenção das linhas centrais dos vasos, a detecção dos pontos destacados dos segmentos, a quantificação, e até mesmo a reconstrução.

Os resultados apresentados na Seção 5.2 mostram que é possível reconstruir as imagens de angiografia 3D originais a partir de nossa representação, o que significa que qualquer característica que possa ser obtida da imagem original, pode ser também obtida fazendo uso da nossa representação. Além disso, desenvolvemos e descrevemos técnicas de quantificação a partir da representação textual descrita, técnicas que permitem extrair características frequentemente usadas na literatura sem ter-se a necessidade de reconstruir a imagem original. Esses resultados são mostrados na Seção 5.3.1. Outras características, como as matrizes de Tokunaga e o índice de Strahler, comumente usadas para a análise de bacias hidrográficas, foram usadas na Seção 5.3.1, obtendo-se bons resultados na comparação de arquiteturas vasculares.

Também mostramos que é possível o uso de algoritmos de sequenciamento genético tendo como base a nossa representação, já que ela é baseada em cadeias de texto. Os resultados do experimento, que usa o algoritmo de alinhamento de sequências para quantificar a similaridade entre vasos, são mostrados na Seção 5.3.2.

Finalmente, na Seção 5.5 mostramos resultados da aplicação da metodologia para a comparação de segmentos de diferentes redes vasculares. Nesse experimento mostramos que é possível detectar determinados segmentos dentro de uma rede vascular complexa. Nesse caso, a precisão média obtida foi de $83,24 \%$. Esse experimento pode ser a base para novas aplicações, tais como a rotulação automática de vasos, a segmentação de determinados vasos, a analise de segmentos selecionados, a busca de segmentos com uma determinada doença (como aneurismas e estenose), entre outras.

\subsection{Contribuições}

O trabalho ora apresentado, no momento, traz três principais contribuições: uma modelagem híbrida dos vasos, que faz uso tanto de cadeia de texto como de grafos, e que permite não apenas a quantificação dos vasos, mas também sua reconstrução; a metodologia de extração automática dessa modelagem a partir de imagens de redes vasculares tridimensionais, e foram introduzidos também extratores, que permitem a obtenção de diversas características a partir da representação, o qual o consideramos como um avanço no caminho ao diagnóstico automático. Além disso, consideramos que o presente trabalho pode ser um ponto inicial para inúmeras pesquisas na área de análise e quantificação de vasos, e no diagnóstico automático de doenças cardiovasculares. 


\subsection{Limitações}

Dados os resultados alcançados, é possível quantificar os vasos por meio de diversas características obtidas a partir da nossa representação. Porém, a escolha das características a serem usadas depende da aplicação, e portanto, pesquisas aprofundadas nesse escopo deverão ser feitos.

A pesquisa limitou-se à análise de redes vasculares tridimensionais obtidas por Angio-RM e Angio-TC, porém, a metodologia pode ser aplicada em imagens adquiridas por outras técnicas, desde que seja providenciado um adequado resultado de segmentação. Além disso, o estudo pode ser generalizado também para abarcar resultados de imagens em duas dimensões. No entanto, nesse caso, deve-se ter cuidado adicional na hora de processar bifurcações, já que em imagens dessa natureza podem existir superposições de vasos.

Nos experimentos de correspondência entre vasos foi atingida uma alta precisão. Porém, devido à natural alteração na posição das artérias das diversas imagens utilizadas, e às diferenças biológicas que existem entre os mesmos vasos de diferentes indivíduos, algumas imagens (duas das 57) tiveram uma taxa de erro elevada (7 e 9 segmentos não detectados, de um total de 15) em comparação com as outras, sendo que a média de segmentos não detectados foi de 2,2 . Isto sugere que melhores resultados podem ser obtidos com uma etapa de registro inicial.

\subsection{Trabalhos futuros}

Consideramos que a metodologia apresentada pode ser o início de muitas pesquisas futuras, tanto na área de ciências da computação, quanto na da biologia e na da medicina. As possíveis aplicações vão desde a criação de datasets vasculares compactos até a análise de artérias, e incluem também o diagnóstico automático, a rotulação de vasos, a detecção de vasos, entre outras.

Descrevemos a seguir algumas pesquisas específicas que podem ser desenvolvidas a partir deste trabalho.

Os símbolos da representação podem ser melhorados de maneira que sejam possibilitados não apenas os registros das bordas exteriores do vaso, mas também os do raio do lúmen ao longo de cada segmento.

Podem ser incluídos novos símbolos e/ou parâmetros para conseguir representar segmentos com formas transversais não circulares. Isto, já que vasos com doenças como a estenose 
raramente têm circunferências uniformes [Rubin e Rofsky, 2012, p. 450-451].

Nossa cadeia de texto reduz drasticamente o espaço requerido para o armazenamento, porém, ele pode ser ainda mais comprimida, dividindo-se a representação textual em várias outras regras, podendo se associar cada uma delas a um determinado padrão de ramificação dentro da rede vascular. Isto impediria que repetíssemos sequências de instruções similares na representação.

Neste trabalho fazemos uso de uma representação textual, que é posteriormente transformada em um grafo, o qual faz possível a quantificação. Porém, até o momento, não foi usada a direção do fluxo sanguíneo, a qual poderia ser calculada usando métodos como os propostos na solução do problema de fluxo máximo [Kelner et al., 2014].

Neste trabalho, usamos imagens de angio-RM e angio-CT como dados de entrada, porém, nossa metodologia é independente da forma de aquisição da imagem, e requer apenas resultados de segmentação. Por esta razão, a metodologia pode ser estendida para o uso em outras modalidades, ou até em diferentes estruturas desde que estas sejam formadas por conjuntos de objetos tubulares, tais como neurônios ou encanamentos. 


\section{Referências Bibliográficas}

Ajam et al.(2017) Azimah Ajam, Azrina Abd Aziz, Vijanth Sagayan Asirvadam, Sobri Muda, Ibrahima Faye e S Jamal Safdar Gardezi. A review on segmentation and modeling of cerebral vasculature for surgical planning. IEEE Access. Citado na pág. 23

Alastruey et al.(2007) J Alastruey, KH Parker, J Peiró, SM Byrd e SJ Sherwin. Modelling the circle of willis to assess the effects of anatomical variations and occlusions on cerebral flows. Journal of biomechanics, 40(8):1794-1805. Citado na pág. 3

Bankman(2008) Isaac Bankman. Handbook of medical image processing and analysis. academic press. Citado na pág. 18

Biesdorf et al.(2009) Andreas Biesdorf, S Worz, Hendrik von Tengg-Kobligk e Karl Rohr. Automatic detection of supraaortic branches and model-based segmentation of the aortic arch from 3d cta images. Em 2009 IEEE International Symposium on Biomedical Imaging: From Nano to Macro, páginas 486-489. IEEE. Citado na pág. 13

Biesdorf et al.(2012) Andreas Biesdorf, Karl Rohr, Duan Feng, Hendrik von Tengg-Kobligk, Fabian Rengier, Dittmar Böckler, Hans-Ulrich Kauczor e Stefan Wörz. Segmentation and quantification of the aortic arch using joint $3 \mathrm{~d}$ model-based segmentation and elastic image registration. Medical image analysis, 16(6):1187-1201. Citado na pág. 12

Bullitt et al.(2003) E. Bullitt, G. Gerig, S.M. Pizer, W. Lin e S.R. Aylward. Measuring tortuosity of the intracerebral vasculature from MRA images. Medical Imaging, IEEE Transactions on, 22(9):1163-1171. Citado na pág. 4, 26, 52, 53

Bullitt et al.(2005) Elizabeth Bullitt, Donglin Zeng, Guido Gerig, Stephen Aylward, Sarang Joshi, J Keith Smith, Weili Lin e Matthew G Ewend. Vessel tortuosity and brain tumor malignancy: a blinded study1. Academic radiology, 12(10):1232-1240. Citado na pág. 26

Cao et al.(2017) Yong Cao, Yuan Zhou, Shuangfei Ni, Tianding Wu, Ping Li, Shenghui Liao, Jianzhong Hu e Hongbin Lu. Three dimensional quantification of microarchitecture and vessel regeneration by synchrotron radiation microcomputed tomography in a rat model of spinal cord injury. Journal of neurotrauma, 34(6):1187-1199. Citado na pág. 12

Centers for Disease Control and Prevention(2015) Centers for Disease Control and Prevention. Deaths: final data for 2013. National vital statistics reports: from the Centers for Disease 
Control and Prevention, National Center for Health Statistics, National Vital Statistics System, 64(2). Citado na pág. 1

Cook e Holder(2006) Diane J Cook e Lawrence B Holder. Mining graph data. John Wiley \& Sons. Citado na pág. 57

Cuntz et al.(2007) Hermann Cuntz, Alexander Borst e Idan Segev. Optimization principles of dendritic structure. Theoretical Biology and Medical Modelling, 4(1):1. Citado na pág. 14

Danielsson(1980) Per-Erik Danielsson. Euclidean distance mapping. Computer Graphics and image processing, 14(3):227-248. Citado na pág. 32

De-La-Cruz(2015) Leandro T. De-La-Cruz. Quantificação de angiogênese em imagens de membranas corioalantóicas de embrião de galinha. Dissertação de Mestrado, Matemathics and statistics Institute, University of São Paulo, São Paulo-Brasil. Citado na pág. 2, 4

Dehmeshki et al.(2014) Jamshid Dehmeshki, Adina Ion, Tim J Ellis, Francesco Doenz, AnneMarie Jouannic e Salah Qanadli. Computer aided detection and measurement of peripheral artery disease. Studies in health technology and informatics, 205:1153-1157. Citado na pág. 12

Deutsch(1996) L Peter Deutsch. Deflate compressed data format specification version 1.3. Citado na pág. 70

Duan et al.(2013) Jinghao Duan, Chunhong Hu, Shuqian Luo, Xinyan Zhao e Tailing Wang. Microcomputed tomography with diffraction-enhanced imaging for morphologic characterization and quantitative evaluation of microvessel of hepatic fibrosis in rats. PloS one, 8(10): e78176. Citado na pág. 12,15

Eleid et al.(2014) Mackram F Eleid, Raviteja R Guddeti, Marysia S Tweet, Amir Lerman, Mandeep Singh, Patricia J Best, Terri J Vrtiska, Megha Prasad, Charanjit S Rihal, Sharonne N Hayes et al. Coronary artery tortuosity in spontaneous coronary artery dissection angiographic characteristics and clinical implications. Circulation: Cardiovascular Interventions, 7(5):656-662. Citado na pág. 6

Ellwein et al.(2016) Laura Ellwein, David S Marks, Raymond Q Migrino, W Dennis Foley, Sara Sherman e John F LaDisa. Image-based quantification of 3d morphology for bifurcations in the left coronary artery: Application to stent design. Catheterization and Cardiovascular Interventions, 87(7):1244-1255. Citado na pág. 12

Frangi et al.(1998) Alejandro F Frangi, Wiro J Niessen, Koen L Vincken e Max A Viergever. Multiscale vessel enhancement filtering. Em International Conference on Medical Image Computing and Computer-Assisted Intervention, páginas 130-137. Springer. Citado na pág. 18, 19,30

Ghatwary et al.(2012) Tamer Ghatwary, Alan Karthikesalingam, Benjamin Patterson, Robert Hinchliffe, Robert Morgan, Ian Loftus, Ayman Salem, Matt M Thompson e Peter J Holt. St 
george's vascular institute protocol: an accurate and reproducible methodology to enable comprehensive characterization of infrarenal abdominal aortic aneurysm morphology in clinical and research applications. Journal of Endovascular Therapy, 19(3):400-414. Citado na pág. 12

Hamarneh e Jassi(2010) G. Hamarneh e P. Jassi. Vascusynth: Simulating vascular trees for generating volumetric image data with ground-truth segmentation and tree analysis. Computerized Medical Imaging and Graphics, 34(8):605-616. Citado na pág. 20

Heinzer et al.(2008) Stefan Heinzer, Gisela Kuhn, Thomas Krucker, Eric Meyer, Alexandra Ulmann-Schuler, Marco Stampanoni, Max Gassmann, Hugo H Marti, Ralph M"uller e Johannes Vogel. Novel three-dimensional analysis tool for vascular trees indicates complete micronetworks, not single capillaries, as the angiogenic endpoint in mice overexpressing human vegf 165 in the brain. Neuroimage, 39(4):1549-1558. Citado na pág. 11, 13

Hong e Wang(2013) Qingqi Hong e Beizhan Wang. Segmentation of vessel images using a localized hybrid level-set method. Em Image and Signal Processing (CISP), 2013 6th International Congress on, volume 2, páginas 631-635. doi: 10.1109/CISP.2013.6745243. Citado na pág. 3,18

Horsfield(1980) Keith Horsfield. Are diameter, length and branching ratios meaningful in the lung? Journal of theoretical biology, 87(4):773-784. Citado na pág. 28

Hu et al.(2011) Shuangwei Hu, Martin Lundgren e Antti J Niemi. Discrete frenet frame, inflection point solitons, and curve visualization with applications to folded proteins. Physical Review E, 83(6):061908. Citado na pág. 26

Huang et al.(2006) Su Huang, Rafail Baimouratov, Ihar Volkau e Wieslaw L Nowinski. Ivme: A tool for editing, manipulation, quantification, and labeling of cerebrovascular models. Computerized Medical Imaging and Graphics, 30(3):187-195. Citado na pág. 13

Kang et al.(2009) Dong-Goo Kang, Dae Chul Suh e Jong Beom Ra. Three-dimensional blood vessel quantification via centerline deformation. Medical Imaging, IEEE Transactions on, 28 (3):405-414. Citado na pág. 2, 4, 13

Keele(2007) Staffs Keele. Guidelines for performing systematic literature reviews in software engineering. Citado na pág. 7

Kelner et al.(2014) Jonathan A Kelner, Yin Tat Lee, Lorenzo Orecchia e Aaron Sidford. An almost-linear-time algorithm for approximate max flow in undirected graphs, and its multicommodity generalizations. Em Proceedings of the Twenty-Fifth Annual ACM-SIAM Symposium on Discrete Algorithms, páginas 217-226. SIAM. Citado na pág. 86

Kitchenham(2004) Barbara Kitchenham. Procedures for performing systematic literature reviews. Citado na pág. 7

Kocher et al.(2011) Keith E Kocher, William J Meurer, Reza Fazel, Phillip A Scott, Harlan M Krumholz e Brahmajee K Nallamothu. National trends in use of computed tomography in the emergency department. Annals of emergency medicine, 58(5):452-462. Citado na pág. 2 
Kókai et al.(1999) G. Kókai, Z. Tóth e R. Ványi. Modelling blood vessels of the eye with parametric L-systems using evolutionary algorithms. Artificial Intelligence in Medicine, páginas 433-442. Citado na pág. 21

Kuhn(1955) Harold W Kuhn. The hungarian method for the assignment problem. Naval Research Logistics (NRL), 2(1-2):83-97. Citado na pág. 59

Lee et al.(1994) Ta-Chih Lee, Rangasami L Kashyap e Chong-Nam Chu. Building skeleton models via 3-d medial surface axis thinning algorithms. CVGIP: Graphical Models and Image Processing, 56(6):462-478. Citado na pág. 32

Lesage et al.(2009) David Lesage, Elsa D Angelini, Isabelle Bloch e Gareth Funka-Lea. A review of $3 \mathrm{~d}$ vessel lumen segmentation techniques: Models, features and extraction schemes. Medical image analysis, 13(6):819-845. Citado na pág. 3, 18

Lézoray e Grady(2012) Olivier Lézoray e Leo Grady. Image processing and analysis with graphs: theory and practice. CRC Press. Citado na pág. 58

Liberati et al.(2009) Alessandro Liberati, Douglas G Altman, Jennifer Tetzlaff, Cynthia Mulrow, Peter C Gøtzsche, John PA Ioannidis, Mike Clarke, Pl J Devereaux, Jos Kleijnen e David Moher. The prisma statement for reporting systematic reviews and meta-analyses of studies that evaluate health care interventions: explanation and elaboration. PLoS medicine, 6(7): e1000100. Citado na pág. 9, 10

Lu et al.(2017) Siyu Lu, Hui Huang, Ping Liang, Gang Chen e Liang Xiao. Hepatic vessel segmentation using variational level set combined with non-local robust statistics. Magnetic resonance imaging, 36:180-186. Citado na pág. 18

Mansur e Favarato(2012) Antonio de Padua Mansur e Desidério Favarato. Mortality due to cardiovascular diseases in brazil and in the metropolitan region of são paulo: a 2011 update. Arq. bras. cardiol, 99(2):755-761. Citado na pág. 1

Marxen et al.(2009) M Marxen, JG Sled e RM Henkelman. Volume ordering for analysis and modeling of vascular systems. Annals of biomedical engineering, 37(3):542-551. Citado na pág. 13

Mazur et al.(2015) Alicja Mazur, Joanna Płaskonka e Mirela Kaczmarek. Following 3d paths by a manipulator. Archives of Control Sciences, 25(1):117-133. Citado na pág. 26

Megalooikonomou et al.(2009) Vasilis Megalooikonomou, Michael Barnathan, Despina Kontos, Predrag R Bakic e Andrew DA Maidment. A representation and classification scheme for tree-like structures in medical images: analyzing the branching pattern of ductal trees in x-ray galactograms. Medical Imaging, IEEE Transactions on, 28(4):487-493. Citado na pág. 3

Moritani et al.(2001) Toshio Moritani, David A Shrier, Yuji Numaguchi, Chihiro Takahashi, Takanori Yano, Kanji Nakai, Jianhui Zhong, Henry Z Wang, Dean K Shibata e Susan M Naselli. Diffusion-weighted echo-planar $\mathrm{mr}$ imaging of cns involvement in systemic lupus erythematosus. Academic radiology, 8(8):741-753. Citado na pág. 26 
Neugebauer et al.(2013) Mathias Neugebauer, Kai Lawonn, Oliver Beuing e Bernhard Preim. Automatic generation of anatomic characteristics from cerebral aneurysm surface models. International journal of computer assisted radiology and surgery, 8(2):279-289. Citado na pág. 12

Newman et al.(1997) WI Newman, DL Turcotte e AM Gabrielov. Fractal trees with side branching. Fractals, 5(04):603-614. Citado na pág. 28

Nowinski et al.(2009) Wieslaw L Nowinski, Ihar Volkau, Yevgen Marchenko, A Thirunavuukarasuu, Ting Ting Ng e Val M Runge. A $3 \mathrm{~d}$ model of human cerebrovasculature derived from 3t magnetic resonance angiography. Neuroinformatics, 7(1):23-36. Citado na pág. 3

Organization et al.(2014) World Health Organization et al. Global health estimates: Deaths by cause, age, sex and country, 2000-2012. Geneva, WHO, 9. Citado na pág. 1

O'Flynn et al.(2007) Padraig M O'Flynn, Gerard O'Sullivan e Abhay S Pandit. Methods for three-dimensional geometric characterization of the arterial vasculature. Annals of biomedical engineering, 35(8):1368-1381. Citado na pág. 13

Passat et al.(2006) Nicolas Passat, Christian Ronse, Joseph Baruthio, J-P Armspach e Claude Maillot. Magnetic resonance angiography: from anatomical knowledge modeling to vessel segmentation. Medical Image Analysis, 10(2):259-274. Citado na pág. 3

Perez et al.(2002) MEM Perez, AD Highes, Alice V Stanton, Simon A Thorn, Neil Chapman, Anil Anthony Bharath e Kim H Parker. Retinal vascular tree morphology: a semi-automatic quantification. Biomedical Engineering, IEEE Transactions on, 49(8):912-917. Citado na pág. 2, 4

Piccinelli et al.(2009) Marina Piccinelli, Alessandro Veneziani, David Steinman, Andrea Remuzzi, Luca Antiga et al. A framework for geometric analysis of vascular structures: application to cerebral aneurysms. Medical Imaging, IEEE Transactions on, 28(8):1141-1155. Citado na pág. 13

Piccinelli et al.(2012) Marina Piccinelli, David A Steinman, Yiemeng Hoi, Frank Tong, Alessandro Veneziani e Luca Antiga. Automatic neck plane detection and 3d geometric characterization of aneurysmal sacs. Annals of biomedical engineering, 40(10):2188-2211. Citado na pág. 12

Pratt et al.(2017) Harry Pratt, Bryan M Williams, Jae Ku, Frans Coenen e Yalin Zheng. Automatic detection and identification of retinal vessel junctions in colour fundus photography. Em Annual Conference on Medical Image Understanding and Analysis, páginas 27-37. Springer. Citado na pág. 18

Prezemyslaw e Lindenmayer(1996) P. Prezemyslaw e A. Lindenmayer. The algorithmic beauty of plants, 1996. Citado na pág. 21 
Rahaghi et al.(2016) FN Rahaghi, JC Ross, M Agarwal, G González, CE Come, AA Diaz, G Vegas-Sánchez-Ferrero, A Hunsaker, R San José Estépar, AB Waxman et al. Pulmonary vascular morphology as an imaging biomarker in chronic thromboembolic pulmonary hypertension. Pulmonary circulation, 6(1):70. Citado na pág. 12

Rahman et al.(2008) Mohammed Riazur Rahman, M Ameer Ali e Golam Sorwar. Finding significant points for parametric curve generation techniques. IETECH Journal of Advanced Computations, 2(2):107. Citado na pág. 40, 41

Rajna et al.(2015) Zalán Rajna, Anja Keskinarkaus, Vesa Kiviniemi e Tapio Seppänen. Speeding up the file access of large compressed nifti neuroimaging data. Em Engineering in Medicine and Biology Society (EMBC), 2015 37th Annual International Conference of the IEEE, páginas 654-657. IEEE. Citado na pág. 70

Rozenberg(1980) Grzegorz Rozenberg. The Mathematical Theory of L-Systems. Academic Press, primeira edição. Citado na pág. 21

Rubin e Rofsky(2012) Geoffrey D Rubin e Neil M Rofsky. CT and MR angiography: comprehensive vascular assessment. Lippincott Williams \& Wilkins. Citado na pág. 17, 86

Saha et al.(2016) Punam K Saha, Gunilla Borgefors e Gabriella Sanniti di Baja. A survey on skeletonization algorithms and their applications. Pattern Recognition Letters, 76:3-12. Citado na pág. 20

Sato et al.(1997) Yoshinobu Sato, Shin Nakajima, Hideki Atsumi, Thomas Koller, Guido Gerig, Shigeyuki Yoshida e Ron Kikinis. 3d multi-scale line filter for segmentation and visualization of curvilinear structures in medical images. Em CVRMed-MRCAS'97, páginas 213-222. Springer. Citado na pág. 19, 20, 30

Sato et al.(1998) Yoshinobu Sato, Shin Nakajima, Nobuyuki Shiraga, Hideki Atsumi, Shigeyuki Yoshida, Thomas Koller, Guido Gerig e Ron Kikinis. Three-dimensional multi-scale line filter for segmentation and visualization of curvilinear structures in medical images. Medical image analysis, 2(2):143-168. Citado na pág. 19, 30

Scorcioni et al.(2008) Ruggero Scorcioni, Sridevi Polavaram e Giorgio A Ascoli. L-measure: a web-accessible tool for the analysis, comparison and search of digital reconstructions of neuronal morphologies. Nature protocols, 3(5):866-876. Citado na pág. 14

Scott e Luo(2001) Ethan K Scott e Liqun Luo. How do dendrites take their shape? Nature neuroscience, 4(4):359-365. Citado na pág. 14

Soille(2013) Pierre Soille. Morphological image analysis: principles and applications. Springer Science \& Business Media. Citado na pág. 31, 32

Strahler(1957) Arthur N Strahler. Quantitative analysis of watershed geomorphology. Eos, Transactions American Geophysical Union, 38(6):913-920. Citado na pág. 28 
Suetens(2009) Paul Suetens. Fundamentals of medical imaging. Cambridge university press. Citado na pág. 1,2

Sun e Cao(2011) Zhonghua Sun e Yan Cao. Multislice ct angiography assessment of left coronary artery: correlation between bifurcation angle and dimensions and development of coronary artery disease. European journal of radiology, 79(2):e90-e95. Citado na pág. 4

Szczerba e Székely(2002) D. Szczerba e G. Székely. Macroscopic modeling of vascular systems. Medical Image Computing and Computer-Assisted Intervention-MICCAI 2002, páginas 284-292. Citado na pág. 20

Tokunaga(1978) Eiji Tokunaga. Consideration on the composition of drainage networks and their evolution. Citado na pág. 28

Valverde(2012) Miguel A. G. Valverde. Three-dimensional synthetic blood vessels generation using stochastic Lindenmayer systems. Dissertação de Mestrado, Matemathics and statistics Institute, University of Sao Paulo, São Paulo-Brasil. Citado na pág. 3, 20, 21, 22, 23, 31, 43, 49, 57,65

Valverde et al.(2013) Miguel A. G. Valverde, Maysa M. G. Macedo, Choukri Mekkaoui e Macel P. Jackowski. Three-dimensional synthetic blood vessel generation using stochastic Lsystems. SPIE Medical Imaging 2013. Citado na pág. 21, 43, 44, 49, 61

Valverde et al.(2015) Miguel A G Valverde, Jihan M Zoghbi, Fabricio Pereira, Jean-Paul Beregi, Choukri Mekkaoui e Marcel P Jackowski. Characterization of vascular tree architecture using the tokunaga taxonomy. Em SPIE Medical Imaging, páginas 941438-941438. International Society for Optics and Photonics. Citado na pág. 4, 12

Van Bemmel et al.(2004) C. M. Van Bemmel, M. A. Viergever e W. J. Niessen. Semiautomatic segmentation and stenosis quantification of $3 \mathrm{~d}$ contrast-enhanced $\mathrm{mr}$ angiograms of the internal carotid artery. Magnetic Resonance in Medicine, 51(4):753-760. Citado na pág. 3, 4

Versteylen et al.(2013) Mathijs O Versteylen, Bas L Kietselaer, Pieter C Dagnelie, Ivo A Joosen, Admir Dedic, Rolf H Raaijmakers, Joachim E Wildberger, Koen Nieman, Harry J Crijns, Wiro J Niessen et al. Additive value of semiautomated quantification of coronary artery disease using cardiac computed tomographic angiography to predict future acute coronary syndrome. Journal of the American College of Cardiology, 61(22):2296-2305. Citado na pág. 2

Wang et al.(2004) Zhou Wang, Alan C Bovik, Hamid R Sheikh e Eero P Simoncelli. Image quality assessment: from error visibility to structural similarity. IEEE transactions on image processing, 13(4):600-612. Citado na pág. 68

Washington et al.(2011) Christopher B Washington, Judy Shum, Satish C Muluk e Ender A Finol. The association of wall mechanics and morphology: a case study of abdominal aortic aneurysm growth. Journal of biomechanical engineering, 133(10):104501. Citado na pág. 13, 14,15 
Wolterink et al.(2016) Jelmer M Wolterink, Tim Leiner, Bob D de Vos, Robbert W van Hamersvelt, Max A Viergever e Ivana Išgum. Automatic coronary artery calcium scoring in cardiac ct angiography using paired convolutional neural networks. Medical image analysis. Citado na pág. 12

Worz et al.(2010) Stefan Worz, Hendrik von Tengg-Kobligk, Verena Henninger, Fabian Rengier, Hardy Schumacher, Dittmar Bockler, Hans-Ulrich Kauczor e Karl Rohr. 3-d quantification of the aortic arch morphology in 3-d cta data for endovascular aortic repair. IEEE Transactions on Biomedical Engineering, 57(10):2359-2368. Citado na pág. 13, 14

Wright et al.(2013) Susan N Wright, Peter Kochunov, Fernando Mut, Maurizio Bergamino, Kerry M Brown, John C Mazziotta, Arthur W Toga, Juan R Cebral e Giorgio A Ascoli. Digital reconstruction and morphometric analysis of human brain arterial vasculature from magnetic resonance angiography. Neuroimage, 82:170-181. Citado na pág. 12, 14, 61, 65

Zaliapin e Kovchegov(2012) Ilia Zaliapin e Yevgeniy Kovchegov. Tokunaga and horton selfsimilarity for level set trees of markov chains. Chaos, Solitons \& Fractals, 45(3):358-372. Citado na pág. 28

Zeide e Gresham(1991) Boris Zeide e Charles A Gresham. Fractal dimensions of tree crowns in three loblolly pine plantations of coastal south carolina. Canadian Journal of Forest Research, 21(8):1208-1212. Citado na pág. 28

Zhou et al.(2011) Mi Zhou, Jun Zhao e Falei Yuan. Quantitative analysis of vessel morphology using micro-computed tomography. Em 2011 4th International Conference on Biomedical Engineering and Informatics (BMEI), volume 1, páginas 457-461. IEEE. Citado na pág. 13, 14

Zhu e Chung(2013) Ning Zhu e Albert Chung. Optimal and efficient segmentation for $3 \mathrm{~d}$ vascular forest structure with graph cuts. Em Image Processing (ICIP), 2013 20th IEEE International Conference on, páginas 1135-1139. IEEE. Citado na pág. 3, 18

Zoghbi et al.(2014) Jihan M Zoghbi, Leandro T De La Cruz, Miguel A G Valverde, Marcel P Jackowski, J C F Vieira, A M A Liberatore e Ivan H J Koh. Graph based characterization of microcirculation in sepsis using sidestream dark field imaging. Em 2014 27th SIBGRAPI Conference on Graphics, Patterns and Images (SIBGRAPI), páginas 312-318. IEEE. Citado na pág. 28,55 